\title{
DEVELOPING A MODEL OF POST-WORKSHOP CONSULTATION FOR CLINICIANS \\ LEARNING TO DELIVER COGNITIVE PROCESSING THERAPY FOR POSTTRAUMATIC STRESS DISORDER
}

\author{
by \\ Meredith Sara Herman Landy \\ Bachelor of Arts, McGill University, 2008 \\ Master of Arts, New York University, 2010
}

A dissertation
presented to Ryerson University

in partial fulfillment of the

requirements for the degree of

Doctor of Philosophy

in the program of

Psychology

Toronto, Ontario, Canada, 2017

CMeredith Landy 2017 


\begin{abstract}
Author's Declaration
I hereby declare that I am the sole author of this dissertation. This is a true copy of the dissertation, including any required final revisions, as accepted by my examiners.

I authorize Ryerson University to lend this dissertation to other institutions or individuals for the purpose of scholarly research.

I further authorize Ryerson University to reproduce this dissertation by photocopying or by other means, in total or in part, at the request of other individuals for the purpose of scholarly research.
\end{abstract}

I understand that my dissertation may be made electronically available to the public. 


\begin{abstract}
Developing a model of post-workshop consultation for clinicians learning to deliver Cognitive Processing Therapy for posttraumatic stress disorder
\end{abstract}

Doctor of Philosophy, 2017

Meredith Sara Herman Landy

Psychology

Ryerson University

Best practice in training clinicians to deliver evidence-based psychotherapies includes workshop attendance followed by post-workshop consultation. Although previous research highlights the importance of consultation, little is known about what makes for effective consultation, and no model of clinical consultation currently exists. The primary aims of this study were to identify the primary elements of consultation, and develop a model of consultation in a sample of clinicians learning to deliver Cognitive Processing Therapy (CPT; Resick, Monson, \& Chard, 2014), an evidence-based psychotherapy for posttraumatic stress disorder. The study was conducted from a realist perspective, a paradigm that is particularly useful for theory building. The study involved the participation of mental health clinicians $(N=41)$ who attended one of five CPT workshops, and CPT consultants ( $N$ =6) who provided the clinicians with post-workshop consultation for six months following the workshop. Thirty audio recorded consultation calls were randomly selected and transcribed. The data was coded and analyzed using thematic analysis. The kappa statistic measuring inter-rater reliability was .80. The following 
contextual factors were identified: access to group support, clinicians joining late, clinicians not having content to discuss, study participation, and technological disruptions. Knowledge consolidation and case conceptualization were identified as the overarching functions of consultation. The remaining elements of consultation were classified into the following themes: Organization, Asking for Help, Directive Instruction, Non-Directive Instruction, and Provision of Feedback. Two hypothesized mechanisms of consultation, reflection and connectedness, were identified. A comprehensive model positing how consultation works was presented. Additionally, three sets of context-mechanism-outcome configurations were presented. The model of consultation was compared to the model of clinical supervision proposed by Milne and colleagues (2008). Finally, the usefulness of Kolb’s (1984) model of experiential learning was explored as a framework for understanding the learning that occurs during clinical consultation. Based on the study's findings, several recommendations for clinical practice were made. An important next step is to test the proposed theory, and to assess the relationship between the use of various elements of consultation and the development of proficiency in delivering CPT. 


\section{Acknowledgements}

Thank you to my supervisor, Dr. Candice M. Monson for your unwavering support throughout graduate school. Thank you for teaching me to celebrate the success of process rather than outcome, and for modeling the value in adopting a flexible perspective.

Thank you, Dr. Kelly E. McShane for your help conceptualizing, navigating, and executing this project. I am grateful for the challenging and thought provoking conversations we have had, and for the ways they have shaped my thinking.

Thank you, Sheena Bance and Esztella Vezer for the many hours you dedicated to coding and analyzing this data.

Thank you, Dr. Karen Milligan, Dr. Elizabeth McCay, and Dr. Martin Drapeau for your willingness to contribute your time.

Thank you to my husband, Dr. Joshua S. Landy for your love, encouragement, and humour. Thank you for the hundreds, perhaps thousands, of meals you have cooked for me that sustained me throughout graduate school. 


\section{Dedication}

This dissertation is dedicated in loving memory of my father-in-law, Keith M. Landy, who applied his professional acumen in pursuit of human rights and social justice. 


\section{Table of Contents}

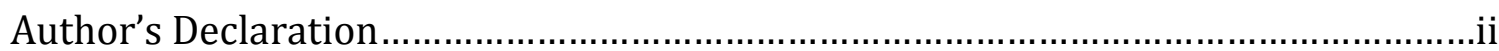

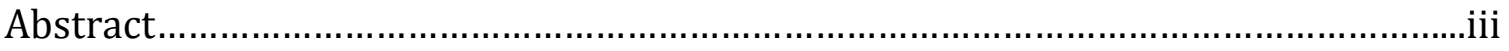

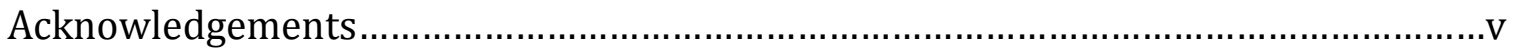

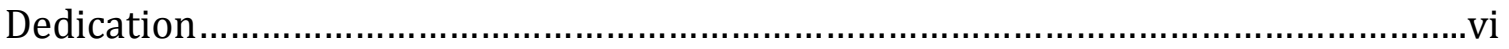

Table of Contents....................................................................................................

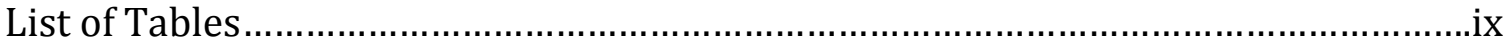

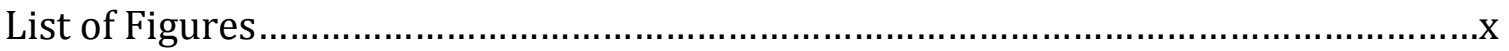

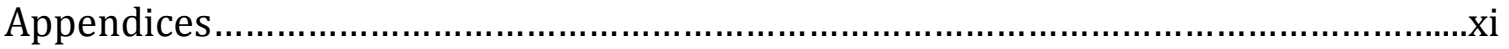

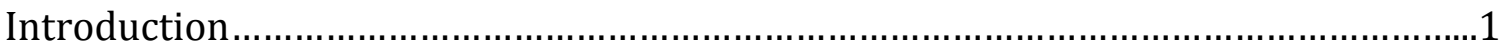

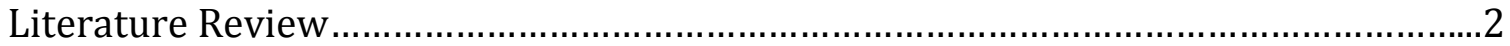

Dissemination of Evidence-Based Psychotherapies........................................2

The Importance of Post-Workshop Support...................................................

Differentiating between Supervision and Consultation..................................6

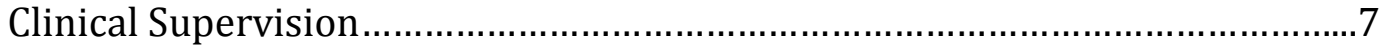

A Model of Clinical Supervision...................................................................10

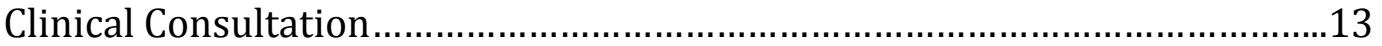

Group Delivery of Consultation.................................................................

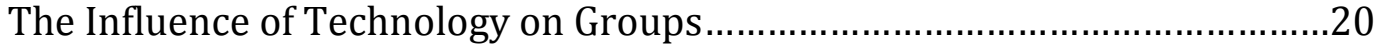

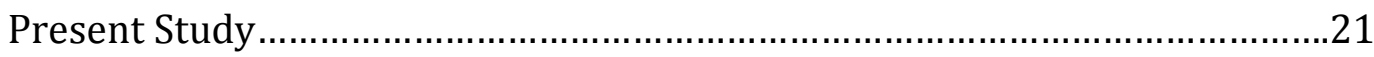

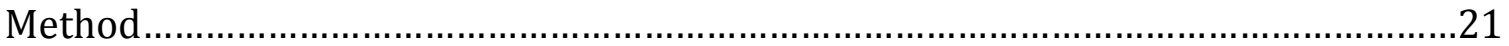

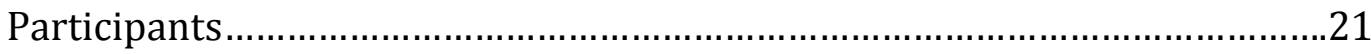

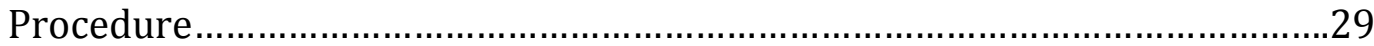




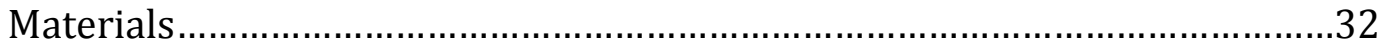

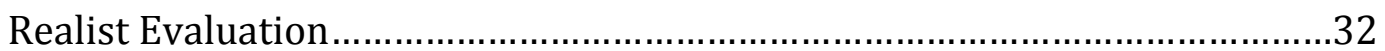

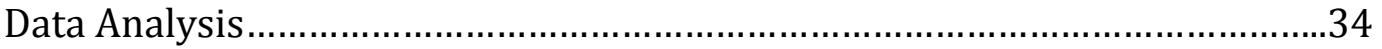

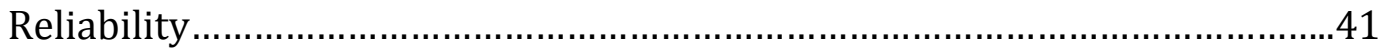

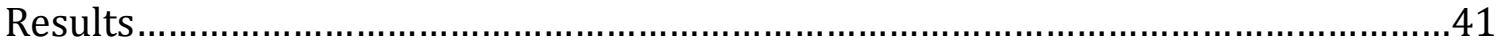

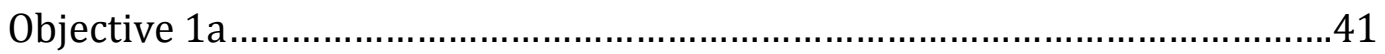

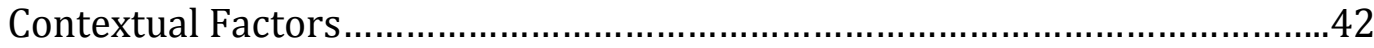

Primary Elements of Consultation........................................................50

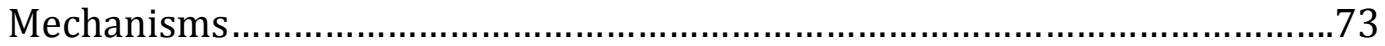

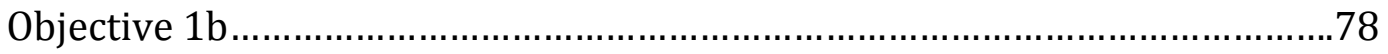

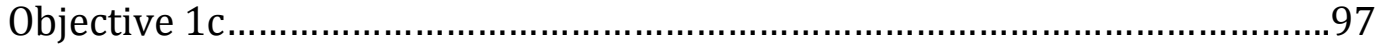

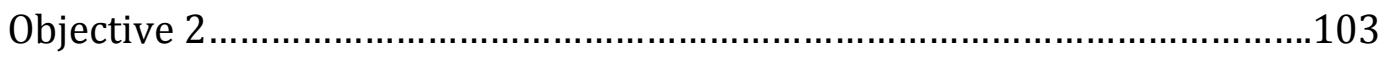

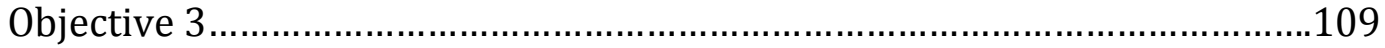

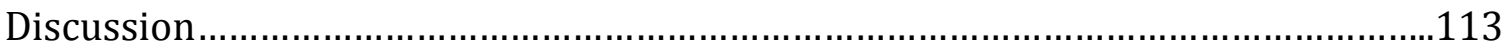

Recommendations for Clinical Practice.......................................................118

Limitations and Future Directions....................................................122

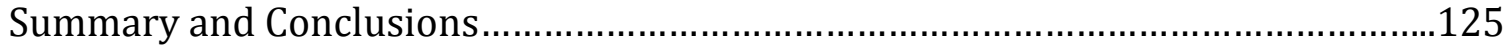

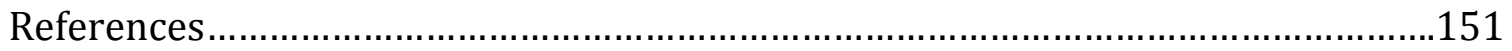




\section{List of Tables}

Table 1: Clinician Demographics..................................................................22

Table 2: Clinician Psychotherapy and Training Experience...........................25

Table 3: Consultant Demographics.........................................................28 


\section{List of Figures}

Figure 1: Model of Clinical Supervision ......................................................11

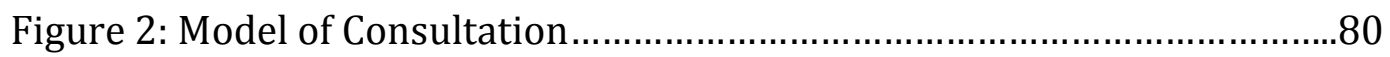

Figure 3: Context-Mechanism-Outcome Configuration 1................................98

Figure 4: Context-Mechanism-Outcome Configuration 2.............................100

Figure 5: Context-Mechanism-Outcome Configuration 3.............................102

Figure 6: Complete Context-Mechanism-Outcome Configuration..................104 


\section{List of Appendices}

Appendix A: Comparison of Three Post-Workshop Conditions................................128

Appendix B: Codebook: Contextual Factors.........................................................129

Appendix C: Codebook: Primary Elements of Consultation ....................................131 


\section{Introduction}

Policymakers are investing billions of dollars to train clinicians to deliver evidence-based psychotherapies (EBPs; Karlin et al., 2010; McHugh \& Barlow, 2010). Attending an EBP workshop often leads to increased provider knowledge (Fixsen, Naoom, Base, Friedman, \& Wallace, 2005), but is insufficient for the majority of clinicians to become proficient in delivering the intervention with fidelity (Beidas \& Kendall, 2010; Miller, Yahne, Moyers, Martinez, \& Pirritano, 2004; Sholomskas et al., 2005). Fidelity to treatment is indispensable, as it is a strong predictor of symptom change and clinical outcome in the treatment of a variety of mental health conditions (e.g. Martino, Ball, Nich, Frankforter, \& Carroll, 2008; Schoenwald, Carter, Chapman, \& Sheidow, 2008; Strunk, Brotman, DeRubeis, \& Hollon, 2010). Providing clinicians with post-workshop support, such as group consultation with an expert, improves their fidelity to treatment (Beidas, Edmunds, Marcus, \& Kendall, 2012; Schoenwald, Sheidow, \& Letournea, 2004).

In spite of data suggesting that consultation is important (Beidas et al., 2013; Beidas, Edmunds, Marcus, \& Kendall, 2012; Edmunds, Beidas, \& Kendall, 2013), little is known about the specifics of consultation and what are the necessary components that contribute to successful consultation (Milne \& Westerman, 2001; Beidas et al., 2013; Nadeem et al., 2013b; Rakovshik \& McManus, 2010). An important next step is delineating the primary elements of clinical consultation (Beidas \& Kendall, 2010; Herschell, Kolko, Baumann, \& Davis, 2010; Rakovshik \& McManus, 2010; Weisz, Ugueto, Herren, Afienko, \& Rutt, 2011), including defining and describing their function. Therefore, the aim of the present study was to develop a model of clinical 
consultation in a sample of clinicians learning to deliver Cognitive Processing Therapy (CPT; Resick, Monson, \& Chard, 2014), an evidence-based treatment for posttraumatic stress disorder (PTSD) recommended in the treatment guidelines published by the International Society for Traumatic Stress Studies (ISTSS; Foa, Keane, Friedman, \& Cohen, 2005).

Realism is a research paradigm that is particularly useful for theory building (Pawson \& Tilley, 1997). Specifically, realist evaluation emphasizes identifying an intervention's hypothesized mechanisms and the contextual factors under which it can be optimized in order to propose a theory of how the intervention works. Accordingly, this study was conducted from a realist perspective in order to identify the mechanisms through which clinical consultation functions, and the contextual factors that contribute to its success. The proposed model describing what is posited to occur during clinical consultation is a first step toward replicating that process in clinical settings, and testing which elements of consultation are most closely associated with proficient delivery of CPT.

\section{Literature Review}

\section{Dissemination of Evidence-Based Psychotherapies}

Over the past several decades, there has been a proliferation of empirically supported treatments used to treat a variety of mental health conditions and substance use problems (e.g., Dunn, Deroo, \& Rivara, 2001; Irvin, Bowers, Dunn, \& Wang, 1999; Leshner, 1999). Despite the prevalence of efficacious treatments, many patients do not receive these therapies for their condition (President's New Freedom Commission on Mental Health, 2003). This is largely because development 
of these therapies has far outpaced their implementation in real-world clinical settings (Fixsen et al., 2005; Ganju, 2003; Gotham, 2006). Previous research has identified a lack of training in the delivery of EBPs as a contributor to clinician underutilization (Kazdin, 2008; McHugh \& Barlow, 2010; Weissman et al., 2006; Williams \& Martinez, 2008). The National Institute of Mental Health recently identified research on implementation of EBPs as a high-priority item (Proctor et al., 2009). The United States Department of Veterans Affairs (VA) has undertaken a large effort to disseminate Cognitive Processing Therapy (CPT; Resick \& Schnicke, 1992) and Prolonged Exposure (PE; Foa, Hembree, \& Rothbaum, 2007), evidencebased psychotherapies for PTSD, throughout the VA health care system (Karlin et al., 2010). Veterans Affairs Canada (VAC) and its national network of Operational Stress Injury (OSI) clinics are engaged in a similar effort to disseminate evidence-based therapies for PTSD to its clinicians who treat Canadian military veterans (Shields, 2011). This study's parent study, funded by the Canadian Institute of Health Research (CIHR), represents early efforts to disseminate CPT to VAC and OSI clinicians.

To explain how treatments are transferred from research to practice settings, Gotham (2004) proposed a three-component diffusion model: development of effective treatments, dissemination, and implementation. Dissemination involves education about evidence-based treatments in such a way that clinicians and organizations can adopt them. Disseminating EBPs through workshops and didactic seminars, which are typically between a half-day to two days in duration, is the most common means of teaching these therapies to clinicians (Sholomskas et al., 2005). 
Workshops are an effective way for clinicians to acquire information and deepen their provider knowledge (Fixsen et al., 2005); however, attending a single workshop is not enough for the majority of clinicians to be able to deliver a new treatment with fidelity to protocol (Beidas \& Kendall, 2010; Miller et al., 2004; Sholomskas et al., 2005; Walters, Matson, Baer, \& Ziedonis, 2005). Three recent literature reviews on the use of education as an implementation strategy have independently emphasized the importance of offering clinicians ongoing support following training (Beidas \& Kendall, 2010; Herschell et al., 2010; Rakovshik \& McManus, 2010). For seasoned therapists, this support usually comes in the form of consultation. Research examining behaviour change among various types of healthcare providers has highlighted the integral role of consultation or coaching in this process (Grimshaw et al., 2001; Grol \& Grimshaw, 1999, 2003; Joyce \& Showers, 2002). The "gold-standard" for training clinicians in EBPs is the provision of a therapy manual, attendance at a workshop, and post-workshop support, often in the form of such consultation (Sholomskas et al., 2005).

\section{The Importance of Post-Workshop Support}

Researchers have consistently recommended providing clinicians with postworkshop support, such as access to supervision or consultation, to improve their fidelity to treatment and to promote implementation in clinical settings (Andrzejewski, Kirby, Morral, \& Iguchi, 2001; Beidas et al., 2012; Godley, Garner, Smith, Meyers, \& Godley, 2011; Rakovshik, McManus, Vazquez-Montes, Muse, \& Ougrin, 2016; Schoenwald, Sheidow, \& Chapman, 2009). It has been suggested that clinicians mostly engage in passive learning when they attend a workshop; 
therefore, supervision or consultation, which encourages active learning through interaction and reflection, is thought to be a useful complement (El-Tannir, 2002). To develop expertise, providers must deliberately and repeatedly practice new behaviours and receive corrective feedback from an instructor until the new task becomes familiar to them (McGaghie, Siddall, Mazmanian, \& Myers, 2009). Results from one study showed a dose-response effect between consultation and fidelity to protocol (Beidas et al., 2012). Specifically, in a sample of clinicians learning to deliver cognitive-behavioural therapy for youth anxiety, each additional hour of consultation improved therapist adherence by .4 and skill by .3 on a 7-point Likert scale.

A relatively new field of study has focused on how to best support clinician education in EBPs. Researchers have investigated the relative effectiveness of different therapist training strategies by varying the modality of support available to clinicians after a didactic workshop. One study looked at five different types of training (workshop only, workshop and feedback on practice tapes, workshop plus coaching, workshop plus coaching and feedback, and self-training control) with 140 substance abuse professionals who attended a Motivational Interviewing (MI) workshop (Miller et al., 2004). Researchers found that clinicians in the coaching and feedback condition became the most proficient in MI. In another study about postworkshop support modalities in clinicians from community substance abuse treatment programs learning MI, researchers found that clinicians with a graduate degree benefitted most from support that involved feedback, coaching, and discussing audio recorded counseling sessions (Carpenter et al., 2012). 
In a third study, researchers compared three methods that were used to train 78 community-based clinicians to implement a cognitive-behavioural therapy (CBT) for substance use protocol (Sholomskas et al., 2005). The three conditions were: a) exposure to the CBT manual only, b) exposure to the CBT manual plus an interactive website, or c) attendance at a 3-day didactic workshop followed by post-workshop support that involved expert review of clincians' audio recorded practice session tapes and follow-up discussion by telephone. Results showed that clinicians' ability to implement CBT was highest in the group that attended the workshop and received supervision, followed by the group that had access to the interactive website. Taken together, these studies demonstrate that clinicians benefit from receiving support after attending a workshop. Despite the importance of consultation (Beidas et al., 2013), little is known about what leads to successful consultation, and there is no model of clinical consultation delineating its primary elements (Nadeem, Gleacher, \& Beidas, 2013). To address this gap in the literature, this project aims to identify and describe the primary elements of consultation using a sample of clinicians learning to deliver CPT.

\section{Differentiating between Supervision and Consultation}

Supervision and consultation are both educational processes that aim to improve the quality of services provided to patients (Caplan \& Caplan, 1993; Milne, Aylott, Fitzpatrick, \& Ellis, 2008). Given this, models of supervision are useful as a departure point for developing a model of consultation; however, there are some notable differences between supervision and consultation. The term "supervision" is generally used to describe the guidance given by an independently licensed clinician 
who is helping a student who is not independently licensed to develop his or her clinical abilities, which can be either specific techniques or protocols, or general therapeutic skills. Though the student provides direct clinical care, the supervisor bears legal responsibility for the care provided in the case. Due to the nature of student learning, it is generally preferable for supervision to occur in an individual format. The term "consultation" is used to describe the feedback and support offered by expert clinicians to independently licensed clinicians on specific topics, techniques, or protocols. Since therapists receiving consultation are presumed to be skilled clinicians, consultation is usually narrower in focus and often occurs in groups. Since clinician learners are independent practitioners, they bear legal responsibility for their own cases (Alban \& Frankel, 2007; Caplan \& Caplan, 1993).

\section{Clinical Supervision}

Given the shared facets among consultation and supervision, there is potential for the literature on supervision to inform research on consultation. Bernard and Goodyear (1992) have defined supervision as an interpersonal relationship whereby the supervisor facilitates the development of therapeutic competence in the supervisee. Supervision is an important component of clinician learning in many healthcare professions (e.g. physicians, social workers, psychologists, nurses).

Despite the centrality of supervision in education and routine practice, the construct of supervision is poorly defined (Faugier, 1994), and has not been guided by a unified set of principles (Beidas \& Kendall, 2010). Although there have been several attempts to delineate what components of supervision are essential for 
effective training, the literature remains largely theoretical and anecdotal (Bogo \& McKnight, 2006). In reviewing the therapist training literature from 1960 to 1978, Ford (1979) tried to explain what makes for effective supervision. After careful analysis, he concluded that there was little evidence that the training methods employed at the time of writing reliably improved trainees' skill levels sufficiently for them to be considered competent treatment providers.

Although supervision continues to be regarded as an indispensable component of clinical training, the evidence base for how supervision works and what supervision ought to include is sparse (Milne \& Westerman, 2001). Researchers have found that, when supervising their own students, most supervisors draw on their own learning experience (Falender \& Shafranske, 2004). Supervisors may also apply the principles and techniques they use in therapy (e.g., Socratic dialogue) to supervision (Milne \& James, 2000; Townend, Iannetta, \& Freeston, 2002).

In a recently conducted survey, CBT therapists identified what they believed were the methods that most contributed to their learning during supervision (Bennett-Levy, McManus, Westling, \& Fennell, 2009). Results showed that clinicians found modeling/ demonstration, lectures, and reading to be the most effective in enhancing declarative knowledge, whereas enactive methods, such as role-play and self-experiential work, were perceived as most effective in enhancing procedural skills. Therapists viewed experiential work and reflective practice as most helpful for enhancing reflective ability and interpersonal skills. One of the limitations of this 
study was the inability to assess whether clinicians' perceptions of effective consultation were meaningfully connected to clinician learning or patient outcomes.

In their review of the literature on clinical supervision in training, Schoenwald, Sheidow, and Chapman (2009) noted the scarcity of studies examining the impact of supervision on client outcomes. They identified only 12 studies on this topic published between 1981 and 1997, with only 3 of them published in or after 1993. These writers also highlighted the profound methodological limitations of these studies. For example, they noted the lack of validated measures to assess supervision quality, the ambiguity regarding which components of therapy ought to be influenced by supervision, and the vague definitions and measures of client outcomes. Notably, the most frequently assessed client outcome has been satisfaction with therapy. Among the most severe methodological limitations were the prevalent use of small samples of supervisors and trainees, and the improper use of statistical analyses.

Davy (2002) wrote that the ongoing debate over the rationale, objectives, and desirable ingredients of supervision has made it difficult to generate a strong evidence base to inform the discussion. One of the biggest impediments to conducting good research on supervision and consultation is that both modalities remain poorly defined and conceptualized (Milne et al., 2008). In an effort to improve the quality and clinical utility of the supervision literature, Milne and colleagues proposed a basic model of supervision that can be tested empirically (Milne et al., 2008). 


\section{A Model of Clinical Supervision}

To address deficiencies in the clinical supervision literature, Milne and colleagues (2008) conducted a "best evidence synthesis" review of the literature to develop a model explaining how clinical supervision works (see Figure 1). Their model is grounded in empirical literature and can be tested. This approach involved creating a codebook detailing the extraction of relevant qualitative and quantitative information from the articles reviewed. After coding the articles, the reviewers conducted reliability checks to ensure consistency and reduce bias among coders. Based on this careful extraction, the reviewers organized key findings according to contextual variables, supervision interventions, and outcomes.

Contextual variables were defined as both qualitative and quantitative factors that influenced the strength of the association between the independent and dependent variables. Supervision interventions, referred to as "mediators" in this context, were defined as mechanisms that explained the association between the independent and dependent variables. For example, Socratic dialogue was conceptualized as a mediator. Outcomes were defined as the change process, or the means through which the supervision intervention influenced the outcome variable. Additionally, the resultant change in a supervisee's understanding was also treated as an outcome. For example, supervisee's improved understanding was an outcome variable, as was the process of reflection, which was thought to increase the supervisee's understanding. To construct a model of clinical supervision, the 


\section{Context}

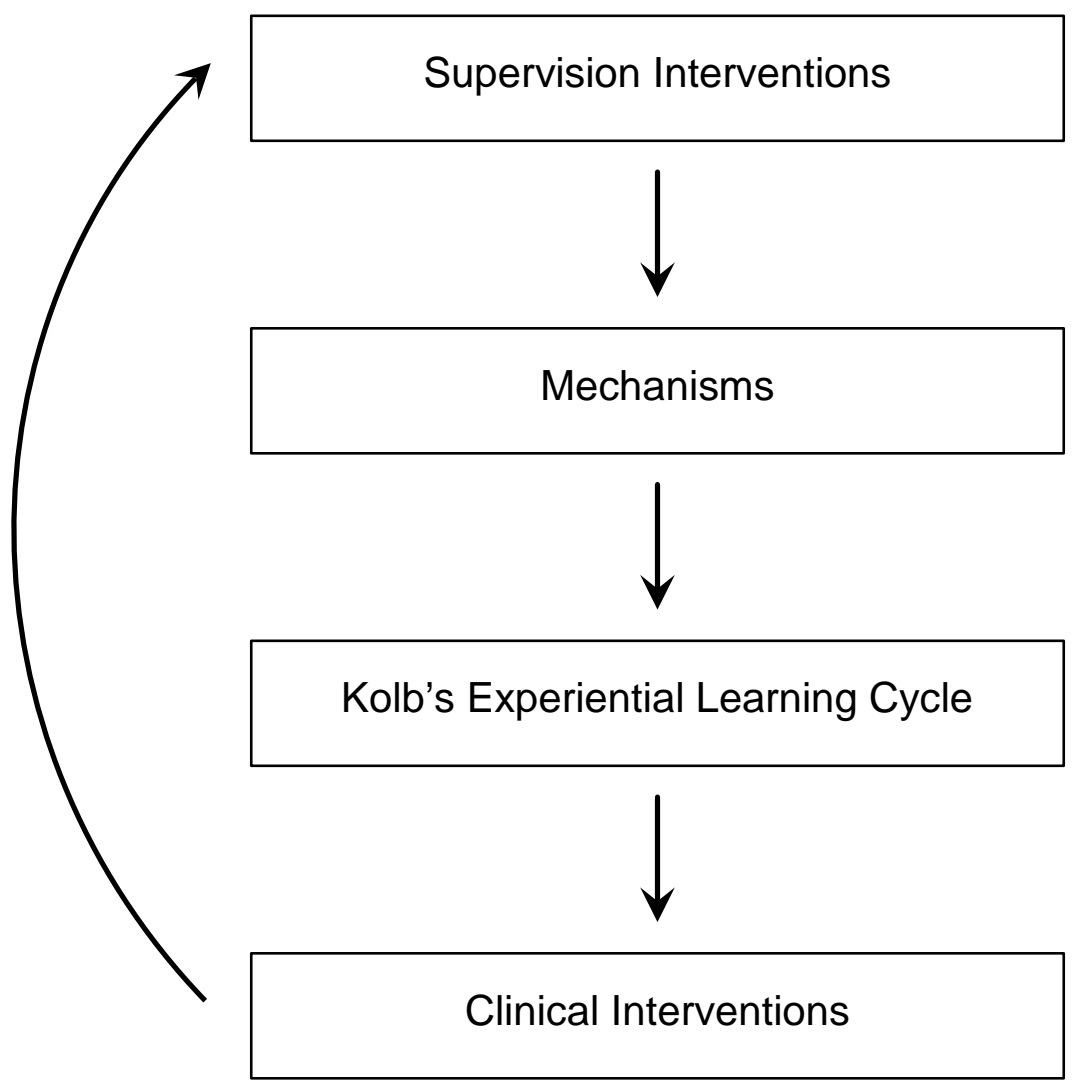

Figure 1. Model of clinical supervision. Adapted from Milne, D., Aylott, H., Fitzpatrick, H., \& Ellis, M.V. (2008). How does clinical supervision work? Using a "best evidence synthesis" approach to construct a basic model of supervision. The Clinical Supervisor, 27(2), 170-190. doi:10.1080/07325220802487915 
reviewers illustrated how contextual variables, supervision interventions, and outcomes were connected.

The researchers identified 35 contextual variables, which they collapsed into 5 themes: general organizational context, intervention factors, research factors, learning, and participants. A mean of 2.1 contextual variables were cited per study, with administrative support being the most frequently mentioned contextual variable. The reviewers indicated whether each contextual variable had a positive or negative influence on supervision. Most factors were judged to have a positive impact on supervision.

The researchers identified 26 supervision interventions that influenced the effectiveness of supervision. The most frequently cited interventions were teaching and instruction ( $75 \%$ of studies), use of corrective feedback ( $63 \%$ of studies), videobased or live observation of the supervisee ( $42 \%$ of studies), goal-setting (38\% of studies), and question-and-answer methods (38\% of studies). A mean of 5.4 supervision interventions were cited per study, suggesting that supervision typically includes the use of multiple educational techniques.

The researchers identified 28 outcome variables. Among them were changes in the supervisees' attitudes and motivation, improved skills, and increased emotional self-awareness. The writers used Kolb's (1984) experiential learning cycle as a framework to help explain how supervision interventions are posited to lead to the desired outcomes in supervisees. Kolb postulated that experiential learning occurs in four modes: experiencing, reflecting, conceptualizing, and experimenting. The reviewers found that $82 \%$ of the processes that led to the 
outcomes of interest could be classified according to Kolb's experiential learning cycle. The most frequently cited process was "experiencing," which was described in $43 \%$ of the studies. This suggests that experiential learning may be an integral component of effective supervision.

In sum, Milne and colleagues (2008) found that contextual variables facilitated a learning context, which allowed for effective supervision interventions to produce desired outcomes. Additionally, this model of supervision delineated various corrective feedback methods. In many studies, supervisees were asked to rate the relative benefit of different supervision interventions, and the results of supervisee feedback were used to inform and improve future interventions.

Milne and colleagues (2008) noted that their model is consistent with the work of previous reviewers (e.g., Gregoire, Propp, \& Poertner, 1998; Harkness \& Poertner, 1989; Milne \& James, 2000). They identified that an important next step in research is for other researchers to test their model experimentally, and to examine the specific relationships between the learning that occurs in supervision and the desired clinical outcomes. Developing a model of clinical consultation is a logical extension of Milne and colleagues' work.

\section{Clinical Consultation}

Although a comprehensive model of clinical consultation has not yet been developed, a growing body of research has begun to reveal some of the elements of effective consultation. One group of researchers addressed this topic by outlining the primary functions of consultation (Nadeem et al., 2013a). Based on a review of the literature and their experiences as consultants, they identified the following 
functions of consultation: 1) continued training (e.g., case conceptualization, further training on the primary components of the intervention); 2) problem-solving implementation barriers (e.g, at the provider, client, or community level); 3) provider engagement (e.g., leadership); 4) case support (e.g., intervention-client fit, skill application and refinement); 5) accountability (i.e., keeping consultants accountable to what they have agreed to); 6) mastery skill-building (e.g., modeling, behavioural rehearsal); 7) appropriate treatment adaptation (e.g., client age, presentation, values); and 8) planning for sustainability (i.e., helping clinicians and organizations continue to use a therapy over time).

In another study, researchers conducted a selective review of the literature in order to identify effective consultation techniques (Edmunds et al., 2013a). They identified the following elements of consultation: 1) teaching/ instruction; 2) case review (either in the form of discussion or tape review); 3) self-evaluation; and 4) feedback.

In an exploratory study, researchers identified the content and distribution of consultation call topics provided to clinic supervisors in a large-scale dissemination of evidence-based treatments for children (Nadeem et al., 2013b). Results showed that the following topics were discussed on the calls: 1) case conceptualization and assessment (e.g., assessment tools, appropriateness of treatment protocols); 2) CBT model and techniques (general cognitive and/or behavioural principles, specific CBT techniques); 3) CBT supervision issues (e.g., agenda setting, clinician and supervisor comfort level with CBT); 4) fidelity and adaptation (e.g., discussion related to adherence to treatment manual and how to 
tailor treatment to meet client needs); 5) child and family engagement (e.g., discussion about children and parents' beliefs about treatment, how to engage families in treatment more effectively); 6) other clinically-oriented discussion (e.g., hospitalizations and suicide attempts); 7) programmatic or administrative issues (e.g., attendance, case completion criteria, data reporting); and 8) off task (e.g., weather, current events).

Using the same sample as the study described above (Nadeem et al., 2013b), a group of researchers examined the extent to which behavioural rehearsals, an indicator of active learning, facilitated clinician learning (Edmunds, 2013; Edmunds et al., 2013b). Results showed that significant time was spent in consultation discussing the core components of CBT for youth anxiety, with the majority of time spent discussing exposure tasks. Time was also spent discussing specific cases. Surprisingly, little time was devoted to behavioural rehearsals. Contrary to hypotheses, no significant relation was found between behavioural rehearsals during consultation sessions and therapist adherence, skill, self-efficacy, and satisfaction. However, clinician involvement moderated the relationship between behavioural rehearsals during consultation and skill acquisition, suggesting that actively involving clinicians in consultation may be an important part of the learning process. This study suggests that discussing central elements of a protocol and discussing cases may be among the primary elements of clinical consultation. Although it is likely to vary by context, the use of behavioural rehearsal may not be as common as expected. 
Another study explored therapist perspectives on effective consultation (Beidas et al., 2013). The researchers conducted semi-structured interviews with 50 therapists who had been trained two years prior in CBT. Therapists were asked the following questions: "What was most helpful about consultation? Would you have preferred a different type of consultation (e.g., more/less individual feedback, no recording, more/less role-playing)?" (p. 509). Three themes emerged from their interviews: 1) connectedness with other therapists and the consultant; 2) authentic interactions around actual cases; and 3) the responsiveness of the consultant to the needs of the individual therapists. The importance of connectedness, defined as "the experience of building supportive professional relationships" (p. 511) was identified as the overarching theme. Authenticity and responsiveness were conceptualized as sub-themes that facilitate connectedness. Therapists expressed that the group process, or the opportunities for interpersonal connectedness the group provided, was the most important component of clinical consultation. They maintained that connectedness reinforced their learning and clinical application of the material. Some therapists commented on the capacity of technology to influence their feelings of interpersonal connectedness - for some it enhanced connectedness, while for others it detracted. Additionally, therapists spoke about their preference for using actual case material, rather than role-plays or hypothetical cases, emphasizing the authenticity of this approach. In this context, role-plays were perceived as a threat to both connectedness and authenticity. They highlighted the helpfulness of discussing another therapist's case when it contained similar challenges to their own. Finally, therapists remarked that they appreciated the consultants' 
collaborative style and responsiveness to tailoring consultation to suit their needs and answer their questions. This tailoring of consultation helped ensure time was spent efficiently, and served to further reinforce feelings of connectedness among the therapists and the consultant. Therapists expressed that a limitation to the group format was that it did not provide adequate opportunity for individualized feedback, and that the desire for individualized feedback posed a threat to responsiveness. Together, these studies highlighted some of the consultation strategies and techniques that are likely to be present in the consultation calls of clinicians learning to deliver CPT.

\section{Group Delivery of Consultation}

In the current study, clinicians will receive consultation in a group format, which can be influenced by social facilitation, social comparison, and group dynamics. The delivery of consultation in a group format may therefore be an important contextual factor.

Groups are defined as two or more people interacting with one another (Shaw, 1981), and are characterized by the relationships among their members. The study of groups has long been of interest to psychologists (e.g., Johnson et al., 2006; Turner, 1987; Yalom, 1995). Understanding the functioning of groups requires studying how group members are related to one another and how they influence one another. A consistent focus of research has been to explain the reciprocal relationship between the individual and the group.

"Social facilitation" is a term that describes the phenomenon that the presence of others improves one's performance of a task (Triplett, 1898). However, 
under certain circumstances, the presence of others can hinder performance (Dashiell, 1930). Zajonc (1965) suggests that the presence of others increases arousal, which either enhances or impairs performance, depending on the task. The presence of arousal increases the likelihood of eliciting the individual's dominant response. For simple or well-rehearsed tasks, the presence of others enhances performance, whereas for complex tasks the presence of others can hinder performance.

One major contributor to this phenomenon is evaluation apprehension or concern for the perception of others (Cottrell, Wack, Sekerak, \& Rittle, 1968). Seta (1982) discovered that people perform best when they are working on a noncompetitive task with someone who is perceived to be slightly superior. The premise of social comparison theory (Festinger, 1954) is that people evaluate their own opinions and abilities against those of other group members who are perceived to be similar in ability. This comparative evaluation with similar others creates a tendency toward uniformity in groups. This is achieved by changing the standards of performance or by eliminating comparison with others by removing them from the group. In the present study, social comparison among clinicians may function to create standards for proficient delivery of CPT. Specifically, clinicians who are less proficient at the outset of consultation may become increasingly proficient as consultation progresses, in part because of the non-competitive nature of learning to deliver a therapy and from being in a group with more proficient clinicians. Kurt Lewin (1947) introduced the term "group dynamics" as part of his systematic study of how groups function. According to Lewin, groups have 
characteristics and properties that can be observed, measured, and predicted. Groups can be described in terms of the roles, norms, and cohesiveness of their members. A person's role refers to his or her set of expected behaviours. Norms refers to rules of conduct for group members. Group norms largely determine the flow of information within a group, which is determined by the group's communication network, an informal system that defines who can speak with whom. Difficulties in communication can reduce the effectiveness of groups. Cohesiveness is defined as "the resultant of all forces acting on the members to remain part of the group" (Festinger, Back, Schachter, Kelly, \& Thibaut, 1950). Cohesiveness is a group variable that is the product of many factors. When a group is highly cohesive its members respect each other and wish to continue associating as a group. Cohesiveness is based on perceived similarity among group members. Groups high in cohesiveness are highly satisfied by being together, whereas an external force must maintain groups low in cohesiveness. Accordingly, in the present study group cohesiveness may be an important contextual factor that influences learning during consultation.

A group of researchers developed a methodology to identify themes emerging in small group dynamics (Neuman, Assaf, \& Cohen, 2012). This methodological approach derives its data from transcriptions of group members' verbal utterances. Themes are then derived from the textual data. An important conceptual assumption is that the group as a whole is viewed as being qualitatively different than the sum of its parts. Foulkes (1964) called the web of communication and relationships among group members a "matrix" (p. 292). This approach posits 
that the group members and the group dynamics influence each other in a reciprocal manner.

\section{The Influence of Technology on Groups}

Meeting with the assistance of technology, rather than face-to-face, may influence the interpersonal processes of groups. Because team meetings using information and communication technologies (ICTs) are becoming increasingly common in many disciplines, the study of the impact of technology on group processes is a new area of research (e.g., Maruping \& Agarwal, 2004). Using ICTs as a means of communication between group members creates both opportunities and challenges. Previous research has shown that virtual teams that use computermediated communication are superior to teams that meet face-to-face in terms of brainstorming (Dennis \& Valacich, 1993) and decision-making (Sambamurthy, Poole, \& Kelly, 1993). At the same time, role ambiguity, low team commitment, and social loafing can be exacerbated in the virtual team context (O’Hara-Devereaux \& Johansen, 1994). Recent research on supervision (Brooks, Patterson, \& McKiernan, 2012) and consultation (Beidas et al., 2013) has highlighted some of the advantages and disadvantages of the technology on learning processes. Importantly, technology often facilitates group learning among clinicians who are geographically dispersed, contributing to connectedness. On the other hand, when technology is outdated or malfunctions it interferes with connectedness, and in turn with learning. In the current study, the use of technology is likely an important contextual factor. Specifically, given that the clinicians in this study are geographically dispersed, the use of technology facilitates their connection, which may facilitate their learning. 
Additionally, technological malfunctions during consultation may interfere with connectedness, in turn hindering learning.

\section{Present Study}

This study had the following primary objectives: (1a) to delineate the content and function of the primary elements of clinical consultation, including contextual factors and mechanisms; (1b) to present a theory of how consultation works; and, (1c) to identify context-mechanism-outcome configurations. This study also had the following secondary objectives: (2) to compare the proposed model of consultation to the dominant model of supervision (Milne et al., 2008); and, (3) to discuss whether Kolb's theory of experiential learning applies to consultation.

\section{Method}

\section{Participants}

This study involved the participation of clinicians and consultants.

Clinicians. The clinicians $(N=41$; see Table 1$)$ were mental healthcare providers over the age of 18 who attended one of five standardized, in-person CPT trainings. The inclusion criteria were: 1 ) being independently registered by a college to practice mental healthcare and having the provision of psychotherapy within one's scope of practice; 2) actively treating patients with PTSD; 3) consenting to be randomized to one of three post-workshop support modalities; 4) being willing to solicit participation of at least two patients diagnosed with PTSD for a full course (i.e., 12 sessions) of CPT; 5) consenting to have their CPT treatment sessions audio recorded; and, if applicable 6) consenting to have their consultation sessions audio recorded. No exclusion criteria were specified. 
Table 1. Clinician Demographics

\begin{tabular}{lrr}
\hline Characteristic & $n$ & $\%$ \\
\hline Sex & & \\
Male & 11 & 26.8 \\
Female & 30 & 73.2 \\
Age & & \\
30-39 & 9 & 22.0 \\
40-49 & 11 & 26.8 \\
50-59 & 15 & 36.6 \\
60-69 & 5 & 12.2 \\
70-79 & 1 & 2.4 \\
Ethnicity & & \\
White (Caucasian) & 23 & 95.8 \\
Mixed & 1 & 4.2 \\
Highest degree & & \\
BA, BS or equivalent & 4 & 9.8 \\
MA/MS & 8 & 19.5 \\
LCSW/MSW & 8 & 19.5 \\
PhD or PsyD & 15 & 36.6 \\
MD & 1 & 2.4 \\
Other & 5 & 12.2 \\
Profession & & \\
Nurse & 5 & 12.2 \\
Psychologist & 23 & 56.1 \\
Psychiatrist & 1 & 2.4 \\
Social Worker & 11 & 26.8 \\
Other & 1 & 2.4 \\
Years as a practicing mental health professional & & \\
1-5 years & 2 & 4.9 \\
6-10 years & 10 & 24.4 \\
11-20 years & 12 & 29.3 \\
More than 20 years & 17 & 41.5 \\
Current work affiliation & & \\
Operational Stress Injury Clinic (VAC) & 11 & 26.8 \\
Operational Trauma and Stress Support Clinic (CF) & 5 & 12.2 \\
Other Federal Health Service (DND, RCMP, Corrections, etc.) & 1 & 2.4 \\
Provincial Health Agency/Facility & 9 & 22.0 \\
Private Practice & 11 & 26.8 \\
Other & 4 & 9.8 \\
Current region of Canada & & \\
West (BC, AB, SK) & 21 & 51.2 \\
Central (MA, ON, QC) & 17 & 41.5 \\
East (NB, NS, PEI, NFLD) & 3 & 7.3 \\
Type of setting & & \\
\hline
\end{tabular}




\begin{tabular}{lrr}
\hline Urban & 34 & 82.9 \\
Suburban & 5 & 12.2 \\
Rural & 2 & 4.9 \\
\hline
\end{tabular}

Note. BA = Bachelor of Arts; $\mathrm{BS}=$ Bachelor of Science; MA = Master of Arts; MS = Master of Science; LCSW = Licensed Clinical Social Worker; MSW = Master of Social Work; PhD = Doctor of Philosophy; PsyD = Doctor of Psychology; MD = Doctor of Medicine; VAC = Veterans Affairs Canada; $\mathrm{CF}=$ Canadian Forces; $\mathrm{DND}=$ Department of National Defence; RCMP = Royal Canadian Mounted Police; $\mathrm{BC}=$ British Columbia, $\mathrm{AB}=$ Alberta, $\mathrm{SK}=$ Saskatchewan; $\mathrm{MA}=$ Manitoba, $\mathrm{ON}=$ Ontario, $\mathrm{QC}=$ Quebec; NB = New Brunswick; NS = Nova Scotia; PEI = Prince Edward Island; $\mathrm{NFLD}=$ Newfoundland ${ }^{\mathrm{a}} n=24$ 
With regard to the clinicians, $30(73.2 \%)$ were female. The mean age was 49.4 years $(S D=9.7$, range $=31$ to 74$)$. Fifteen clinicians did not report their ethnicity. Of the 24 that did, 23 (95.8\%) were Caucasian. With regard to the highest degree earned, the most common was a Doctor of Philosophy (PhD) or Doctor of Psychology (PsyD), which was reported by 15 (36.6\%) of the clinicians, followed by an Master of Arts (MA) or Master of Science (MS), which was reported by 8 (19.5\%) of the clinicians, and a Licensed Clinical Social Worker (LCSW) or Master of Social Work (MSW), which was reported by 8 (19.5\%) of the clinicians. The most common profession was psychologist, which was reported by 23 (56.1\%) of the clinicians, followed by social worker, which was reported by 11 (26.8\%) of the clinicians. With regard to the number of years as a practicing mental health professional, 17 (41.5\%) reported more than 20 years, 12 (29.3\%) reported between 11 and 20 years, 10 $(24.4 \%)$ reported 6 to 10 years, and $2(4.9 \%)$ reported 1 to 5 years. The two most common current work affiliations were Operational Stress Injury Clinic (Veterans Affairs Canada), and a provincial health agency or facility, which were each reported by $11(26.8 \%)$ of the clinicians. With regard to where the clinicians lived, the most common region of Canada was the western region, which was reported by 21 (51.2\%) of the clinicians, followed by the central region, which was reported by 17 (41.5\%) of the clinicians. The majority of the clinicians, 34 (82.9\%) reported practicing in an urban setting. Table 2 includes details about clinician psychotherapy and training experience. 
Table 2. Clinician Psychotherapy and Training Experience

\begin{tabular}{|c|c|c|}
\hline Response & $n$ & $\%$ \\
\hline \multicolumn{3}{|l|}{ Average hours per week providing psychotherapy to clients } \\
\hline $0-10$ & 5 & 12.2 \\
\hline $11-20$ & 17 & 41.5 \\
\hline $21-30$ & 16 & 39.0 \\
\hline $31+$ & 3 & 7.3 \\
\hline \multicolumn{3}{|l|}{ Approximate number of currently active psychotherapy cases } \\
\hline $0-10$ & 7 & 17.1 \\
\hline $11-20$ & 9 & 21.9 \\
\hline $21-30$ & 13 & 31.7 \\
\hline $31-40$ & 4 & 9.8 \\
\hline $41-50$ & 2 & 4.9 \\
\hline $51+$ & 6 & 14.6 \\
\hline \multicolumn{3}{|l|}{ Percentage of clients to which providing PTSD treatment } \\
\hline $0 \%$ & 2 & 4.9 \\
\hline $1-25 \%$ & 22 & 53.7 \\
\hline $26-50 \%$ & 6 & 14.6 \\
\hline $51-75 \%$ & 4 & 9.8 \\
\hline $76-100 \%$ & 7 & 17.1 \\
\hline \multicolumn{3}{|l|}{ Number of prior trainings in EBTs for anxiety disorders or PTSD } \\
\hline None & 7 & 17.1 \\
\hline 1 & 12 & 29.3 \\
\hline $2-3$ & 15 & 36.6 \\
\hline 4 or more & 7 & 17.1 \\
\hline \multicolumn{3}{|l|}{ Prior experience with CBT } \\
\hline None & 1 & 2.4 \\
\hline Some general knowledge, no courses or other training & 3 & 7.3 \\
\hline $\begin{array}{l}\text { One or two courses and/or trainings, no } \\
\text { supervision/consultation }\end{array}$ & 18 & 43.9 \\
\hline $\begin{array}{l}\text { supervision/consultation } \\
\text { Several courses and/or underwent supervised training or }\end{array}$ & 19 & 46.3 \\
\hline \multicolumn{3}{|l|}{ Types of training in psychotherapy currently being received ${ }^{a}$} \\
\hline Individual consultation & 6 & 15.0 \\
\hline Group consultation & 9 & 22.5 \\
\hline Peer supervision & 11 & 27.5 \\
\hline Self-directed (including online support) & 14 & 35.0 \\
\hline \multicolumn{3}{|l|}{$\begin{array}{l}\text { Previous experience with audio-/video-recorded feedback in } \\
\text { psychotherapy training }\end{array}$} \\
\hline Yes & 24 & 58.5 \\
\hline No & 17 & 41.5 \\
\hline
\end{tabular}


${ }^{\mathrm{a}} n=40$ 
Consultants. CPT consultants ( $N=6$; see Table 3 ) provided post-workshop consultation to the clinicians according to two different consultation models, which are described below. All CPT consultants were licensed mental healthcare clinicians who had extensive experience delivering CPT, and were designated as CPT consultants by the treatment authors based on consultation training and experience consulting/supervising in the model. The consultants were trained in a face-to-face consultant workshop provided by the treatment authors using the CPT consultants' manual. Consultants in the current study were expected to follow this manual for the duration of the study, and received one hour of telephone consultation per month with either Dr. Candice Monson or Dr. Shannon Wiltsey Stirman, two of the parent study's primary investigators. The telephone consultation was used to ensure the consultants were adhering to the consultation protocol, and to answer any questions that arose during consultation.

With regard to the consultants, 4 out of 6 completed the demographic survey. Among those who responded, 4 (100.0\%) were female. Three (75.0\%) were 40-49years-old, and 1 was 60-69-years-old. All 4 (100.0\%) of the consultants were Caucasian, and all $4(100.0 \%)$ reported being married. With regard to the highest degree earned, the most common was a PhD or PsyD, which was reported by 3 (75.0\%) of the consultants, followed by an LCSW or MSW, which was reported by 1 (25.0\%) of the consultants. Their professions included psychologist, which was reported by $3(75.0 \%)$ of the consultants, and social worker, which was reported by $1(25.0 \%)$ of the consultants. With regard to the number of years as a practicing mental health professional, $2(50.0 \%)$ reported 1 to 10 years, $1(25.0 \%)$ reported 
Table 3. Consultant Demographics $(n=4)$

\begin{tabular}{|c|c|c|}
\hline Characteristic & $n$ & $\%$ \\
\hline \multicolumn{3}{|l|}{ Sex } \\
\hline Female & 4 & 100.0 \\
\hline \multicolumn{3}{|l|}{ Age } \\
\hline $40-49$ & 3 & 75.0 \\
\hline $60-69$ & 1 & 25.0 \\
\hline \multicolumn{3}{|l|}{ Ethnicity } \\
\hline White (Caucasian) & 4 & 100.0 \\
\hline \multicolumn{3}{|l|}{ Current marital status } \\
\hline Married & 4 & 100.0 \\
\hline \multicolumn{3}{|l|}{ Highest degree } \\
\hline LCSW/MSW & 1 & 25.0 \\
\hline PhD or PsyD & 3 & 75.0 \\
\hline \multicolumn{3}{|l|}{ Profession } \\
\hline Psychologist & 3 & 75.0 \\
\hline Social Worker & 1 & 25.0 \\
\hline \multicolumn{3}{|c|}{ Years as a practicing mental health professional } \\
\hline $10-20$ years & 2 & 50.0 \\
\hline $21-30$ years & 1 & 25.0 \\
\hline More than 30 years & 1 & 25.0 \\
\hline \multicolumn{3}{|l|}{ Current work affiliation } \\
\hline VA Hospital or CBOC & 3 & 75.0 \\
\hline Other & 1 & 25.0 \\
\hline \multicolumn{3}{|l|}{ Years as a CPT consultant } \\
\hline $0-10$ & 3 & 75.0 \\
\hline $11-20$ & 0 & 0.0 \\
\hline $20+$ & 1 & 25.0 \\
\hline \multicolumn{3}{|c|}{ Training as a CPT workshop leader } \\
\hline Yes & 4 & 100.0 \\
\hline \multicolumn{3}{|c|}{ Training as a consultant or workshop leader in another EBT } \\
\hline Yes & 2 & 50.0 \\
\hline No & 2 & 50.0 \\
\hline \multirow{2}{*}{\multicolumn{3}{|c|}{$\begin{array}{l}\text { Prior supervised trainings in other EBTs for anxiety disorders or } \\
\text { PTSD }\end{array}$}} \\
\hline & & \\
\hline EMDR & 1 & 25.0 \\
\hline Prolonged Exposure & 4 & 100.0 \\
\hline Trauma-focused CBT & 1 & 25.0 \\
\hline CBT for PTSD & 1 & 25.0 \\
\hline \multicolumn{3}{|c|}{$\begin{array}{l}\text { Note. } \text { LCSW = Licensed Clinical Social Worker; MSW = Master of Social Work; PhD = } \\
\text { Doctor of Philosophy; PsyD = Doctor of Psychology; VA = Veterans Affairs; CBOC = } \\
\text { Community-Based Outpatient Clinic; CPT = Cognitive Processing Therapy; EBT = } \\
\text { evidence-based therapy; PTSD = posttraumatic stress disorder; EMDR = Eye } \\
\text { Movement Desensitization and Reprocessing }\end{array}$} \\
\hline
\end{tabular}


between 11 and 20 years, and $1(25.0 \%)$ reported more than 30 years. The most common current work affiliation was a Veterans Affairs Hospital or CommunityBased Outpatient Clinic, which was reported by 3 (75.0\%) of the consultants. With regard to the number of years as a CPT consultant, 3 (75.0\%) reported 0 to 10 years, and $1(25.0 \%)$ reported 20 or more years. All $4(100.0 \%)$ of the consultants reported they had training as a CPT workshop leader. Two (50.0\%) of the consultants reported that they had training as a consultant or workshop leader in another evidence-based therapy, and $2(50.0 \%)$ reported that they did not. With regard to prior supervised trainings in evidence-based therapies for other anxiety disorders or PTSD, the most common was Prolonged Exposure (Foa et al., 2007), which was reported by all 4 (100\%) of the consultants.

\section{Procedure}

Participant recruitment. Participant recruitment occurred in five rounds of solicitation. Each round occurred in conjunction with a CPT training workshop, all of which were led by Dr. Monson. The first round took place at Ryerson University in Toronto, Ontario, on March 21-22, 2012, and was open to clinicians practicing in the community. The second round took place at McMaster University in Hamilton, Ontario, on May 14-15, 2012, and was open to clinicians from St. Joseph's Healthcare. The third round took place in Halifax, Nova Scotia, on November 6-7, 2012. It was organized by the Operational Trauma and Stress Support Program of the Canadian Armed Forces Health Services, and was open to mental healthcare providers from the Canadian Forces. The fourth round took place in Edmonton, Alberta, on January 17-18, 2013. It was organized jointly by the Edmonton Operational Stress Injuries 
(OSI) clinic and several community clinics, and open to both clinicians who worked for the OSI clinic and clinicians who practiced in the community. The fifth round took place in Victoria, British Columbia, on February 6-7, 2013. It was organized by the Operational Trauma and Stress Support Program of the Canadian Armed Forces Health Services, and was open to mental healthcare providers from the Canadian Forces.

To recruit clinicians for the study prior to the scheduled workshop, the workshop organizers e-mailed workshop participants to solicit their participation in the study. Interested clinicians were asked to contact the study coordinator, who emailed them a link to the online consent and registration forms. Clinicians were given the option of faxing their completed forms, should they be unable or choose not to complete the registration form online.

Additionally, during the workshops, Dr. Monson gave a brief overview of the study, describing the consultation conditions, study requirements, and risks and benefits of participation. At the first workshop, the study coordinator and graduate students were available to answer questions about the study. During designated breaks, interested clinicians were invited to complete the informed consent process. At the other four workshops, where the study coordinator and graduate students were not present, clinicians were invited to contact the study coordinator. The study coordinator sent the consent form and baseline questionnaires to clinicians who were interested in enrolling.

Clinicians were responsible for recruiting patients from their workplaces. Clinicians used a script to solicit verbal consent from their patients. To protect 
patient privacy, no personally identifying patient data was collected. Clinicians signed a document confirming their receipt of verbal consent from all patients. Patients were told by the clinicians that they had the right to withdraw from the study at any time, and they could do so by informing their treating clinician that they no longer wished to participate. It was stressed that withdrawing from the study would not negatively affect their relationship with their therapist or any participating institutions.

Consultation. In the omnibus study, immediately following the CPT workshop, clinicians $(N=134)$ were randomized to one of three study conditions: fidelity monitoring only, standard consultation, and technology-enhanced consultation. After randomization, clinicians were assigned to a consultation group based on their availability. For a comparison of the three post-workshop conditions, see Appendix A. Only clinicians in the standard consultation condition were relevant to the current study.

All consultation sessions were conducted using GlobalMeet ${ }^{\circledR}$ (American Teleconferencing Services Ltd.), an international web conferencing service. Consultation calls were each 1-hour in duration and took place on a weekly basis for 26 weeks beginning 1 to 2 months after the workshops.

Clinicians randomized to the standard consultation were included in the current study. Consultation groups were comprised of one consultant and between 3 and $6(M=4)$ clinicians. In the standard condition, the primary purpose of the call was to discuss case material, as well as to address questions that arose in sessions and barriers to delivering CPT with fidelity. The content of the calls varied 
somewhat from week-to-week and was determined by events arising in clinicians' sessions. Consultants were encouraged to set an agenda at the outset of consultation, as well as to introduce the suggested format for presenting cases. Clinicians presented their cases verbally and received feedback from the consultant and other group members. Consultants were urged to invite group discussion and brainstorming, as well as to ensure clinicians had clear and specific feedback and a plan for the upcoming session. Clinicians also had access to handouts, manuals, and other support materials, including the CPT fidelity monitoring measure, a form developed by the CPT treatment developers to be used with their therapy. Roleplays and modeling were permitted but not required. Consultants were prohibited from discussing fidelity ratings or reviewing electronic worksheets that identified patients' stuck points. Additionally, the consultants encouraged the clinicians to participate actively by inviting them to ask questions, present cases, or contribute to another clinician's case conceptualization during each call.

\section{Materials}

All paper files (e.g., informed consent forms) were double-locked in Dr. Monson's research lab, at 105 Bond Street in Toronto, ON. All electronic data were stored on a password-secure server at Ryerson University. Clinicians used a digital audio recorder to record their CPT sessions.

\section{Realist Evaluation}

A research paradigm refers to the conceptual framework, or worldview, that guides the researcher's inquiry. Paradigms comprise ontology, epistemology, and methodology. Ontology refers to reality, epistemology refers to the relationship 
between the researcher and reality, and methodology refers to the techniques the researcher uses to apprehend that reality (Sobh \& Perry, 2006). In sum, a person's paradigm reflects his or her beliefs about knowledge creation and acquisition.

Realism is a model of scientific explanation that is distinct from the traditional poles of positivism and relativism (Pawson \& Tilley, 2008). According to the realist paradigm, there is an objective reality. Truth and knowledge exist independently of the human mind and can be accessed by employing the appropriate methodology and research tools (Flick, 2009).

In the positivist framework, the dominant model for scientific research, research findings are used to support or refute theories. In the realist framework, research findings are used to build theories (Sobh \& Perry, 2006). Social scientists often adopt the realist paradigm due to its usefulness in proposing and testing theories.

Pawson and Tilley (2008) explain that experimental research is largely concerned with discovering if a manipulable cause resulted in its intended effect, while realist evaluation is concerned with discovering how something works, or why a manipulable cause resulted in its intended effect. In other words, experimental research is primarily concerned with outcomes, while realist evaluation is primarily concerned with mechanisms and contextual factors. Therefore, conducting a realist evaluation involves identifying mechanisms (M) through which the intervention occurs, contextual factors (C) that influence the intervention's outcome, and describing how their combination leads to the intervention's desired outcomes (0). Fundamental to realist evaluation is the 
acknowledgement that the relationship between the mechanisms and outcome is contingent on the contextual factors. Pawson and Tilley (2008) recommend depicting this information as CMO configurations.

\section{Data Analysis}

Transcript Selection. Thirty audio recorded consultation calls were randomly selected and transcribed by either a professional transcriptionist or upper-level undergraduate student in psychology. To ensure maximum variation in sampling, 10 consultation calls from each of the three phases of consultation (beginning, middle, and end, divided evenly in thirds chronologically) were selected.

Coders. In addition to the author of this dissertation (ML), two graduate students in psychology with an interest in qualitative research coded the data (EV and SB). Both of the raters had previous experience conducting qualitative research. Specifically, EV volunteered as a research assistant on a study that employed qualitative analysis, and SB employed qualitative cod for her thesis. At the outset of the study, all of the coders read and discussed an article on the topic of how to conduct thematic analysis (Braun \& Clarke, 2006). One of the supervisory committee members of this dissertation (KM) provided feedback and advice while the data was being coded and interpreted. In this role, she provided support, encouraged reflection, and ensured that the approach to coding was methodologically rigorous and focused on answering the research questions. This committee member guided the coders through each phase of thematic analysis, coded a selection of the transcripts, and reviewed the coders' work. 
Reflexivity. Conducting qualitative research involves acknowledging that knowledge acquisition and construction are influenced by the researcher's approach to conducting the qualitative review. In other words, the researcher's ontology, epistemology, and methodology influence the process and results. The term "reflexivity" refers to the researcher's ongoing process of reflecting on his or her research (Alvesson \& Skoldburg, 2000). In the current project, the author of this dissertation (ML) engaged in methodological reflexivity (Raven, 2006) to ensure the analyses and interpretations were rigorous, and the conclusions drawn were consistent with the realist framework. This involved frequently revisiting the guidelines on how to conduct research from a realist perspective (Wong, Greenlagh, Westhorp, \& Pawson, 2014), and ensuring that the study's methodology was consistent with the realist philosophy of science. A defining feature of this methodological approach is its emphasis on understanding how contextual factors give rise to mechanisms that lead to the outcome of interest. In this tradition, mechanisms are regarded as tremendously important because they generate outcomes, and contexts can influence the processes through which an intervention produces an outcome. Accordingly, the data analysis focused on identifying the key contextual factors and mechanisms of consultation, as well as describing the relationship between them, and proposing how these mechanisms lead to proficient delivery of CPT. The model of consultation developed represents a theory to be tested empirically (Pawson \& Tilley, 1997).

Thematic Analysis. Thematic analysis, as described by Braun and Clarke (2006), is an appropriate analytic tool for research conducted from a realist 
perspective. Thematic analysis enables researchers to identify, analyze, and report patterns, or themes that are present within the data set. Defining a theme, Braun and Clarke (2006) write, "a theme captures something important about the data in relation to the research question, and represents some level of patterned response or meaning within the data set" (p. 82). As applied to the present study, the researcher will identify themes that reflect the primary elements of clinical consultation.

Braun and Clarke (2006) describe two ways of identifying themes within a data set: 1) inductive or 'bottom up,' and 2) deductive or 'top down'. The inductive approach requires the researcher to analyze the data without the use of a preexisting coding scheme, preconceptions about what he or she is likely to find, or a theoretical interest in a specific research question or topic. This approach to thematic analysis is highly data-driven. This differs from the deductive approach, which is guided by the researcher's theoretical perspective and interest in answering a specific research question. Compared to the inductive approach, the deductive approach is considerably more researcher-driven. The deductive approach is appropriate for projects such as this one, where the researcher is interested in a particular aspect of the data. In employing the deductive approach, the existing literature will be used to generate ideas for potential codes. Specifically, it is expected that the primary elements of supervision identified by Milne and colleagues (2008) will be present.

Braun and Clarke (2006) explain that themes can be identified at either a semantic or explicit level, or a latent or interpretive level. When thematic analysis is 
conducted at the semantic level, the researcher identifies themes by their surface or explicit meaning, and does not look for anything beyond what the data contain. This approach involves a progression from description to interpretation. The description requires the researcher to summarize and organize the data according to patterns in its semantic content. The interpretation requires the researcher to discuss the significance of those patterns, often in relation to the broader literature. In contrast, when thematic analysis is conducted at the latent level, the researcher moves beyond the semantic content of the data and explores the underlying assumptions, conceptualizations, and ideologies that are presumed to inform the semantic content of the data. For this project, a semantic approach to coding will be employed, such that the themes will be identified according to the explicit meaning of the data.

The researchers followed the six phases of data analysis according to thematic analysis as outlined by Braun and Clarke (2006). The analytic process is inherently recursive; therefore, movement back and forth between the phases is common.

Phase 1. In the first phase, the researchers familiarized themselves with the data. To do so, the writer of this dissertation randomly selected $20 \%$ of the transcripts and checked them back against the original audio recordings to identify errors in transcription. No major errors were identified (i.e. nothing that would change the meaning of a statement). Only minor errors were identified (i.e. typos). As the writer of this dissertation reviewed the calls, she noted initial ideas about patterns in the data. Additionally, the researchers read each of the transcripts with an eye toward identifying patterns in the data. After listening to the consultation 
calls and reviewing the transcripts, the researchers worked together to generate an initial list of ideas about patterns in the data.

Phase 2. In the second phase, the researchers coded the data and created the first draft of the codebook. The researchers began by coding six randomly selected transcripts, two from each phase of consultation. They independently reviewed the transcripts to identify codes, or patterned segments of text. After coding each transcript, everyone came together to discuss her findings. The team's findings were used to create the first draft of the codebook, a document that included definitions and examples of each of the codes. KM, the committee member with expertise in qualitative research, guided the coders through this process. The codebook was updated during every discussion, which occurred after each transcript was coded. The researchers focused on ensuring that codes were distinct from one another, and that they could be adequately operationalized and differentiated from one another.

Next, the researchers coded the entire data set. Candidate codes were added to and subtracted from the codebook throughout this process, and definitions were revised frequently to increase clarity and to help distinguish codes from one another. The act of coding consisted of highlighting segments of patterned text in electronic versions of the transcripts. To increase the clarity of the code, the text surrounding the code was preserved when the context was relevant. Keeping with Braun and Clarke's (2006) recommendations, the researchers coded for many potential themes, and extracts of data were coded inclusively. After the entire data set was initially coded, the researchers declared a complete draft codebook, which included a name, definition, inclusion/exclusion criteria, and examples of each code. 
Phase 3. In the third phase, the researchers searched for themes. To do so, they referred to the coded and collated data, and began to consider overarching themes into which the codes could be categorized. This phase focused on understanding how the coded data fits together, and whether it could be organized into themes. The researchers created a thematic map, a visual representation depicting the relationship between codes and themes, to sort the codes into themes. KM urged the researchers to carefully consider the research question at this stage.

Phase 4. In the fourth phase, the researchers reviewed the themes to assess the data's internal homogeneity (i.e., data categorized into the same code coheres together meaningfully) and external homogeneity (i.e., data categorized into different codes has clear and identifiable distinctions). They did so in two steps. The first step involved re-reading the collated data that comprise each theme and determining whether they formed a coherent pattern. The second step involved rereading the entire data set to confirm that the candidate themes accurately reflected the contents of the data set. This also involved finalizing the codebook by verifying the names, definitions, inclusion/ exclusion criteria, and examples. At this stage, the writer of this dissertation re-read the entire data set to identify data that had been missed in the previous round of coding, and to discard coded data that no longer fit with the updated coding scheme. Since the finalized codebook did not differ significantly from the rough codebook, the process of re-coding was one of finetuning. The other coders (EV and SB) verified their transcripts by reviewing the segments of text they had coded in the previous round of coding and re-coding them as needed according to the finalized codebook. 
Phase 5. In the fifth phase, the researchers defined and named the themes (see Results). The process of clearly defining and appropriately naming the themes involved several iterations. It consisted of a series of conversations between all of the coders (ML, EV, and SB) and the committee member (KM). The conversations focused on identifying the essence of each theme. Importantly, this phase also included discussions about what aspect of the data each theme captured, the boundaries of each theme, and how each theme functioned to answer the project's research questions.

Phase 6. In the sixth phase, the author of the dissertation (ML) conducted the final analysis of the data and reported the findings (see Results and Discussion). The final analysis included several conversations between the author (ML) and the committee member (KM) to ensure the analysis and reporting were consistent with the realist framework and met its requirements for reporting results (Greenlagh et al., 2014). It involved careful consideration of the function of each element of consultation, and how the elements of consultation function together. Vivid examples of each element of consultation were extracted from the transcripts and used to illustrate the findings. Several draft theories were created to explain how consultation works. After multiple iterations, a final theory of consultation was retained. Additionally, context-mechanism-outcome (CMO) configurations (Pawson, 2013; Pawson \& Tilley, 1997) were generated as causal explanations for how consultation works. The CMO configurations offer explanations that account for how the mechanisms lead to desired outcomes, and how the contextual factors influence outcomes. 


\section{Reliability}

Establishing inter-rater reliability is an integral component of conducting rigorous qualitative research (Morse, Barrett, Mayan, Olson, \& Spiers, 2002; Saladaña, 2009). Cohen's kappa coefficient is a statistic that measures inter-rater agreement for categorical items (Cohen, 1960) that is frequently used in qualitative research. Since Cohen's kappa coefficient takes into consideration the possibility that the inter-rater agreement occurred by chance, it is considered to be a more robust measure than percent agreement calculation (Gwet, 2014).

For this dissertation, inter-rater reliability was calculated at the transcript level, and was based on whether each of the coders identified each code described in the codebook as being present or absent in the identified transcript. Accordingly, the kappa coefficient reflects the proportion of agreement between coders according to the presence or absence of each code in each transcript averaged across all the transcripts. Kappa was found to be .80

\section{Results}

\section{Objective 1a}

The first aim of this dissertation was to identify and describe both the content and function of the primary elements of clinical consultation in a group of clinicians learning to deliver the CPT protocol. The contextual factors (see Appendix B) are presented followed by the primary elements of consultation (see Appendix C). 


\section{Contextual Factors}

The contextual factors refer to both social and physical characteristics of the environment in which the consultation calls occurred that likely influenced the process of consultation. The following contextual factors were identified: access to group support, clinician joins late, clinician does not have content to discuss, technological disruptions, and study participation. A rationale for their inclusion is included with each factor.

\section{General Contextual Factors}

Access to Group Support. Although the consultation process was designed to be a group experience, on several occasions clinicians were absent and only one clinician participated on the call. When this occurred, calls were typically much shorter in duration compared with when multiple group members were present. The consultant's statement, "It looks like you are the only one on the call" (Transcript 22, p. 1), exemplified the presence of this contextual factor. The following exchange also captured an instance whereby a clinician had access to individual support because the rest of the clinicians were absent:

Consultant: If I remember correctly [Clinician], I think that [Name X] and [Name Y] are not well. I think [Name Z] is not going to be with us going forward, and with this call two other people are out this week.

Clinician: Yeah, I think so.

Consultant: Okay.

Clinician: They were saying it was just going to be you and me. (Transcript 17, p. 1)

During calls such as this, the clinician who was present did not have the benefit of hearing about other clinicians' cases and offering his or her input. Hearing 
each other's challenges may have allowed each clinician to recognize and reveal his or her own challenges in delivering the intervention. This process may have been undermined when there was only one clinician on the call. Additionally, under these circumstances the clinician who was present did not have the opportunity to receive similar input from other clinicians. Since an individual consultation call may have provided fewer opportunities for reflection compared to a group consultation call, the lack of a group experience may have detracted from clinician learning. Furthermore, the absence of the other clinicians may have undermined feelings of connectedness between group members, which may have in turn detracted from the learning. On the other hand, consultation calls where only one clinician was present may have provided greater opportunity for that clinician to receive individualized attention or feedback, and to have his or her questions or concerns addressed without considering the needs of other clinicians. The receipt of individualized consultation input may have been advantageous for clinician learning.

Clinician joins late. During the calls, there were several instances of clinicians failing to be present at the beginning of the call, and then joining the call once the discussion was well underway. This contextual factor captured instances where a clinician was more than a few minutes late when he or she joined the call, or had missed a considerable amount of content when he or she joined. The consultant's statement, “Oh, it looks like we've got somebody. Hi, who joined?” (Transcript 4, p. 3) was an example of this contextual factor. The clinician's statement, made more than halfway into the call, "I just joined a few minutes ago. It's [Name X]" (Transcript 5, p. 11) was another example of this contextual factor. 
Additionally, the transcriptionist's note "New clinician joins" (Transcript 2, p. 10; Transcript 10, p. 9) was used to identify the presence of this contextual factor.

There were several ways in which a clinician joining the call late may have influenced the consultation process. First, the clinician who joined the call late did not have the benefit of hearing the material that was discussed previously on the consultation call. Clinicians who were late repeatedly, or who were so late that they missed a significant part of the discussion may have received a smaller dose of consultation compared with clinicians who were punctual. Since the success of consultation has been associated with dosing, clinicians who received less consultation may have benefitted less from consultation compared with clinicians who received a larger dose. Second, joining the call late temporarily disrupted the flow of the call and the conversation. This disruption may have caused participants to lose their train of thought, interfered with them developing and expressing fully their ideas, and may ultimately have detracted from the quality of the discussion at that time. Third, the disruption sometimes meant that the agenda had to be adjusted partway through the call to ensure the clinician who joined late was able to have his or her needs met by the call. This could have resulted in less time being spent on each agenda item than the consultant anticipated originally. In some cases, it may have left the consultant believing there was insufficient time to meet all the clinicians' needs. Fourth, it may have interfered with connectedness among the clinicians, and between the clinician who joined late and the consultant. The punctual participants may have felt annoyed by the interruption, and interpreted the clinician being late as a reflection that he or she did not value the group learning 
experience, which may have in turn detracted from the connectedness among that clinician and the rest of the group. Finally, if being late reflected a clinician's poor attitude toward consultation, the attitude may have functioned as a barrier to learning.

Clinician does not have content to discuss. Although it was more common toward the beginning of the consultation process than toward the middle or the end, there were examples from all of the phases of consultation where clinicians participated on the call, but did not have any questions to ask or case material to discuss. Commonly, this occurred either when a clinician had not yet begun working with a client, or when a clinician had not seen a client since the previous consultation call, and therefore did not have any new questions arise or case material to present. Other times, it occurred when the clinician reported that things were going smoothly with the client and that he or she did not have any questions. The clinician's statement, "I am in the process of picking someone up, but I haven't been able to see them yet. So, what I have got to contribute would be listening more than anything else at this point" (Transcript 12, p. 1), exemplified the presence of this contextual factor. The following quotation also demonstrated that the clinician did not have case material to discuss:

I'm somewhat like [Name X] here in that I have two patients in the clinical trial and I spoke about them in the last meeting, but I haven't met with them since that meeting. One of them is coming in here later today just like with [Name Y] and the other is coming in later this week. So I have nothing really new to update at this time. I think all of my questions were answered last week. (Transcript 18, p. 3)

Likely, clinicians who were not actively treating clients had less to contribute to other clinicians' cases than clinicians who were actively treating clients. Failing to 
bring his or her own cases may have limited the degree to which a clinician felt engaged in the process. It may also have impeded the development of a sense of connectedness among group members. Group members may have interpreted these clinicians' actions as an indication of not valuing the consultation process, or a lack of investment in learning. In the absence of feeling engaged and connected to other group members, a clinician who was not prepared may not have engaged in the reflection necessary for learning. Other clinicians may have distanced themselves from that clinician. Clinicians who failed repeatedly to bring material for discussion may have derived less benefit from the process of reflecting on other clinicians' challenges and offering suggestions compared with clinicians who brought material for discussion. The lack of reflection on their own cases may have made them less likely to reflect on, and offer suggestions regarding, other clinicians' cases. Ultimately, repeated failure to bring case material to discuss may have hindered both the clinician who failed to bring material and the group process overall.

Technological Disruptions. This contextual factor was considered to be present when technology malfunctioned during a call. Usually, technological disruptions had a minimally disruptive effect on the call, and a very small proportion of the total time on the call was spent talking about or resolving the technological issue. Some common examples of technological disruptions included difficulty logging onto GlobalMeet ${ }^{\circledR}$, difficulty hearing other participants on the call, intermittent reception, and difficulty with the "chat" function (i.e., the real-time transmission of text messages between participants). For example, the clinician's statement, "I was just having some technical difficulties at my end" (Transcript 5, p. 
11), was evidence of a technological disruption. The following interaction between a consultant and clinician also exemplified this contextual factor:

Consultant: Yeah - who is that that's on? Can you hear me?

Clinician X: It's [Name X]. Doesn't sound like she can hear you.

Consultant: Yeah, [Name Y] is that you?

Clinician Y: Yeah.

Consultant: Okay. Can you hear me okay?

Clinician Y: Yes.

Consultant: Okay, all right. It's - there's like a little bit of a delay or something, I guess. (Transcript $3, \mathrm{p} .2$ )

Minor technological disruptions such as these were common during the consultation calls. Occasionally, technology malfunctioned to the point that it interfered with the call, or a considerable amount of time was taken up discussing or trying to resolve the issues. Common examples included: repeated dropping of the call, the sound being so poor participants could not hear one another, or a significant portion of the audio recording being inaudible. For example, the transcriptionist's note after the consultant's statement in the following quotation was evidence of a major technological disruption: "That sounds fine. (Transcriptionist's note: Inaudible. Recording blip. Several clinicians advise that they are having difficulty hearing the consultant)" (Transcript 11, p. 3).

The following excerpt also reflected a major technological disruption: (Transcriptionist's note: Connection with consultant is lost at this point. Connection re-established at 17:13.)

Consultant: Hey, I do not know what happened, it just completely shut me off, I couldn't even get on the website it was bizarre - I lost you pretty early on 
into your discussion, so maybe if you don't mind backtracking (Transcript 29, p. 5).

Major technological disruptions may have undermined learning by interfering directly with the functioning of the consultation call. Technological disruptions may have interfered with the consultation process by contributing to participants' feelings of irritability and frustration, undermining participants' sense of cohesion, and occupying valuable time.

\section{Unique Contextual Factors}

Study participation. Discussion about the study was a reminder of the overarching context in which the consultation process occurred. When clinicians discussed the study, they often asked questions related to the study's policies (e.g., the number of patients they needed to enroll in the study; the consequences of failing to recruit the required number of cases; the consequences of failing to meet the requirements for fidelity to the protocol). They also often inquired about the study's logistics (e.g., how or when to submit relevant forms; how to upload audio recorded sessions), as well as the difference between the study conditions. The consultant's statement, "I am not sure what the decision has been for this study. So what I might do is e-mail [Name $\mathrm{X}$ ] because he is the one taking care of all the logistics and can probably let you know" (Transcript 3, p. 7), was evidence of this contextual factor.

The following statement made by a consultant also included discussion about the study:

That'll be interesting to see what happens with respect to the study because if he does go inpatient for a relatively brief period of time and gets stabilized, then I'm not sure exactly what happens in terms of what the study guidelines 
are in this specific study but that would be something we could definitely talk through with [Name X] or [Name Y]". (Transcript 8, p. 3)

The discussion about the study that occurred in the context of consultation reflected these clinicians' multiple roles - therapists, learners, and study participants. In addition to the demands that came with providing good clinical care and learning to deliver a new therapy, the clinicians had responsibilities as study participants. These responsibilities included completing forms, uploading sessions, and delivering the CPT protocol to the required two patients. It was difficult to know how the clinicians experienced their participation in the study. They may have experienced these additional responsibilities as stressful. Alternatively, study participation may have increased the sense of cohesion among clinicians who were in the same consultation groups, or who were at the same workplace and contributing to the creation of science.

On several occasions the consultants urged the clinicians to verify certain details about the study with the study coordinator. Doing so positioned someone other than the consultant as the expert, de-centralizing authority. Participating in consultation in the context of a study may differ significantly from practicing in a purely clinical context, where the consultant may be seen as more authoritative.

Finally, participants discussed the greater flexibility that might come from delivering CPT in a purely clinical context compared with some of the constraints that arose from learning the therapy in the context of a study (e.g., less pressure to see clients on a particular timeline; fewer forms to complete and upload). Although learning to deliver CPT in the context of a study may have increased the workload 
for clinicians, the increased accountability may have led to increased commitment and rigor, possibly contributing to greater fidelity to the protocol.

\section{Primary Elements of Consultation}

The results that follow describe what are thought to be the primary elements of clinical consultation. They are organized thematically according to their broader function in consultation. The results of objective $1 \mathrm{~b}$ describe how these elements are thought to function together.

\section{Organization}

The theme "Organization" is comprised of the elements "Attendance," "Agenda Setting," and "Structure. The theme encapsulates the administrative and coordinative tasks the consultants performed. Therefore, everything within this category was initiated or driven by the consultants. The organizational tasks provided an overall structure, kept the calls focused and efficient, and ensured the clinicians received the help they needed. Together, the organizational tasks functioned to create a framework that supported the learning. These tasks can be thought of as necessary but insufficient for learning to occur.

Attendance. "Attendance" refers to the process of taking a roll call to identify who was present on the call. It was considered to be present any time a statement was made identifying who was on the call, who would be joining the call late, or who was missing from the call. The following quotation from a consultant was an example of Attendance, "Good. All right, so we have [Name W], [Name X], and [Name Y]. And I'm looking back at my notes, I'm not recalling, is somebody out today? Is [Name Z] not coming today?" (Transcript 21, p. 1.) 
The following excerpt also exemplified Attendance:

Consultant: I do have all your names, so let me just check to see who is all on the call. Uh, I see on left hand side it looks like [Name V], are you here?

Clinician V: Yes I am, hello.

Consultant: And [Name W]?

Clinician W: Hi.

Consultant: Hi. And [Name X]?

Clinician X: Yes. Good morning.

Consultant: Okay, and that's all that's showing. I'm not seeing [Name Y] or [Name Z]. Are they on and I'm just not seeing them?

Clinician 3: No, I haven't heard them (Transcript 10, p. 1-2).

Taking attendance may have functioned to create a sense of order and accountability, facilitated cohesion among group members, and helped with setting an efficient agenda.

Agenda Setting. "Agenda Setting" refers to the consultants' efforts to organize how time on the call was spent. Typically, the consultants facilitated the process of setting the agenda near the beginning of the call, which created a structure for the remainder of the call. This process involved creating a list of items to be discussed based on the clinicians' needs and questions. The following quotation from a consultant exemplified Agenda Setting:

All right, so let's talk about making an agenda. [Name X] is looking at patients and so let's talk about if it's all right with you, talk about this person that [Name X] is going to see shortly to talk about her therapy and then let's talk about what about multiple traumas and what about they've been exposed to, what looks like very traumatizing events yet with capturing their attention they seem to want to talk about more organizational things which is not to say those are not very disturbing experiences too but...and maybe [Name Y's] not going to get on after all. (Transcript 9, p. 3) 
The following excerpt also exemplified Agenda Setting:

Consultant: Well, why don't we go ahead, and I can figure out from you both, how many sessions, if any, you were able to get under your belt this past week, and we can begin to start to set an agenda while we wait for those folks to join us. Let me just go down to my notes here. So [Name X], it looks like we left off that you were fairly early in the process?

Clinician X: Yes, I have had one more session since our last conference, so I've now had three sessions.

Consultant: So you're at session number three, [Name X]?

Clinician X: Yes.

Consultant: Just making a note of that. Okay, so we can chat about that session. And then I have a note that you had a woman that might be willing to start in April, the woman who served in Afghanistan, did that play out yet?

Clinician X: That's right. She was supposed to come in today, but had to cancel, so I might get started, possibly do the baseline session in one or two weeks.

Consultant: So we can talk about your session number three today. How about you, [Name Y]?

Clinician Y: I have managed to enroll one person, and I'm starting with him tomorrow morning.

Consultant: And then [Name Y], is that session tomorrow going to be the baseline session, or have you already completed that baseline?

Clinician Y: Done the baseline - it's going to be session number one.

Consultant: And [Name Z], how about you, how are things going for you?

Clinician Z: I'm up to, I just finished the sixth session with my one client, and I just started up another one here today, in terms of doing the baseline, just actually did that before our meeting here.

Consultant: So we'll get caught up on that. Well, I tell you what, in the interest of time then, why don't we back up and [Name X], do you want to chat about session number three, and tell us how it went, and if there's any questions about it? (Transcript 10, p. 2) 
Setting an agenda at the outset of the call may have ensured that all the clinicians had the opportunity to receive the help they needed. Setting the agenda may have served to clarify the clinicians' needs, which kept the discussion focused. Additionally, setting an agenda seems to have facilitated appropriate time management. Since the consultant was able to identify everything that needed to be addressed on the call at the outset, she could prioritize urgent or time-sensitive issues, and segue to the next agenda item when she was sure that an agenda item had been adequately addressed.

Structure. "Structure" refers to instances whereby the consultant led or directed the flow of the call. Typically, this involved segueing from one person or topic to another, and ensuring every clinician had the opportunity to present his or her case and have his or her questions answered. Although this occurred infrequently, there were times when the consultant had to ensure one clinician did not monopolize the call. In the first few consultation sessions, setting the structure involved giving instructions to clinicians about how to present their cases and what information to include in the case presentations. Over time, the clinicians became socialized to this structure and presented their cases in this format without prompting. In the following quotation, which was extracted from a call that occurred near the beginning of the consultation process, the consultant created structure by outlining the format she wanted the clinicians to use when presenting cases:

What I'd like to spend the call on, which normally is what we'll do on every call, is I'll ask you to give me a brief overview of your caseload. The first time that you introduce a client, if you'll tell me their age and gender and what their index trauma is that you're working on in CPT, that is how I will keep notes on that person. So I don't ever get their name. I just do it by age and gender. That typically is okay. Every now and then I'll have somebody with 
two 49-year-old females and then we're just hoping that the index trauma isn't the same, and if they are, then we'll find some other identifier. And then, on a weekly basis, when you check in at the beginning of the calls, I'll ask you to give me an update on the clients that you're seeing, including what their session number is. I usually also ask for the PCL score, just because it gives me an idea of what their symptoms are looking like. And then I'll ask you for whatever questions you have for the call today. And then once I do check in with everybody, I'll go back and start to respond to those questions one by one, after check in. That allows me to budget my time a little bit better. So please any questions that you do have, feel free to put them out there when you're checking in because sometimes I won't leave time at the end of the call for just anything else. Sometimes we'll have time for that, but other times not. (Transcript 7, p. 3-4)

The consultant's statement, "I can address that a little bit as well. Thank you for that. And that brings us to [Name X]," (Transcript 1, p. 4) is an example of how the consultant maintained structure by assuring one clinician her question would be addressed, and then segueing to the next clinician.

Consultants' efforts to set or maintain structure functioned to keep the process of consultation efficient and effective. It ensured that all of the items the clinicians put on the agenda got addressed, and permitted time for the consultants to add topics they considered to be important. Sometimes consultants made notes about things clinicians needed help, and then returned to those topics toward the end of the call. Doing so permitted consultants to prioritize discussion of the most urgent or important issues. Additionally, when consultants segued from one topic to the next, it prevented the belaboring of certain topics. All of the consultants ensured time on the calls was not wasted or spent discussing things that were off topic or irrelevant. Occasionally, time was spent making pleasantries, discussing the weather, or dates when participants would be absent. This typically occurred either at the beginning or the end of a call, and did not take up considerable time. 
In sum, setting or maintaining structure was considered to position the consultant as the authority, ensured all the agenda items were addressed, and contributed to the effectiveness and efficiency of the consultation calls. The calls tended to have a sense of focus and rigor to them, and this was achieved largely as a result of the consultants' setting or maintaining call structure.

\section{Knowledge Consolidation}

Knowledge Consolidation was found to be an overarching function of consultation. This central theme is comprised of the elements "Asking a Factual Question" and "Information-Based Teaching". Asking a Factual Question captures questions posed by clinicians, and Information-Based Teaching typically captures the consultants' responses. Together, these elements of consultation seemed to capture the process through which clinicians refined and solidified their understanding of CPT. These elements of consultation likely functioned to increase clinicians' declarative knowledge of CPT. Although many elements of consultation likely contributed to knowledge consolidation, the elements of consultation categorized in this way contributed most significantly to this process.

Asking a Factual Question. This element refers to instances of clinicians asking about factual information related to CPT. It captures clinicians' seeking specific information in either a direct or indirect manner. The questions posed pertained to didactic content delivered in the workshop or available in the CPT manual. Clinicians inquired about the following topics: how to identify the index event when clients have multiple traumas; which events qualify as traumatic; what to assess before beginning treatment; the content of various sessions and what to 
focus on in various sessions; whether the addition of certain techniques is considered a deviation from the protocol; whether clients with substance use issues are suitable for CPT; the theory underlying CPT; the terminology of CPT; and, how to deliver various components of CPT. Typically, the responses to these questions were factual. The clinicians' questions, "I'm just wondering if you can remind me what a stuck point would sound like if it was an assimilated one versus an accommodated one," (Transcript 1, p. 12) and "If somebody is progressing really well, do you do all twelve sessions?" (Transcript 14, p. 10) both exemplified Asking a Factual Question.

Asking a factual question is thought to be the clinician-driven component of knowledge consolidation. Achieving fidelity to the protocol required gaining a thorough understanding of the protocol's content and the way in which the treatment developers intended for it to be delivered. Asking a factual question seemed to suggest a clinician may be engaging with the learning process by reflecting on his or her delivery of the protocol.

Information-Based Teaching. "Information-Based Teaching" refers to instances whereby consultants impart information related to PTSD (e.g., symptoms, PCL scores) or CPT (e.g., elements of the protocol, information about the protocol, or the theory underlying CPT). Frequently, it includes offering an explanation, rationale, or the reasoning for something. Information-Based Teaching is often a consultant's response to a clinician's factual question. A defining feature of this element of consultation is that the content the consultant communicates is available elsewhere (e.g., the CPT manual, published manuscripts, training materials). 
Many topics were covered as part of Information-Based Teaching. The following were some of the most common: the meaning of various terms (e.g., impact statement, trauma account, stuck point); the content and focus of different sessions; the typical course of PTSD symptoms; how to read and interpret PCL scores; the difference between CPT and CPT-C; how CPT is similar to and different from other PTSD treatments; the difference between assimilated and overaccommodated stuck points; the role of natural emotion in relation to the model of CPT; and, how and when to use various worksheets (e.g., ABC sheets). The following quotations exemplified consultants engaged in Information-Based Teaching:

Just to kind of review session one, the clients aren't really getting to say much of anything. If you're getting any kind of stuck points in session one, that's great; you are already ahead of the game. Really there's not a whole lot of information you're getting besides just beginning to build rapport, helping them see hope, you know, instilling some maybe understanding of the rationale so you can get some motivation. In session two, it really starts to get more information from the client but not in terms of challenging. At this point in session two all we're really doing is trying to understand the stuck points. (Transcript 1, p. 7)

Then at session three you'll assign the first written trauma narrative, so that's when they actually write about their story, exactly as you were saying, and you want that to be the story of the index trauma because that's their opportunity to feel those natural emotions. (Transcript 2, p. 7)

The function of information-based teaching seemed to reinforce what was learned in the workshop in order to help clinicians learn the therapy. As the clinicians delivered the therapy, they inquired about factual matters related to CPT. Through the process of reflecting, inquiring, and learning more about CPT, the clinicians seemed to deepen their understanding of the conceptual and technical aspects of the treatment. Given the importance of understanding a treatment in order to deliver it proficiently, increasing clinicians' knowledge of the therapy 
beyond what was learned in the workshop may have been an important component of clinical consultation.

\section{Case Conceptualization}

Along with Knowledge Consolidation, Case Conceptualization was found to be an overarching function of consultation. It refers to instances of discussing case material, and includes presenting background information, conceptualizing client problems, and determining how to intervene. This element of consultation also includes descriptions of what occurred during therapy sessions, and can refer to something said by either a clinician or a consultant, or a conversation between multiple group members.

When presenting cases, clinicians typically relayed the following information to the group: client demographics; relevant background information; index trauma; PCL scores; and session number. Many topics were covered as part of Case Conceptualization. The following topics were discussed frequently: how to conceptualize PTSD and its comorbidities (e.g., depression, substance abuse, BPD); key cognitions and behaviours that are maintaining the client's PTSD (e.g., the function of behaviours such as dissociation and substance abuse); the role of avoidance in maintaining clients' PTSD; clients' individualized stuck points; and, what central elements of the protocol (e.g., trauma account, $\mathrm{ABC}$ sheets) revealed about clients. Case Conceptualization often involved discussing how various elements of the case were connected, or identifying which of the clients' key cognitions, behaviours, or environmental factors ought to be the focus of the intervention. 
Additionally, this element of consultation included instances of clinicians

describing their reactions to their clients, and discussing how to understand and make use of those reactions in session. How the clinician handled challenges that occurred in session was also discussed. The quotation below is an example of a clinician offering his own Case Conceptualization:

My other guy, we've taken our first out of three sessions to step out of the protocol. He's a little bit more challenging because a lot of his stuck points are around childhood abuse so family of origin, mom was really invalidating, dad was physically abusive and he had an interaction with his father where at age 18 his father kicked him out of the house and just physically pushed him out of the house and he never went back. So, he went back to help his dad do something this last week and his 80-year-old father punched him and then tried to get him out of the house and it replayed some of the stuck points. So, we talked about how he coped with that moment, and what was interesting was, even though we stepped out of the protocol he was talking about the skills that he'd learned I think while on session eight with him and what he said was, "You know I kept saying to myself I don't need to be afraid of him, he can't hurt me anymore. I can tell him to not treat me this way and I have the right to do that." So he said, "My internal dialogue was different. Like I was challenging those old stuck points that we've been working on" and what he realized from the conversation with his father and how abusive his father was, even though I think his father is in his late 70s or 80s, is that these are the things that cause him to have difficulties at work because his stuck points are he keeps expecting anybody in a position of authority to treat him this way. So, even though we stepped out of the protocol, we were actually using all of the stuff in the protocol and he said, "That's the first time I ever talked back to my dad. It's the first time that I sort of said I will not let you bully me or treat me this way. I am worth it and what you say about me doesn't define me." Part of what he over did was, he realized that he was hoping that his dad's reaction would be different. He put that sort of external logistic control where, if my dad backs down, if my dad says, "You're right I abused you," that the world would be okay and his dad just kicked him out again and said, "You're dead to me." So we're dealing with some of the grief issues and I need to go back in the next session and talk about how when we do something for a result of change in the other person, and use that as the determinant of whether or not we were successful, there's a stuck point there that we need to work on. (Transcript 16, p. 3)

The following excerpt exemplified a collaborative Case Conceptualization

between a consultant and clinician: 
Consultant: Okay, and so tell us how did, we'll start with your 38-year-old, how did that turn out in the end? Do you remember what her final PCL was?

(Transcriptionist's note: Other clinician joins meeting.)

Clinician: Her final PCL was basically 54.

Consultant: What did it start out as?

Clinician: Ah man, we started out, it was high, we started out it was 66, and basically it stayed high right till that seventh session where we kind of discussed it, and for eight, nine, 10,11,12, it basically floated anywhere between about 49 and 54, 55 .

Consultant: So, tell us a little bit about how you wrapped up with her.

Clinician: Oh, well you know, it was really interesting, it was a bit of a surprise because basically for that session, 10 and 11 , she was really, well, you know, to the point where...Well, I think I need some more sessions - do you think perhaps we could go over some other ones again, and really just different things coming in and out, and just throwing some things out there. But we really held to the fact that we're going to do the 12 , and we got some follow up things happening, and I have to say that her final impact statement was, it surprised me.

Consultant: Surprised you how?

Clinician: It was quite empowering for her, compared to the first one, and you know we really had a good discussion around the differences in that impact statement. (Transcript 29, p. 1)

Case conceptualization is thought to function as an active learning strategy

that may facilitate the process of translating the declarative knowledge that clinicians begin to acquire during the workshop into procedural knowledge that they begin to develop by delivering the therapy. This is thought to occur in several ways. First, the standardized format for presenting cases seemed to orient clinicians to the clinical details that are of greatest importance. For example, this format taught clinicians to administer routinely, and interpret correctly, the PCL, consider carefully various forms of avoidance, and identify stuck points. Second, discussing 
cases may have helped clinicians connect theory with practice by solidifying the content that was presented during the workshops. In this way, case conceptualization likely contributed to knowledge consolidation. Third, during this process clinicians seemed to reflect on what occurred in sessions, which may have also assisted with knowledge consolidation. For example, discussing clients' stuck points with the group likely helped clinicians understand what a stuck point is. When a clinician heard a potential stuck point in session and then brought it to the group to discuss, the process of reflection and discussion likely helped clarify both the concept of a stuck point and how that particular stuck point was maintaining a client's PTSD. In a similar way, this process may have helped clinicians deepen their understanding of PTSD, the theory underlying CPT and how it gets applied to clinical cases, and how to make use of the worksheets and assignments that are specific to the therapy (e.g., trauma account, $\mathrm{ABC}$ sheets). Fourth, discussing case material seemed to provide an opportunity for the clinician to explore various hypotheses about what was going on with the client and receive input from the consultant and other participants.

\section{Asking for Help}

The standalone element "Asking for Help" refers to instances whereby clinicians asked consultants about suggestions for how to approach a situation or deal with a clinical issue. In some instances the request for help was direct, while in other instances it was implied. This element differs from "Asking a Factual Question," which refers to instances of clinicians asking about factual information delivered in the workshop or available in the CPT manual. In contrast, Asking for Help refers to 
clinicians asking about what to do in a particular situation. Typically, clinicians asked for help conceptualizing cases, adapting the therapy to meet their clients' needs, or determining what course of action to take with a client. Typical examples of Asking for Help included the following: how to adapt the therapy to address a particular client concern, while remaining adherent to the model; how to help patients who were emotionally dysregulated engage with the cognitive work; how to prevent themselves from adopting the client's maladaptive belief system in order to challenge it; what to do when PCL scores were not decreasing; and what to do when the clinician did not correctly assign the trauma narrative. Sometimes, clinicians asked for feedback on whether the consultant would have handled a challenging situation in a similar manner. The clinician's question, “...Do I get him to do that as his homework again, or would I get him to do that right in session?" (Transcript 4, p.16) exemplified Asking for Help. In the following example, the clinician asked for help with Socratic questioning to prevent herself from sharing the client's stuck points:

What I'm finding though, is that I guess I need some assistance or suggestions on Socratic questioning, because internally I find myself getting sucked in and sharing his over-accommodations. He is so articulate in what he is writing down that I'm kind of sharing the over-accommodations with him. So, I'm struggling to get in this Socratic questioning, because when I read what he has down, some of the time I'm going, internally, yeah, I guess I would think the same thing. (Transcript 10, p. 3)

Asking for Help seemed to reflect clinicians' engagement in the learning process. The clinicians' articulation of what they needed help with suggested they were reflecting on what was going well in session and what was not. It also seemed 
to reflect that the clinicians trusted the consultant; their willingness to reach out to the consultant suggested a sense of connectedness between them.

Finally, the act of asking for help from the consultants placed the clinicians in the position of actively guiding their learning process. Through directing their learning, the clinicians may have come to feel more engaged in the learning process. The consultants' willingness to address the clinicians' needs seemed to reflect a sense of collegiality. Although the consultants were positioned as the experts and the clinicians as the learners, in this instance the clinician may have been seen as an expert in his or her own case and in identifying his or her needs, and the consultant may have been invited to contribute her expertise as needed. This seemed to foster a collaborative learning environment.

\section{Directive Instruction}

The theme "Directive Instruction" is comprised of the elements "Giving Advice" and "Modeling". This theme encapsulates the ways in which the consultants instructed the clinicians to proceed. Directive Instruction was action-oriented, and included explicit steps for the clinicians to take with their clients. Therefore, everything within this category was initiated or driven by the consultants.

Giving Advice. The element "Giving Advice" refers to instances of the consultant giving instructions, making recommendations, or offering guidance or suggestions to a clinician for how to proceed. This element differs from "Information-Based Teaching," which refers to instances whereby consultants impart information related to PTSD or CPT that is available elsewhere (e.g., CPT manual, workshop materials). In contrast, Giving Advice refers to consultants 
making recommendations about what a clinician should do in a particular situation.

The consultant's advice was typically given in a directive manner, and included concrete steps for the clinician to take. Two types of advice giving were conceptualized as comprising this element of consultation: 1) specific advice on the case being discussed, and 2) general advice about how to handle particular types of clients, situations, or issues. Consultants frequently gave advice on the following topics: when to accept a client's perception and when to challenge a client's stuck points; how to teach clients to complete their worksheets accurately; how to ensure clients completed the PCL in relation to their index trauma; how and why to prioritize targeting clients' assimilated stuck points; how to use various worksheets during the termination phase of therapy; what to put on the stuck point log; how to identify the index trauma; what to target first in therapy; what to do if a client puts an emotion on their stuck point log; how to address emotional dysregulation in session in a manner that is consistent with the model; and how to provide support during the reading of a trauma account. The following statements made by consultants exemplified Giving Advice:

One of the things that might help everyone with that - instead of feeling like you're doing, "Okay, it's session one and I have to do this, this and this". As clinicians, think about why you're doing it. What is the purpose of the assignment? What is the purpose of the thing that you are doing? (Transcript 6, p. 19)

I would really use this worksheet and this opportunity as a place to help him articulate that. Because he's already identified the emotion, and then as soon as he says it, go ahead and add it to his stuck point log. (Transcript 10, p. 8)

Giving advice seemed to function to provide clinicians with concrete steps to take when delivering CPT. When clinicians were unsure how to proceed, steps were 
laid out by the consultants. Giving advice is postulated to reinforce the clinicians' perceptions of the consultant as the expert. Their confidence in the consultant may have alleviated the pressure they felt to make clinical decisions on issues they did not yet feel ready to address independently. Therefore, giving advice may obviate this aspect of clinical care and function as support to the clinicians.

It is thought that consultants gave advice regarding issues they considered of significant importance. Doing so may have signaled the importance of the issue to the clinician, and demonstrated how to address it and similar issues in the future. Giving advice may have helped the clinician understand how to deliver the protocol. Providing concrete steps for the clinician to follow may have increased the likelihood that the clinician would deliver the treatment in a manner that is consistent with the model.

Modeling. The element "Modeling" refers to instances of consultants offering clinicians suggestions for what to say. For example, consultants modeled the use of precise language around sensitive topics, and how to talk to clients about their remaining stuck points during the last session of therapy. The following statements from consultants exemplified the use of Modeling:

What I might say is something like, "Okay so today we're going to review what you did, and I want to take a look at your ABC sheet. I'll look at your $\mathrm{ABC}$ sheet, but I also need to make sure we spend some time, probably the last 10 or 15 minutes talking about your next assignments." (Transcript 1, p. 9)

On that day with the knife or whatever event and say, "Let's specify even more, within this whole marriage, let's talk about that big day, the one that whatever happened, how do you think that happened that day?" (Transcript 17, p. 10-11) 
Modeling is thought to help clinicians to deliver components of the intervention with fidelity. Hearing the consultant model the conversation may have removed the clinicians' hesitation or doubt about how to handle the situation, and given them the confidence to respond as the consultant did. Hearing the desired behaviour modeled may have increased the likelihood the clinicians would engage in that behaviour in subsequent sessions. Modeling may have enhanced both declarative and procedural knowledge, and seems to have functioned as a bridge between the two. Due to its clarity and specificity, modeling may have been a particularly effective consultation strategy. It may have taught the clinicians how to address complex issues in a nuanced way by showing them exactly how to navigate particular challenges. Knowing how an expert would have handled a situation may have increased clinicians' confidence, and the demonstration of a strategy gave them a plan for how to address the issue clinically.

\section{Non-Directive Instruction}

The theme "Non-Directive Instruction" is comprised of the elements "Sharing a Personal Experience" and "Socratic Questioning". This theme encapsulates the ways in which the consultants encouraged the clinicians to reflect in order to determine how to proceed. Everything within this category was initiated or driven by the consultants.

Sharing a Personal Experience. This element refers to instances of consultants describing subjective or personal knowledge gained in the role of clinician, expert, or trainer. When the consultant shared a personal experience, she usually described ideas, insights, or suggestions not found in the CPT manual. 
Typically, these included the consultant discussing her own cases, personal examples, stories, anecdotes, or offering an explanation of her usual practice. These experiences had the feeling of being "tricks of the trade". Consultants often shared their experiences with regard to the following: how to generate stuck points with clients who have a hard time generating them on their own, and how to be collaborative when working with a client to generate stuck points; how to work effectively with people who are abusing substances, and how to conceptualize accurately their substance use; the benefits of working within a structured protocol; the extent to which patients have to grasp a concept before moving onto the next one; how to adapt the protocol for clients with particular needs (e.g., low literacy levels); less common ways in which avoidance can manifest (e.g., excessive sex, exercise, work); and, how to work effectively with difficult patients. The following examples shared by consultants illustrated Sharing a Personal Experience:

We've even had, I had a gentleman recently - his trauma was childhood and he used his experience to speak to gangs and try to reduce gang-related violence through the context of his job, and so he had actually spoken about his trauma in significant detail. So, that absolutely can happen. It was interesting when we went through the therapy - he was emotional when he got to disclosing details of his trauma, and I had anticipated that he wouldn't be, just because he does it so often quite publicly, and what I found was there was a bit of a different between kind of a public version and the way we specifically asked him to do it in CPT. So, I don't know whether that will play out with your client in terms of moving through that avoidance and so forth. They may just have asked her to tell them about it but not necessarily get into the details, or maybe that really get into the meaningfulness of the trauma, which I think catches a lot of that natural emotion. (Transcript 17, p. 2)

Yeah, I mean um, I'm working with a guy right now where we've been talking about power and control for the last two or three sessions, and we have a couple more sessions to go, and we'll still be talking about power and control. I mean he had power and control issues before the trauma - he's got power and control issues now. And, um I think power and control is a really 
interesting thing that people with anxiety disorders, especially if they were pre-existing, I think pre-existing he had you know, kind of some GAD and there's just a lot of wanting to control his environment - very "type a". And so I don't expect that CPT is going to cure that - I think that that's going to be, he's a little bit hardwired that way and it's a long time of learning, and so to me that's just something you develop, that ability to kind of keep that need for control in check and like really reminding yourself that that's impossible to have a hundred percent control over things...For my guy, the power and control was there before the trauma ever laid on top of it. But when he finally you know, was really faced with the fact that okay I do not have control, um, it was just overwhelming to him. But now we're having to deal with the fact that he wants to have power and control and always has, um so it's, over everything so it's very, um, it's become kind of bigger than PTSD. (Transcript 23, p. 13)

Consultants' sharing personal experiences may function to illustrate how to apply various elements of the CPT protocol (e.g., identifying and challenging assimilated stuck points). Much of the nuance of delivering a therapy cannot be communicated through information-based teaching, and sharing personal experiences seemed to elucidate how to deliver CPT. For example, consultants often told clinicians how they handled certain logistical issues (e.g., how they structured sessions; how and when they added stuck points to the stuck point log). By sharing their experiences with the clinicians, the consultants seemed to make the therapy more accessible, modeling how to apply the theory in a thoughtful way. Through sharing anecdotes about their clinical work and their own learning process, the clinicians may have come to view the consultants as more authentic individuals and credible clinicians. They seemed to become more relatable to the clinicians, which in turn increased their sense of connectedness.

Consultants' sharing personal experiences is thought to play an integral role in clinicians' acquisition of procedural knowledge. As consultants described how they approached various challenges, this modeled to clinicians how to approach 
similar challenges. For example, when consultants shared their personal experiences in the context of case conceptualization, consultants often drew parallels between their own clients and the client being presented. This helped deepen clinicians' understanding of different symptom presentations, various ways that avoidance manifests, and right-size expectations for symptom change throughout the course of therapy. Sharing a personal experience seemed to complement information-based teaching by illustrating how to apply the theory and protocol across a variety of situations, and solidified the consultant in the expert role.

Socratic Questioning. This element refers to instances in which the consultant encourages the clinician to reflect on a central concept or idea, application of the protocol, or the particularities of how to execute a component of the protocol. The following topics were explored through the use of Socratic Questioning: the importance of imposing structure on the sessions; the need to deliver CPT as prescribed in the manual; and, the role of the structured protocol in helping clients overcome avoidance. Socratic Questioning was also used to elicit clinicians' thoughts on their cases. For example, consultants probed clinicians to consider the following: how to get clients to articulate the downsides of avoidance; what stuck points are likely to be contained in a client's trauma account; and, how to understand various changes in the client's presentation. Finally, Socratic Questioning was used to query about clinicians' decision-making process. For example, due to her concerns that her client had not grasped central concepts, a clinician was considering going back to the beginning of the protocol and re-doing 
the first few sessions. Socratic Questioning was used to explore the clinician's thinking around this issue, and to help her arrive at a decision about how to proceed. The consultant's statement, "How would you say that to a client? I mean in this situation, how you would guide her through that?" (Transcript 8, p. 7) exemplified the use of Socratic Questioning.

Additionally, the following example illustrated the use of Socratic Questioning:

What are some of your thoughts about conceptualizing those problems within this model? Where might be some of the places you go and thinking about this case, sorting out these problems, how it fits, and how CPT could approach it? (Transcript 9, p. 5)

Socratic questioning may have functioned to enhance reflection, which is thought to facilitate knowledge consolidation and case conceptualization. The consultants appeared to use Socratic questioning when there was a need for the clinician to make an important decision. In some instances, the consultant used Socratic questioning to function as a correction - to help the clinician realize what he or she had done incorrectly and to articulate what he or she ought to have done instead. Through careful use of Socratic questioning, consultants led clinicians through a process of their own guided discovery, which is a mainstay way that clinicians are instructed to engage with clients. Encouraging clinicians to reflect may have contributed to deeper processing compared to elements of consultation where consultants imparted information (e.g., Information-Based Teaching) or instructed clinicians how to proceed (e.g., Giving Advice).

\section{Provision of Feedback}


The theme "Provision of Feedback" is comprised of the elements "Correction" and "Praise". This theme encapsulates the elements where the consultants communicate to the clinicians what they are doing correctly and incorrectly.

Correction. "Correction" refers to an instance whereby the consultant highlights something the clinician did that was incorrect, or contrary to the CPT protocol. Typically, this included pointing out an error in the clinician's understanding, conceptualization, or delivery of a particular element of the protocol. The following excerpts illustrated this element of consultation:

Consultant: Yeah, and did you give your client the actual practice assignment, the actual handout practice assignment?

Clinician: As a piece of paper?

Consultant: Yeah did you-

Clinician: I didn't. No, I didn't see that.

Consultant: Okay. For each, do you have the patient materials available to you?

Clinician: Yeah, I do.

Consultant: It should be, each session should have an actual written practice assignment to that. (Transcript 8, p. 14)

Clinician: [Name X], I'm only seeing my clients every second week or so.

Consultant: Ooh, that's not good. That encourages a lot of avoidance. We actually don't have any research that that's a good idea. Is there a way you could possibly get this person either in to see you every week? Or if they literally can't come in because of money, do the second week by phone? (Transcript 4, p. 37-38)

Correction appeared to function as a form of feedback that kept clinicians adherent to the CPT protocol. The consultants' corrections seemed to reflect that 
they were listening closely to the clinicians, and were able to provide them with individualized feedback accordingly.

Praise. "Praise" refers to an expression of approval or admiration made by the consultant in reference to the clinician's work. These expressions reflected the sentiment "good job" or "nicely done". Although Praise was usually directed toward an individual, it was sometimes directed toward the group. Consultants praised clinicians for the following reasons: enrolling clients; presenting a well-reasoned case formulation; asking good questions; working well with clients; offering an illustrative example of a central concept; identifying key stuck points; adhering to the protocol; and, engaging with the process of consultation. The following statement exemplified a consultant's use of Praise directed toward the group:

Excellent. You guys are so wonderful because you're really getting at the heart of what we need to be addressing, is what's going on in your own minds when you're choosing cases. The fact that you're so willing and open to discuss this and get feedback from me is lovely. (Transcript 4, p. 29-30)

The following excerpt illustrated a consultant's use of Praise directed toward an individual:

Consultant: It sounds like she's looking a whole lot different. She's doing a lot less "should, should" and she's allowing some of that natural emotion to dissipate.

Clinician: Yeah, so it seems like she's on the right track, so...

Consultant: Yeah, I think that's fantastic. So, yay! Yay!

Clinician: Yeah.

Consultant: Yeah, I mean I think that's amazing. I'm super happy for her and for you because you get to see this work. (Transcript 13, p. 5) 
Praise was used to highlight what a clinician was doing well. The act of praising something seemed to draw attention to it, and signal its importance. Through praising a specific behaviour, the consultant likely sent a message to all the clinicians that a particular behaviour was desirable or valuable. Praise may have indicated to clinicians what to pay attention to in session, thereby improving their fidelity to protocol. Praise may have also functioned as positive reinforcement - by identifying what clinicians were doing well, they may have been more likely to engage in that behaviour in the future. The use of praise was thought to signal to the clinician that he or she was on track, which may have affirmed his or her sense of mastery over the protocol. This may have contributed to the clinician's sense of confidence, in turn increasing his or her engagement and motivation. The feelings of warmth generated by praise may have increased the sense of connectedness between the consultant and the clinician. It is thought that the Provision of Feedback contributed most directly to clinicians becoming proficient in delivering CPT.

\section{Mechanisms}

\section{Reflection}

Reflection is posited to be a mechanism of consultation. "Asking if Clinicians Have Questions" and "Inviting Participation or Reflection" are elements of consultation that encourage clinicians to reflect.

Asking if Clinicians Have Questions. This element refers to an instance whereby a consultant asks the clinicians if they have any questions. Sometimes the solicitation of questions was in reference to something very specific (e.g., any 
questions about the trauma account; how to use a particular worksheet), while other times it was more general. The consultants' statements, "Any other issues come up for you in session one or two that would be helpful for me to review?" (Transcript 1, p. 4), and “Anybody have questions about that person?” (Transcript 10, p. 14) both exemplified this hypothesized mechanism of consultation.

Asking clinicians if they had questions appeared to function to foster a culture of curiosity, inquiry, and reflection. The frequent invitation to ask questions seemed to normalize the experience of not knowing, and encouraged clinicians to seek clarification as needed. Additionally, by encouraging clinicians to ask questions, the consultants gave the clinicians the opportunity to guide their learning process by determining the content of the calls. This likely reflected the consultants' commitment to facilitating clinician learning by tailoring the calls to meet their needs. Through these statements, the consultants may have sent the clinicians the message that they should be reflecting on what occurs in session, and they regarded the act of asking questions as a valued and necessary component of learning. When consultants asked whether the clinicians had questions about something specific (e.g., the content of a particular session) it seemed to validate that the topic may not have been straightforward, and that they should seek clarification as needed.

Inviting Participation or Reflection. "Inviting Participation or Reflection" refers to instances of consultants inviting clinicians to participate in the group discussion or reflect on a question raised by another clinician. Typically, it came in the form of asking the clinicians if they had thoughts they wanted to to share about another clinician's case, or in response to another clinician's question. The essence 
of this element was the consultant asking the clinicians to share their thoughts or ideas on the issue at hand. In the statement, "What can avoidance look like? And, I want everyone to jump in on this. What does avoidance look like?" (Transcript 4, p. 25), the consultant's encouraging all participants to participate illustrated this element of consultation. Additionally, the consultant's statement, "[Name X], did you have any thoughts that you wanted to add into this conversation about [Name Y's] case?" (Transcript 11, p. 12) also exemplified this element of consultation. Inviting participation or reflection seemed to function to urge clinicians to think deeply about the various questions and issues discussed during consultation. By encouraging clinicians to reflect, consultants appeared to foster an environment of active learning that supported both knowledge consolidation and case conceptualization. Creating an environment in which participation was valued may have also helped clinicians remain engaged in the consultation process. By inviting clinicians to participate and reflect, the consultants seemed to slow down the pace of the discussion, encouraging clinicians to engage in a more active form of learning, and sending the message that critical thinking is an important component of learning. When several clinicians shared their thoughts on one topic, it seemed to facilitate creative problem solving. Additionally, when a clinician shared his or her thoughts on another clinician's case, it may have benefited the responding clinician's learning - he or she may have been struggling with a similar issue with regard to one of his or her own cases, and reflecting on it to assist with a colleague's case may have in turn helped with his or her own. 


\section{Connectedness}

Connectedness is posited to be a mechanism of consultation.

"Encouragement" and "Validation" are elements of consultation that foster connectedness.

Encouragement. "Encouragement" refers either to statements made by consultants that serve to make the clinicians feel confident about the therapy going forward, or about the progress they can expect to make with their learning. Encouragement captured statements that were future-oriented. Typically, Encouragement was offered around the process of learning the therapy, understanding the therapy, and clarifying the therapy's terminology. The most frequent form of Encouragement was letting clinicians know that delivering CPT would get easier the more comfortable they became with the protocol. The following examples illustrated Encouragement:

I think that is a goal that you want to do both. But there are ways to not feel stressed by staying on protocol. It will become and feel much more natural as you get more comfortable with it. (Transcript 6, p. 21)

I definitely think, [Name X], that you've got a good understanding, and the fact that we're having this kind of discussion at this point, this is just coming up with additional ideas, but I do get the sense and I think with more sessions like you said, all the nuts and bolts will become more second nature. (Transcript 27, p. 20)

By reminding the clinicians that delivering CPT would get easier, the use of encouragement seemed to function to keep their morale high, and motivate them when the learning was difficult. These supportive statements may have prevented the clinicians from becoming discouraged, and helped them remain engaged and motivated. 
Validation. "Validation" refers to instances where the consultant confirmed a clinician's perspective, decisions, or experience. In the statement, the consultant took the perspective that the clinician's thinking was reasonable, or his or her course or action was understandable. Consultants validated the following: how difficult it can be to listen to a client describe a traumatic event that is especially horrific; challenging or frustrating interactions with clients; the challenges associated with certain types of clients (e.g., highly dissociative clients); and, the challenge of learning to deliver CPT. The consultant's statement, “I mean, I can understand that, because I know we talked a lot about trying to format those stuck points and that can be time consuming," (Transcript 3, p. 6) exemplified the use of Validation. The following statement also illustrated this element of consultation:

I hear you, and that's the tricky part about the index event. I hear what you're saying, and you don't want to be in the position that you're drawing the line in the sand at session one and he's clearly saying, "This is what I want to work on," and you're clearly saying, "I don't really care what you want to work on, this is what we're working on". You don't want that perception to come across at all, so I hear what you're saying because that would be very invalidating, and the antithesis of empowerment that we see with these clients in CPT. (Transcript 2, p. 9)

Validation may have functioned to normalize clinicians' experiences, which in turn may have helped to reduce their anxiety around the learning process. With reduced anxiety, clinicians may have been able to problem solve more effectively. Additionally, knowing that others have struggled to learn the therapy, especially the consultants who are likely to be perceived as knowledgeable and competent, may have functioned to reassure the clinicians of their own capacity to deliver CPT proficiently. It may also have helped the clinicians appraise accurately the learning process by making it seem like a predictable experience, contributing to the 
clinicians' perceptions that it is something they will be able to learn as well. This may have served to enhance the clinicians' engagement and motivation. Additionally, by sharing their own experiences with the clinicians, the consultants may have been perceived as authentic, which may have contributed to feelings of connectedness between the consultant and clinician.

\section{Objective 1b}

The results presented above described what are posited to be the primary elements of clinical consultation. They also included a description of contextual factors that are thought to influence consultation, and the hypothesized mechanisms of consultation. The results presented below describe how all of these elements are thought to function together, and how consultation is posited to work (see Figure 2). The function of the elements of consultation that comprise the theme Organization (i.e., Agenda Setting, Attendance, and Structure) is thought to be to create an environment in which learning can occur. The excerpts below illustrated how Organization seemed to create this environment. The following excerpt exemplified Attendance, and was extracted from the very beginning of a call:

Consultant: Hi everybody, it's [Name W].

Clinician X: Hi.

Clinician Y: Hello [Name W].

Consultant: All right, I see we've got um, [Name X] and [Name Y] and not [Name Z] yet, um, so, maybe she'll join us in a few minutes? (Transcript 24, p. 1) 
Once the consultant established who was on the call, she encouraged the clinicians to begin the call by determining who would go first. The following excerpt exemplified Structure:

Consultant: But we can go ahead and get started. How are y'all doing?

Clinician Y: Good, good.

Clinician X: Good.

Consultant: All right, so who would like to get started?

Clinician Y: Want to go ahead, [Name X]?

Clinician X: Um, uh okay, sure! (Transcript 24, p. 1)

The following statement made by the consultant exemplified Attendance:

I guess [Name W] should be on today, so maybe she'll join us in a few minutes. But, like I said [Name X] is not going to be on, so [Name Y], [Name Z]. (Transcript 29, p. 1) 


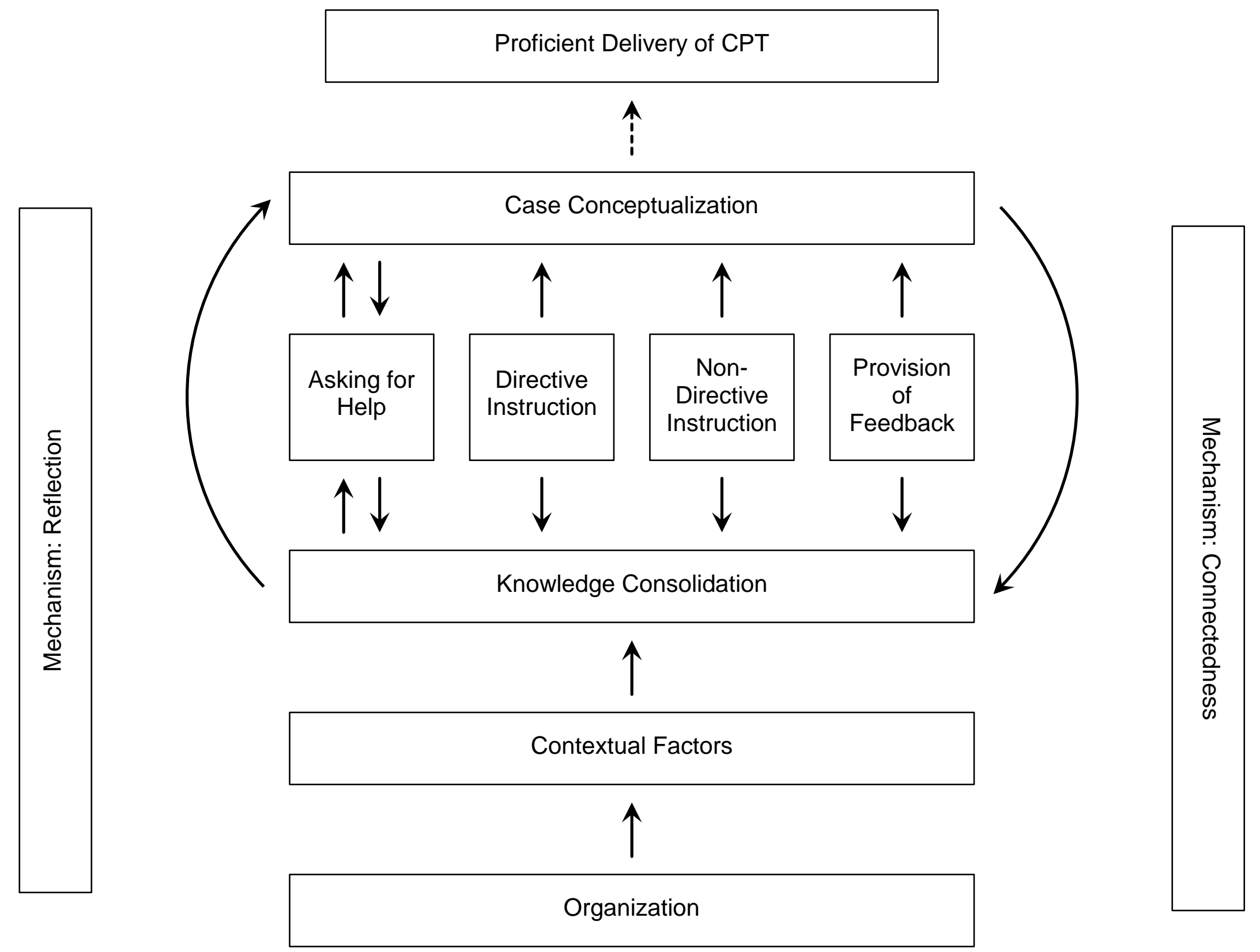

Figure 2. Model of consultation 
The statement was immediately followed by an exchange that captured Structure:

Consultant: Which one of y'all wants to go first?

Clinician Y: You go, [Name Z]

Clinician Z: Okay, right on. (Transcript 29, p. 1)

In the following excerpt, after both clinicians articulated their needs for the call, the consultant summarized this information by setting an agenda. The following statement made by the consultant exemplified Agenda Setting:

Looking over kind of, so [Name Z] might share a little bit more about that final impact statement, and then [Name Y] we're going to talk about your 46year-old, and then [Name W] your ... (Transcript 29, p. 4)

The presence or absence of various contextual factors seemed to serve to either facilitate or hinder learning. For example, when technology functioned well, the call ran smoothly and the impact of technology did not seem to be at the forefront of participants' experience of consultation; however, when technological disruptions occurred, they seemed to interfere with learning. Oftentimes, technological disruptions were minor and resolved easily. The following excerpt illustrated a minor technological disruption:

Clinician Y: Just a sec. I'm going to try another phone. Can you hear me better on this phone?

Consultant: Are you guys hearing well?

Clinician Z: I'm hearing a little better. Ok, it might just be that phone. (Transcript 5, p. 12)

Occasionally, technological issues were major and seemed to interfere significantly with clinician learning. For example, in the following excerpt, the 
clinician expressed that she missed a considerable portion of the call because she faced significant difficulties connecting.

Consultant: Okay, excellent and [Name of Clinician] have you been struggling to get on?

Clinician: I have been struggling for, well it took me about 35 minutes to connect today.

Consultant: I'm so sorry - that's an inordinate amount of time. (Transcript 20, p. 13)

Another contextual factor that seemed to affect the call was whether all the clinicians were present at the beginning of the call, or joined late. When all the clinicians were present at the outset, they all received the same dose of consultation and had an equal opportunity for learning. When clinicians joined late, they disrupted the flow of the call, and missed the opportunity for learning that came with the content that had already been discussed. In the following example, which begins midway through the call as the consultant was providing an explanation, two clinicians joined late and disrupted the flow of the call. The consultant responded by briefly orienting them to the discussion, and then assuring them they would be able to add their items to the agenda shortly.

Consultant: ...So that's why we focus on that first. We want to know what they're saying that's adding an extra load of pain emotionally. It might be coming from the interpretations and then we want to help them to grapple with the actual full story, the factual story, which may change their interpretation when it has to do with fault. So two things that he's-

Clinician X: [Name Y] and [Name Z].

Clinician Y: Okay, hi guys this is [Name Y].

Clinician X: Hi. 
Consultant: Hi, we're just getting starting...well not just getting started, we actually we did a little bit of a checking in and now we're talking about [Name $X$ 's] case and we're looking for assimilation from his patient's impact statement. So if you would, I think we'll continue that conversation and then we'll check in with you [Name Y] and [Name Z]. (Transcript 18, p. 6)

In the following example, the clinician missed the case conceptualization, and the opportunity for learning that it provided, which occurred prior to her joining the call.

Consultant: It's interesting. I think that makes sense. That sounds like it was a belief that was born during a chaotic dysfunctional and abusive environment at an important developmental time.

\section{(Transcriptionist's note: New clinician joins the meeting.)}

Consultant: It sounds like this predated an adult trauma and it was born, she heard it loud and clear, from her family of origin. (Transcript 15, p. 3)

Since consultation seems to be largely determined by clinicians' questions, whether or not clinicians came to consultation prepared with cases to discuss is thought to be the contextual factor that had the greatest influence on consultation. Clinicians may have felt demoralized when they did not have case material to present, which may have led to them disengaging from the learning. In the following statement, the clinician expressed regret that he did not have case material to present.

Clinician: I wish I had something to offer. I, in fact, I have I have, you know I have people both for intake on the outpatient unit who, they're looking for people with trauma that I can treat here and I've even asked the military for any cases, and as of yet, I don't have any. (Transcript 3, p. 3)

The excerpt that follows exemplified the way in which learning seemed to be negatively affected by clinicians who did not come prepared with questions or cases to discuss. 
Clinician: I was just saying that I haven't seen my person since last Tuesday, and I'm seeing her this afternoon for session number eight, so I haven't done session number eight yet with her. And, I have a couple of people coming in, within this next week, that I'm hoping may be candidates to be my second person, but I haven't actually done another session since I talked to you last, so I don't really have much more.

Consultant: So, you don't have any questions, probably, at this point, is what you're saying?

Clinician: No. Right. (Transcript 11, p. 1)

Access to individual support occurred when so many clinicians were absent from a call that the sole clinician present received individual consultation. Although this likely hindered learning in several respects, it may have enhanced learning in others. The absence of a group dynamic meant that clinicians could not learn from each other's cases. The clinicians who were absent did not have the benefit of hearing the material discussed on the call, and the clinician presenting his or her case did not have the benefit of hearing the perspectives of the other group members. Additionally, when clinicians were alone on the call they may have felt disconnected from the clinicians who were absent. The following statement, made by a consultant, reflected her concern that the lack of a group experience would negatively affect learning:

I wonder where everybody else is today. We had [Name X] on last time, and we had [Name Y]. There's supposed to be a few other people. One of them I knew was going to be absent today. You know, it's okay if we are a little bit rocky getting started, but my only concern is then it just doesn't seem like you guys get the benefit of the consultation if we're ending up not being able to have cases 'cus it's a dead call for you guys, so I feel badly about that. (Transcript 3, p. 4) 
At the same time, it is possible that being the sole clinician on the call provided a unique opportunity for that clinician to receive tailored and thorough feedback.

The presence or absence of each of these contextual factors may have affected the success of a particular consultation call, in turn contributing to the extent to which clinicians were able to make use of the consultation process to achieve proficiency. Under optimal circumstances, technology functioned well, the call was well attended, the clinicians joined the call on time, and the clinicians came prepared with questions to ask or case material to discuss.

It is posited that an overarching function of consultation is knowledge consolidation. A thorough understanding of both the therapy's underlying theory and content are thought to be the foundation upon which clinicians deliver treatment and conceptualize cases. Knowledge consolidation is thought to occur primarily through information-based teaching. The content of information-based teaching was determined primarily by clinicians' questions. The following excerpt exemplified a clinician Asking a Factual Question, and the consultant's reply exemplified Information-Based Teaching:

Clinician: ... I'm just wondering if you can remind me what a stuck point would sound like if it was an assimilated one versus an ${ }^{1}$ accommodated one.

Consultant: ...So assimilated, when we talk about it in the training we talk about assimilated are usually looking in the past, like looking back and they usually are things like self-blame, so "I should have done this" and it usually

\footnotetext{
${ }^{1}$ Note: This clinician has used the term "accommodated" when the correct term is "overaccommodated". In the consultant's reply, she uses the term "over-accommodated".
} 
results in a lot of guilt or shame. They can also be undoing. Undoing is where you kind of try to somehow take it back. So like for assault it could look like, "I guess he didn't understand that I didn't want it, so it wasn't really alright." I've even seen people try to undo by saying things like, "I just shouldn't have reacted this strongly. I'm in war, so I know bad things happen, so I shouldn't be having this reaction. It should not have upset me". Assimilating can also be other-blame, which is when you kind of inappropriately blame somebody else. So, "My commanding officer should have done something different, or was lazy and didn't care and that's why this happened". So, it can be, sometimes other people like the perpetrator do have responsibility and are to blame, but when people are inappropriately assigning blame. So like, "My parents should have protected me from the abuse," but maybe the parents didn't know. So that kind of thing. So that's all assimilated. Basically, looking back and trying to change something about what happened, or about how you see the world in order to make sense of it. Over-accommodated is when you're kind of looking in the present and future. So, now this has happened to me, now that I was assaulted, or I saw this death, or I experienced this event now I know from here going forward that I'm a bad person, so I can never trust anyone. I'll never be safe no matter where I go - that the world is just out to get me. Okay, so these over-accommodated it's like now I have changed my belief system. I've recognized that I was traumatized, but now I've gone overboard in looking at how I'm going to view the rest of the world and myself in light of the traumatic event. So that's over-accommodated, and our goal is to get to that balanced belief of accommodation, which is the inbetween where you look at, okay this is what happened, this is a balanced view of what happened, I'm taking responsibility where I need to but I'm not taking too much, I'm putting it where I need to and I have a balanced view of, "Yes, there is evil but not everyone is evil." (Transcript 1, p. 12-13)

On the same call, after the clinician presented her case and the group discussed its conceptualization, the consultant said, "By the way, her assimilated stuck point includes that the operator didn't care - that's an assimilated stuck point - and that the police were annoyed - that's an assimilated stuck point" (Transcript 1, p. 14). In this way, asking factual questions, reviewing information about the protocol, and case conceptualization are linked, and all function in the service of knowledge consolidation. The consultant used the clinician's case material to illustrate further what an assimilated stuck point was. 
Along with asking a factual question and information-based teaching, case conceptualization seemed to further facilitate the process of knowledge consolidation. Case conceptualization often provided useful and concrete examples that illustrated the concepts clinicians were seeking to clarify. In this way, case conceptualization contributed to knowledge consolidation. At the same time, clarifying and deepening their understanding of central concepts through information-based teaching seemed to assist clinicians with case conceptualization.

Like knowledge consolidation, case conceptualization is posited to be an overarching function of consultation. Case conceptualization consisted of formulating the client's problems according to the theoretical framework that underpins the therapy, and determining how to intervene by targeting the hypothesized mechanisms of change. Engaging in case conceptualization seemed to reinforce knowledge of the protocol. For example, in the following quotation from a clinician, which exemplified Case Conceptualization, she used several key terms associated with therapy (i.e., "impact statement", "stuck points", "impact statement", "ABC sheets").

Clinician: Definitely in sessions two and when he was reading his impact statement and this last session we did discuss some of his emotional response and reaction. I don't know if it's hard to say, but even though the PCL score doesn't seem to indicate intense symptoms he definitely feels like he has PTSD. It's definitely present in the room. (Transcriptionist's note: Consultant asks clinician to provide background information about the client). Say a little bit about his situation, he was in Afghanistan. He came home in September of 2012 and the key events were around IEDs. Well actually there was sort of involving children. He was involved in picking up body parts and things like that and another incident was he had to take out an insurgent with his vehicle he was driving in a convoy and so he had some good stuck points he developed right off in session one which were, "I always have to be prepared in situations because something might go wrong," [and] "People can't be trusted." In session two after he had written his impact statement, 
also done some stuck points around that and they are things like "Other people can't be trusted," "People in authority always abuse their power," "I should be able to protect others," "It's ideal to have several contingency plans," "I'm not deserving of time for myself," "I can't trust human command especially the new chain of command." So those are some of the stuck points we are working on right now. He got talking about him writing trauma accounts. I brought up, it appeared like emotions from him in sessions two and three. So we did ABC, we did actually two ABC sheets - one on (inaudible) therapy and some o[n] the thoughts, "I'll be out of control. I won't be able to control it. It'll be embarrassing." Just off the top of my head right now cuz I don't have the ABC sheet with me. And then we did another one on writing the traumatic account as well and it was the same thing, "I won't be able to (inaudible) anyone there to support me." Those were some of the beliefs that we (inaudible) and he did a really good job. He's actually really on top of his ability to kind of challenge things and do his homework, so that's really good. So, yeah that's where I left him and we discussed him doing it. Actually today he's going to write his trauma account. I see him next Tuesday so. (Transcript 1, p. 6)

Articulating what was done in session, using the protocol's terminology, and identifying examples from their clinical work all seemed to help clinicians consolidate their knowledge of the therapy.

Knowledge consolidation and case conceptualization are thought to be essential functions of consultation, and their relationship seemed to be bidirectional - as case conceptualization reinforced knowledge of the protocol, deepened knowledge of the protocol facilitated accurate case conceptualization. In particular, it is posited that as clinicians deepened their understanding of the therapy's components and its underlying theory, they were able to conceptualize their cases in an increasingly accurate and nuanced way. For example, the clinician's statement, "My client's PCL - there's a heavy matter of avoidance" (Transcript 2, p. 7), reflects her attunement to the role of avoidance as a maintaining factor in PTSD.

In the excerpt that follows, which exemplified Case Conceptualization, the clinician's efforts to conceptualize her case reflects the centrality of identifying 
"stuck points" when delivering CPT. The consultant's attention to these stuck points, as well as the explicit connection that was drawn between the stuck points and the client's emotions seemed to reinforce the clinician's case conceptualization skills and understanding of the therapy.

Clinician: ...It's been pretty hard, trying to help him look at ways that he thinks that are blocking his recovery. But this last session, he did much better because he came in reading his account and really letting himself feel a lot more of the distress about it. And there is still a lot of self-blame and guilt. So, things like 'I should have done something different', 'I should have stopped it', 'all authority can't be trusted', 'nobody is going to help', 'if I had done the right thing, I wouldn't be getting punished by bad dreams and feeling bad now so I must have done something wrong'. And then, he also has this belief that if he had just gone in and shot all of the allied troops that were raping him that, somehow, the world would have realized that. And this country ... this is an acceptable practice. And if he could just get the world to realize that, then children wouldn't get hurt and we wouldn't send troops to Afghanistan. So, those are the key stuck beliefs, right now, that I am working with him with.

Consultant: So, those kinds of beliefs, would you mind re-stating the last two really quick? I was writing them down. I want to make sure I got them correct.

Clinician: So, clearly, there is self-blame and guilt. And I think that is shifting because he is getting in that context.

Consultant: So, what are the stuck points about blame?

Clinician: "If I had done something different and stopped it, the child wouldn't have been hurt," "I should have gone (inaudible)". So, his sense is that a child got hurt because he failed to act.

Consultant: So, there are the two ends. So, there is the self-blame that can come from 'I should have done something different'.

Clinician: Yes.

Consultant: And then, it can translate to 'it's my fault this happened'. Now, does he believe it's his fault this happened?

Clinician: He doesn't believe it's his fault that it happened. 
Consultant: But he does think he should have done something different?

Clinician: He thinks that he should have done something different. (Transcript 6, p. 12)

The overarching functions of consultation, knowledge consolidation and case conceptualization, are posited to be supported through several elements of consultation that have been categorized as follows: Asking for Help, Directive Instruction, Non-Directive Instruction, and Provision of Feedback. Often times, one or more of the elements played a supportive role in ensuring knowledge consolidation or case conceptualization. In some ways, these four categories of elements can be viewed as the tools employed by either clinicians or consultants to support knowledge consolidation and case conceptualization. There was considerable flexibility in the order in which the tools were used. This variability in sequencing is reflected visually in the model of consultation (see Figure 2).

During consultation calls, engaging in case conceptualization often led clinicians to identify and articulate what they needed help with. For this reason, case conceptualization often preceded asking for help. The following excerpt included the end of a clinician's Case Conceptualization followed by her Asking for Help:

[CASE CONCEPTUALIZATION]...She came about ten or so minutes late, so the session was only about forty minutes, but I did get her to read it and we started to identify some points in the trauma account. Um, but part of her story was that when she, right after the situation she found that, like when she called the operator they were really cold and distant to her, so she felt like you know that they weren't really caring about her and then when the police came and did the investigation she felt like they were really cold, annoyed and irritated with her. So, at the end of that session I really just kind of turned everything off and just spent a bit of time just reconnecting with her just to you know let her know that there's support for her and kind of get that connection just to confirm that and [ASKING FOR HELP] maybe you have some ideas too [Name of Consultant] on like connecting at this point on the learning curve and with working with the model and probably a little bit 
maybe (inaudible) you know kind of just trying to make sure I tick the boxes and follow the steps. So, any feedback on that and I also thought about, like this is a client that I have been seeing before and I already have a - she already has a therapeutic connection to me, but just the strength of that avoidance, I really thought, "Wow this is a client that already has some therapeutic connection to me. What if this was somebody I'd just seen?" and this is like the third time I've actually, or fourth time, like that avoidance was really strong so even some tips on maybe how to work on that better. (Transcript 1, p. 2)

Similar to the segment above, in which the clinician's request for help was preceded by the relaying of relevant information about the case, the example below illustrated further that case conceptualization is often followed by asking for help. In this interaction, the clinician updated the group members on what transpired in the most recent session, and then asked for advice about how to engage in Socratic questioning with the client:

Clinician: [CASE CONCEPTUALIZATION] ... I'd say that one of the main positives is that this patient has been really very good at following through, this session, number three, he turned in all six ABC sheets. He really has nailed down the distinction between thoughts and feelings - I didn't have to deal with anything. There was only one correction, in terms of the columns, where he had under column A, something that should have been under B, but other than that. He got the $\mathrm{ABC}$ distinctions down perfectly, he turned in six sheets, [and] two of them were on the trauma. His PCL scores are not changing one iota, - they vary between about 75 to 80 , but I guess I wouldn't expect them to come down by this point.

Consultant: That's right.

Clinician: He does obviously need a lot of work on the bottom two questions of the $\mathrm{ABC}$ sheet. So I guess the good news is I do not really have to do any work in teaching him the distinction between thoughts and feelings, which is a pleasant surprise. [ASKING FOR HELP] What I'm finding, though, is that I guess I need some assistance or suggestions on Socratic questioning because internally, I find myself getting sucked in and sharing his overaccommodations. He is so articulate in what he is writing down, that I'm kind of sharing the over-accommodations with him. So I'm struggling to get in this Socratic questioning because when I read what he has down, some of the time I'm going, internally, yeah, I guess I would think the same thing. (Transcript 10, p. 2-3) 
Another common combination of tools is information-based teaching and giving advice, which functioned together to help clinicians consolidate knowledge. In the excerpt below, the consultant engaged in Information-Based Teaching by reviewing the purpose of the impact statement, and then Giving Advice about how to use the impact statement most effectively when working with the client. Together, the use of these tools seemed to facilitate knowledge consolidation.

[INFORMATION-BASED TEACHING]: The way that we talk about, in the impact statement, your beliefs changing, we talk about before and after the traumatic event. So before you thought this way, after you thought that way. Well, when you're an infant or a baby, you don't have schemas. You have maybe a couple of schemas but you don't have a lot. [GIVING ADVICE]: And so one of the things you might want to think about, as you're working with her, is instead of saying how are your beliefs changed or your belief about yourself changed, is you can say how are your beliefs about yourself and others in the world formed because of this experience. And so how did she build her understanding of this. And then, retrospectively, what did she tell herself about how something like this could happen. What you're probably going to get is, instead of self-blame, you're going to get a great deal of otherblame, of questioning the goodness of the world. You can still work on that. And then the problem is that it's going to have been confirmed with some other traumatic events. And so what you're going to want to help her do is realize that she also may be kind of, what I call connecting the dots, which is this bad thing happened, this bad thing happened, this bad thing happened, therefore the world is bad. But then you miss all the other data that said the world was good because you only connected the negative events to come to your conclusion, versus taking in all the data. So you've connected the dots between just the bad things and then got your result. So that's conceptually how I would anticipate you're going to need to work with her. (Transcript 7, p. 16-17)

Although giving advice and information-based teaching were commonly used together to facilitate knowledge consolidation, the order in which they were used varied. Unlike the example above, in which Information-Based Teaching preceded Giving Advice, in the following example, Giving Advice preceded Information-Based

Teaching. Specifically, the consultant gave the clinician advice about how to 
approach a particular situation, and then reviewed content that was covered in the workshop:

[GIVING ADVICE] Use it as an opportunity to address your stuck point. Try to understand what the nature of the emotion is that the person is feeling in the nightmare because, maybe, you can teach them about the kind of emotions they are having, perhaps, that they are worried about having or acknowledging that they are having with regard to this event. And then, I'll add just one more thing on this, the images in the event. The images in the event can give you some clues about aspects of the trauma that are particularly troubling, traumatizing that, in the light of day (inaudible) to talk through as they are focusing on. So, both might need some processing of natural emotions but also, maybe, some (inaudible)...[INFORMATION-BASED TEACHING] But that's precisely one of the reasons why we have these strategies that we teach and practice before we do this trauma processing. Do you remember that in your training? They did that biological model and they showed that little picture of the brain - that very abstract depiction of the brain and the cognitive therapy approach about re-gauging their cortical brain areas and keep them awake and active when they talk about the trauma. Because, in PTSD, what happens often is you - at least, what the biology tells us is that stress hormone deregulation (inaudible) cells in the cortical brain area lose their ability to function. And in adapting, the emotions just run amuck. They get to rule the roost. (Transcript 12, p. 18)

In addition to the commonly occurring combination of information-based teaching and giving advice, several elements of consultation were frequently used alongside case conceptualization. Specifically, Directive Instruction, Non-Directive Instruction, and the Provision of Feedback were frequently used to enhance case conceptualization. In the following example, the consultant offered advice regarding what to focus on in response to the clinician's relaying of the client's stuck points and what they covered in session:

Clinician: ...[CASE CONCEPTUALIZATION] in session three you introduce the $\mathrm{ABC}$ worksheets and I try to introduce those worksheets to work with some of what I perceive to be stuck points around his mother's death, but that's where I say on the fly I did decide okay assimilated versus accommodating. What I do know clearly is that he feels it's his fault that she died and that he can't be trusted to make (inaudible). I mean, I was able to challenge some of them like, "I cannot be trusted to make the right decision." I was able to 
challenge that one to some degree but he goes, "No it was my fault that she died." So, I kind of hope to take a shot working on these, and also to buy myself some time to talk to you today.

Consultant: Sure, and I think that you can definitely have time to get to both of them. So, [GIVING ADVICE] I would stick with the idea that you really do want to focus in a primary session on the index trauma, and the index trauma is the one that is the most closely linked to the PTSD. (Transcript 20, p. 11)

In the following example, the consultant employed a Correction in response

to something she heard the clinician relay when the clinician described what

occurred in the most recent session:

Clinician: [CASE CONCEPTUALIZATION] This just happened recently - he actually didn't do the homework, hadn't read the materials; my sense was he really didn't understand, and I didn't pick that up at the end of the session that he really was not comprehending what the next part was about. It was on the safety module. So I felt I needed to go back to that and review the content about safety and reassign that. I did try to work with him in the session to kind of walk through...

Consultant: Reassign what, the module?

Clinician: Yeah, the module. I did try to walk through what he should have done in the homework, in terms of picking a safety issue to work on a challenging beliefs worksheet with. He hadn't done any of those; he'd been away on a camping trip with other people from his OSI support group. I tried to work through a safety issue with him and it felt to me like he really didn't have the concept, so I spent that session -

Consultant: The concept of safety, or the concept of the challenging belief worksheet?

Clinician: Actually, both. He's still struggling a bit with the whole worksheet, but he's much improved on that. In terms of safety, for self and for others, it may be a function of he gets quite inflexible in his thinking so he grabs on to an issue and that's where he goes. Actually, he was more in trust. I said, oh, this is the trust module here.

Consultant: Let me back up a little bit and explain the last five sessions. [CORRECTION] I would actually encourage you not to, at any point in the protocol, but particularly at the end, there isn't necessarily, given how it can work, a reason to repeat a session. In fact, whenever you break the protocol, which repeating a session is considered breaking the protocol, because it's 
not built in to repeat them, per say, one of the costs of breaking that protocol is that you lose that benefit of the structure that keeps them moving forward and is always working against that avoidance. (Transcript 14, p. 4-5)

In the following example, the consultant praised the clinician and then gave her advice about how to proceed.

[PRAISE] That's really wonderful. That's nice (inaudible). So, it sounds like you have a really solid grasp of how to address and deal with these modules. [GIVING ADVICE] I might ask him if he feels that the reading, and the reassignment of the reading of the materials, the patient materials as part of the protocol is burdensome or beneficial. Explore it and encourage him to read it. But if you are covering the materials satisfactorily, and it sounds like you are, in the session, then I wouldn't dwell on it. (Transcript 12, p. 25)

Finally, an essential component of the model of consultation is thought to be its two hypothesized mechanisms: reflection and connectedness. These mechanisms are processes that seem to operate at numerous points during consultation and facilitate the overarching functions of consultation, namely knowledge consolidation and case conceptualization. Some elements of consultation may be especially likely to foster reflection. Consultants seem to foster a culture that supports reflection when they employ the following elements of consultation: Asking if Clinicians Have Questions (e.g., "Then what might be your questions or issues that you'd like to discuss this session?" [Transcript 16, p. 4]); Inviting Participation or Reflection (e.g., "What might you do, and how much more would you do to get the patient in touch with her feelings? Either one, [Name X] or [Name Y]" [Transcript 16, p. 7]); and Socratic Questioning (e.g., "How could you check out and find out if there's a stuck point and what that stuck point it? How would you do that?" [Transcript 16, p. 7]). Additionally, consultants seemed to encourage clinicians to reflect when they shared a personal experience. For example, in the 
following excerpt, the clinician's response to the consultant's Sharing a Personal Experience suggested she was reflecting on what the consultant had shared:

Consultant: Sometimes I've had folks have ideas that put them at first, they think "Oh hell, it's going to happen anyways, it doesn't make a difference what I do."

Clinician: And isn't that about regulating the fear though that they can't...like isn't that sort of one of the distractions from either the fear or something else possibly? (Transcript 9, p. 7)

Finally, Asking a Factual Question (e.g., "Would it be outside the model to have her do some breathing exercises?" [Transcript 11, p. 20]), and Asking for Help (e.g., "I'd like your thoughts on that" [Transcript 20, p. 14]) are both elements of consultation that seemed to exemplify that clinicians were engaged in reflection.

Like reflection, connectedness is thought to occur at numerous points during consultation. For example, when consultants imparted information, engaged in collaborative case conceptualization, gave advice, or modeled behaviour, they created opportunities for connectedness. However, some elements of consultation appeared especially likely to generate feelings of connectedness. Consultants seemed to foster connectedness when they employed the following elements of consultation: Encouragement: (e.g., "I definitely think [Name X] that you've got a good understanding...and I think with more sessions, like you said, all the nuts and bolts will become more second nature" [Transcript 27, p. 20]"), and Validation (e.g., "...what you did is fine too, and you do have the best of both worlds because he was able to work on it on his own" [Transcript 29, p. 6]). As well, in the example below the consultant drew on an experience that was familiar to many clinicians. Sharing a 
Personal Experience seemed to foster feelings of connectedness between

consultants and clinicians:

...to me this always reminds me a little bit of when you first ask people about suicidal ideation, and people think, "Oh, I don't want to ask about that because I might create it, you know, they wouldn't have thought of it before, but from my experience people know what they think and what they don't think, and if you think, "Okay I can't trust anyone," me asking you, "Can you trust anyone?" isn't going to make you go, "Well maybe I shouldn't," just like if I didn't think it was my fault, then someone asking me, "Do you think it was your fault?", I'm not going to change my mind just from somebody asking me the question. (Transcript 29, p. 6)

By providing emotional support to clinicians who were learning the therapy, and describing their own experiences delivering the therapy, the consultants created opportunities for closeness with the clinicians. This seemed to foster feelings of connectedness.

\section{Objective 1c}

An additional aim for this study was to identify context-mechanism-outcome (CMO) configurations. In reviewing the transcripts and examining patterns across transcripts, three CMO configurations were identified. The first CMO configuration posits that technological success (Context) facilitates connectedness (Mechanism 2) among clinicians who would otherwise not be able to meet for clinical consultation. Technological success makes consultation possible, and the activity of consultation creates many opportunities for reflection (Mechanism 1). This leads to proficient delivery of the therapy (Outcome; see Figure 3).

The following example was extracted from a consultation call in which there were no technological disruptions (Context). Participation in consultation facilitated 


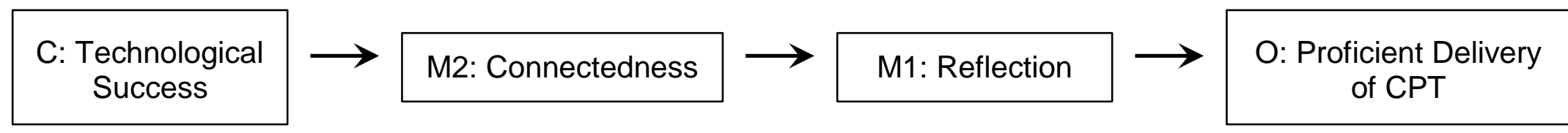

Figure 3. Context - mechanism - outcome (Configuration 1) 
connectedness (Mechanism 2) among clinicians, which generated opportunities for reflection (Mechanism 1). During this consultation call, the consultant fostered many opportunities for reflection (Mechanism 1). For example, the consultant asked, [INVITING PARTICIPATION OR REFLECTION] “Does anyone have any other thoughts in terms of choosing that index event and that kind of switch from what she had originally thought to what she's going with?" (Transcript 8, p. 5), and [SOCRACTIC QUESTIONING] "How would you say that to a client? I mean, in this situation, how would you guide her through that?" (Transcript 8, p. 8), and [ASKING IF CLINICIANS HAVE QUESTIONS] “Any other questions or concerns about session two coming up, or anything else?" (Transcript 8, p. 17).

The second CMO configuration posits that bringing case material (Context) facilitates reflection (Mechanism 1), which helps clinicians consolidate their knowledge, refine their case conceptualization, and develop their skillset. Additionally, sharing one's cases with the group, and the act of giving and receiving input on cases generates feelings of connectedness (Mechanism 2). Together, this contributes to the proficient delivery of the therapy (Outcome; see Figure 4). The following examples were extracted from a consultation call where all of the clinicians brought and presented case material (Context). In the following examples, the consultant simultaneously seemed to foster a culture of reflection (Mechanism 1) and promote feelings of connectedness (Mechanism 2) among the clinicians by encouraging the clinicians to provide input on each other's cases:

[INVITING PARTICIPATION OR REFLECTION] I'm curious what [Name X] and [Name Y] might have to say about if this were your patient, given how you've described the session is and how the patient is, what might you do and how 


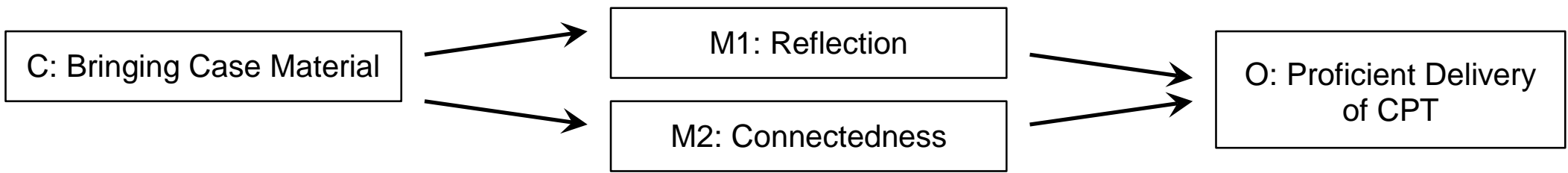

Figure 4. Context - mechanism - outcome (Configuration 2) 
much more would you do to get the patient in touch with her feelings. (Transcript 16, p. 7)

The consultant's harnessing of both reflection (Mechanism 1) and connectedness (Mechanism 2) were illustrated in the following example:

[INVITING PARTICIPATION OR REFLECTION] [Name X] or [Name Y] any thoughts, questions or comments regarding [Name Z] and the work that he did with his patient? And any questions that you might want to ask about dealing with something like this? (Transcript 16, p. 11)

The third CMO configuration posits that clinician punctuality (Context 3 ) maximizes opportunities for clinicians to reflect (Mechanism 1) on the material shared by the other clinicians and to experience feelings of connectedness (Mechanism 2) among themselves and with the consultant. Maximizing one's dose of consultation increases the likelihood of becoming proficient in the delivery of the protocol (Outcome; see Figure 5).

The following excerpt was extracted from a consultation call where all the clinicians were punctual (Context 3). This provided ample opportunities for clinicians to reflect (Mechanism 1). Consultants fostered reflection (Mechanism 1) by asking questions such as [ASKING IF CLINICIANS HAVE QUESTIONS], “So, any questions for the call, [Name X]?" (Transcript 23, p. 2). The consultant also encouraged reflection (Mechanism 1) through her use of Socratic Questioning:

And, what do you think the core beliefs that, that um, she started to alter that were set up by the hazing? Like, how do you, what kind of beliefs was she endorsing from the hazing and all the other things that happened to her? (Transcript 23, p. 4)

In addition to the numerous opportunities for reflection (Mechanism 1) afforded by the clinicians' punctuality (Context 3), it provided ample opportunities to foster feelings of connectedness (Mechanism 2). In the following example, the 

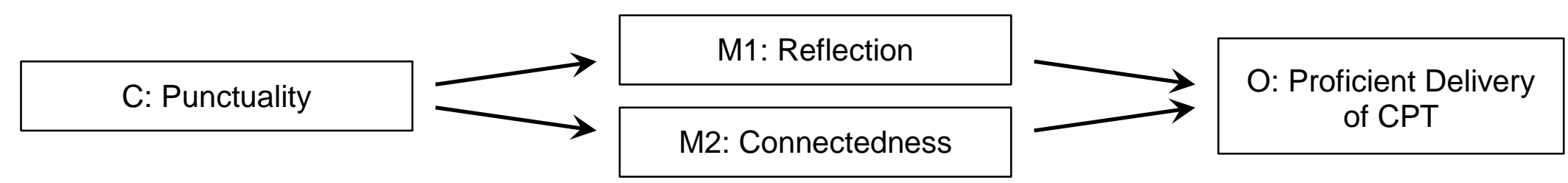

Figure 5. Context - mechanism - outcome (Configuration 3) 
consultant used Validation, which seemed to facilitate feelings of connectedness

between the consultant and clinician. In parallel to the importance of

connectedness in consultation, in the excerpt below the consultant reflected on the

importance of connection as a mechanism of change in therapy:

Consultant: Well, I think that you, I feel a lot of the times that I feel that way as a therapist, it's really a reflection of what's going on with the client. I mean because you're not spinning your wheels with other people so. Um, she's spinning her wheels and you're caught up in it so that's how she feels. So then how do you, how do you, um stop doing that and it feels like, um, what you're telling me what I'm hearing on consultation, is that there's a shallow kind of superficial, feel, to the work that you're doing

Clinician: Yes.

Consultant: And that, that lacks emotion right, emotion is how we build intimacy with each other it's how we feel connected, and so she's avoidant, and, and the emotional piece is what's missing, and I think you're right I think you've got to unlock that in order for there to feel like there's some connection in progress being made. (Transcript 23, p. 11)

See Figure 6 for an overview of how all of the CMO configurations function together.

\section{Objective 2}

The second aim of this study was to compare the model of clinical consultation developed in this dissertation to the model of clinical supervision put forth by Milne and colleagues (2008). Research conducted in both the areas of clinical supervision and consultation has been impeded by poor conceptualization of what these educational processes include and how they work. These models shared the underlying aim of constructing an empirically based model that can be tested.

A key similarity between the model of supervision put forth by Milne and colleagues (2008) and the model of consultation put forth in this dissertation was 


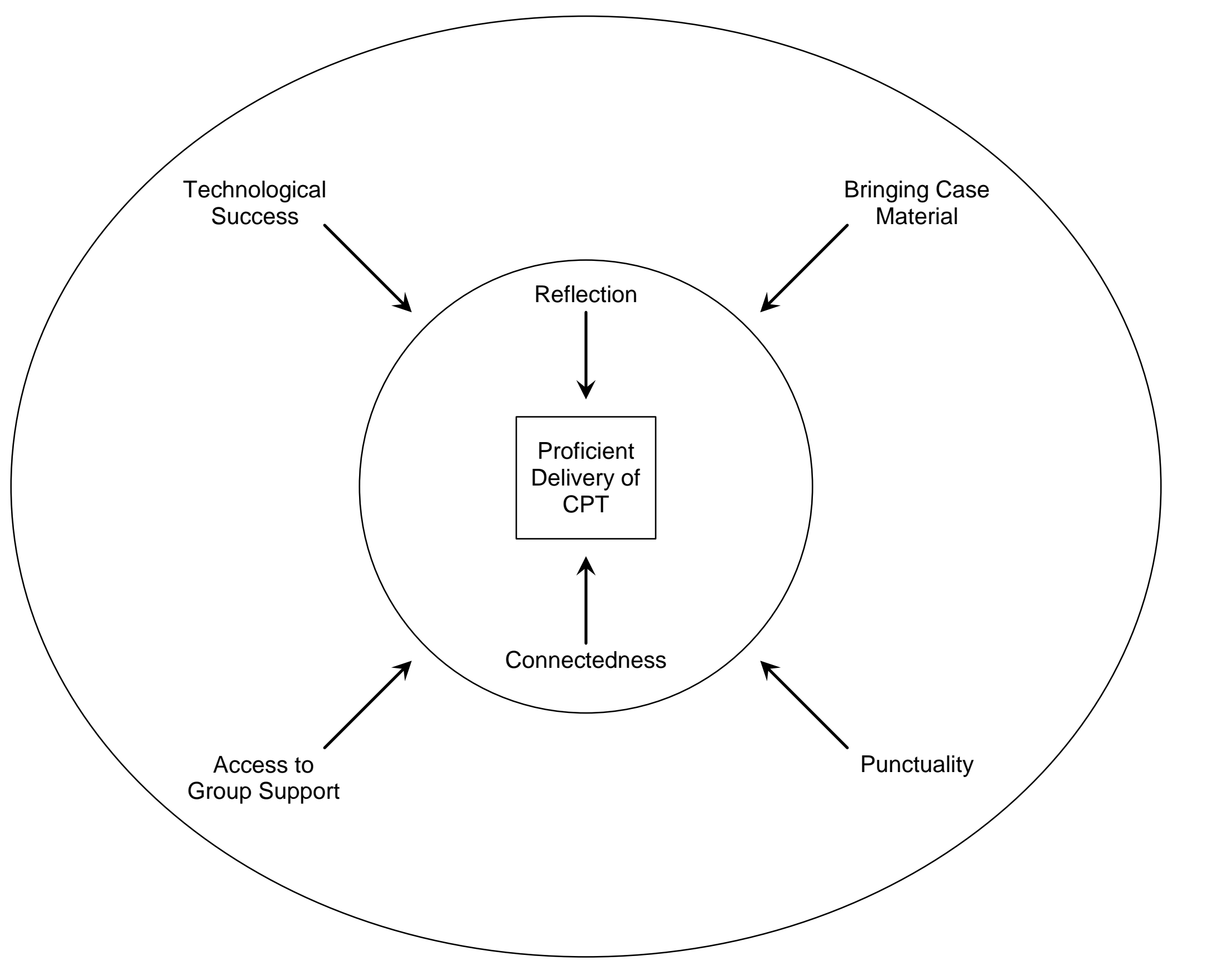

Figure 6. Complete context - mechanism - outcome configuration 
the identification of key contextual factors that are posited to facilitate and hinder the learning process. Both studies highlighted the importance of considering contextual factors, and of identifying factors that seem to contribute positively to learning and those which contribute negatively to learning. With regard to contextual factors, administrative support was the most frequently mentioned contextual factor in studies about supervision, and it was thought to have a positive influence on supervision. Overall, the contextual factors identified in both models facilitated a learning context.

An additional similarity between the two models was the identification of the primary elements that comprise the activity of interest. With regard to the "supervision interventions, ${ }^{2}$ " as they were called in the study by Milne and colleagues (2008), and the "elements of consultation," as they were called in the present study, several of the most common were shared. Specifically, results from both studies suggested that a shared function of supervision and consultation seems to be knowledge consolidation. The most frequently cited supervision intervention was teaching and instruction. The use of information-based teaching was a common element of consultation, and several other elements (e.g., Socratic questioning, sharing a personal experience, modeling) were used in the service of knowledge

\footnotetext{
2 Twenty-six different supervision interventions were reported in the model of clinical supervision put forth by Milne and colleagues (2008). The comparison between this model and the model of clinical consultation put forth in this dissertation includes the supervision interventions that occurred in upwards of $25 \%$ of articles included in the review. The following supervision interventions occurred in $25 \%$ or more of the articles: teaching and instruction (75\%), use of corrective feedback (63\%), live or video-based observation (42\%), goal-setting (38\%), question-andanswer methods (38\%), modeling (29\%), planning (25\%), and reinforcement/praise/support (25\%). The following interventions occurred in fewer than $25 \%$ of the articles reviewed: discussion (21\%), prompts $(21 \%)$, role-play (21\%), review/reflection (21\%), explanation (17\%), monitoring $(17 \%)$, summarizing (17\%), challenging (13\%), listening (8\%), problem-solving (8\%), rehearsal of skills (8\%), self-disclosure (13\%), and five other interventions that each appeared once among the articles reviewed.
} 
consolidation. Additionally, a shared function of supervision and consultation was providing feedback. Question-and-answer methods (e.g., information gathering and clarifying), modeling, planning or organization, and the use of praise were present in both contexts. The following supervision interventions were not identified as elements of consultation: goal-setting, role-play, and live or video-based observation of the learner; however, this may have been purely a function of context. Consultation provided in a different context or format (e.g., the parent study's other condition) could include this. Both models advanced the literature by proposing an empirically based depiction of a learning process that can be tested experimentally. Although the development of these models arose from similar aims, they were developed with very different methodologies. Milne and colleagues (2008) conducted a "best evidence synthesis" of the supervision literature, which involved coding and extracting quantitative and qualitative information from the extant literature (24 studies). The extracted information was then analyzed to construct a model. In contrast, the author of this dissertation analyzed transcribed audio recorded consultation calls from a realist lens to identify the primary elements of consultation in a sample of clinicians learning to deliver CPT.

Given these different methodological approaches, there were some key differences in the language that was used and the way the findings were presented. First, the model of supervision was simultaneously more comprehensive, yet more general, while the model of consultation was simultaneously more specific, yet more limited in scope. Second, Milne and colleagues (2008) reported 28 outcomes of supervision, while this dissertation identified proficient delivery of the therapy as 
the sole outcome of interest. Relatedly, Milne and colleagues (2008) conceptualized the outcomes of supervision as mechanisms (e.g., changes in supervisees' attitudes, motivation, self-awareness, or skills). By contrast, the dissertation examined only one outcome of interest (proficiency), and the processes that helped the learner achieve that proficiency were conceptualized as mechanisms. Third, Milne and colleagues (2008) identified 35 contextual variables from across 24 studies, which were then categorized into five themes. In the present study, which was based on data collected in a single context, 6 contextual factors were identified. Finally, an important difference was the overarching context in which the models were created. The studies used to derive the supervision model were primarily from the learning disabilities field and residential settings, and the trainees were from a range of groups. By comparison, the consultation model was derived from a sample of independently licensed mental health clinicians who were all learning to deliver CPT in outpatient settings.

Although the model put forth by Milne and colleagues (2008) served as a useful departure point for the present study, several important limitations precluded a more in-depth and nuanced comparison between the models of supervision and consultation. Specifically, the results presented by Milne and colleagues (2008) lacked precise and detailed descriptions of the supervision interventions, a dynamic explanation of how the elements of supervision may be connected, and an explicit theory of how supervision is posited to produce change. Specifically, this model did not present a detailed theory of how a learner is thought to progress through the various interventions of supervision to achieve the 
identified outcomes of supervision. Because the model of supervision is not dynamic or mechanistic in nature, it was often difficult to discern the researchers' explanation of how supervision works. By contrast, the greatest contributions of this dissertation are its detailed descriptions of each element of consultation, which are replete with examples extracted from consultation calls, the proposed explanation of how the various elements of consultation are connected, and the hypothesized mechanisms through which consultation is posited to lead to learning. The explicit articulation of pathways and flow is an important contribution of the present study.

In summary, this dissertation had a similar aim to the project undertaken by Milne and colleagues (2008). Results from both studies included the identification of key contextual factors that are thought to facilitate and hinder learning. Knowledge consolidation was identified as a primary function of both supervision and consultation. The models of supervision and consultation both identified primary elements, and included teaching, providing feedback, question-and-answer methods, and praise. Unlike the model of supervision, the model of consultation included neither goal-setting nor role-play as a primary element. Although these projects had similar aims, they employed different methodologies. Milne and colleagues conducted a "best evidence synthesis," while the author of this dissertation analyzed the content of consultation calls from a realist perspective. This led to significant differences in the nature of information gleaned from the studies and the language used to describe the studies' findings. For example, the model of supervision was simultaneously more comprehensive, yet more general, while the model of consultation was simultaneously more specific, yet more limited in scope. 
Additionally, the supervision model presented numerous outcomes of supervision, while the model of consultation included only one. Finally, the models were constructed from different samples. Importantly, the model of consultation addressed some of the limitations of the model of supervision. Specifically, it included detailed descriptions of each element of consultation, and how the elements may be connected, as well as hypothesized mechanisms through which consultation is posited to lead to learning.

\section{Objective 3}

This study's final aim was to discuss whether Kolb's theory of experiential learning applies to consultation. Models of supervision and consultation are theoretical explanations of how learning is thought to occur in specific contexts. Kolb's model of experiential learning is an attempt to explain the individual's learning process, and has been used to explain how supervision leads to learning in several disciplines. Learning is defined as "the process whereby knowledge is created through the transformation of experience. Knowledge results from the combination of grasping and transforming experience" (Kolb, 1984, p.41). Experiential learning is a particular type of learning, and it has been defined as "learning through reflection on doing" (Felicia, 2011, p. 1003). Kolb posits that effective learning occurs when the individual passes through a cycle that includes the following four stages: experiencing, reflecting, conceptualizing, and experimenting. This model emphasizes the critical role of active learning in knowledge and skill acquisition. Learning is a cyclical process that involves the transformation of experience and occurs in these four stages. In their model of 
supervision, Milne and colleagues (2008) proposed that the learning that occurs in supervision could be explained according to Kolb's (1984) theory of experiential learning, and that the activities of supervision could be categorized according to these four stages.

Given the utility of Kolb's theory in understanding how supervision leads to learning, the helpfulness of this framework was explored as an explanatory framework for the primary findings of this dissertation. Accordingly, one of the objectives of this dissertation was to consider whether Kolb's theory of experiential learning applied to the learning that occurs during consultation, and whether the activities of consultation could be categorized according to these same stages. The first finding was that consultation on its own did not fit with Kolb's model of experiential learning. This is because a key component of the theory is "Experiencing". However, the combination of delivering the therapy and participating in consultation was consistent with Kolb's theory. Essentially, "Experiencing" and "Experimenting" seemed to occur when clinicians delivered the therapy and "Reflecting" and "Conceptualizing" seemed to occur when clinicians participated in consultation. In other words, it is posited that consultation alone does not lead to proficiency. Rather, it seems to be the combination of delivering the therapy and participating in consultation.

The first stage, "Experiencing," seemed to occur when clinicians delivered the therapy. Delivering the therapy gave clinicians the opportunity to participate in concrete learning, and to develop their procedural knowledge. Delivering the therapy offered an encounter with a new experience. At this stage, the clinicians' 
understanding was often challenged. The mismatch between the clinicians' expectations of what would occur in session and what actually occurred prompted them to reflect, which seemed to move them toward the next stage of the cycle.

The second stage, "Reflecting," is thought to be essential to the success of consultation. This hypothesized mechanism seems to be an indispensible component of the learning. The consultants, who engaged in various behaviours to encourage the clinicians to reflect, supported it. By asking the clinicians if they had questions (e.g., "So, you came up with some questions?” [Transcript 27, p. 1]), inviting them to reflect or participate (e.g., "[Name X], [Name Y], do you guys have anything to add there, or any other thoughts?" [Transcript 29, p. 7]), and engaging them in Socratic questioning (e.g., "What else are you all hearing, [Name X], and [Name Y]? I hear a lot of control, but I also hear some other things." [Transcript 28, p. 25]), the consultants fostered a culture of reflection, which appeared to facilitate learning. Additionally, clinicians' capacity for reflection likely contributed to the outcome of consultation. Clinicians who were more reflective by nature, and who came prepared with cases to discuss and questions to ask (e.g., "What happens with his account when he doesn't remember the major part of it?" [Transcript 19, p. 18]), likely derived greater benefit from the consultation process compared to clinicians who came unprepared and asked few questions.

The third stage, "Conceptualization," is thought to be the outcome of "Reflection". In the Conceptualization phase, that which was reflected on was assimilated and distilled into abstract concepts. In other words, at this stage, clinicians either formed new ideas based on their reflections, or modified their 
existing ideas according to new information. Socratic questioning is an element of consultation that likely plays an important role in moving a clinician from Reflection toward Conceptualization. Elements of consultation such as information-based teaching, sharing a personal experience, and case conceptualization are thought to be integral at this stage. Elements of consultation such as Giving Advice (e.g., "I wouldn't focus on that on this sheet...I would definitely want to focus on the part she is not getting before I would focus on alternative thoughts" [Transcript 13, p. 2]) and Modeling (e.g., "I would say, ‘There’s really one assignment in here where I really want you to kind of write out things. It may be very difficult, but there is some reason why we want you to write these things down."' [Transcript 14, p. 28]), which are directive and action-oriented, seemed to help move the clinician toward the next stage of the cycle.

The fourth stage, "Experimenting," refers to planning what to implement and trying out what has been learned. This occurred when clinicians implemented the advice they were given, or took the steps in session they realized were appropriate to take with their client. The degree to which clinicians developed proficiency likely largely depended on their ability to incorporate the learning and advice they received in consultation and apply it in session. Clinicians who were best able to accurately appraise what they needed help with, and translate the consultant's help into actionable steps to take during therapy may have been most likely to succeed at this stage. 


\section{Discussion}

Research has supported the integral role of clinical consultation as a strategy for helping clinicians become proficient in the delivery of evidence-based therapies (e.g., Beidas et al., 2012; Edmunds et al., 2013a). Despite the widely acknowledged importance of consultation, little is known about the essential elements of consultation (e.g., Edmunds et al., 2013a; Nadeem et al., 2013a.), and there is no existing theory positing how it works. Researchers have identified the pressing need to deepen our understanding of consultation by describing what consultation typically entails (e.g., Herschell et al., 2010; Rakovshik \& McManus, 2010), and identifying the mechanisms through which it works (e.g., Nadeem et al., 2013a). To address this gap in the literature, this study involved developing an explanatory model of clinical consultation and discussing it in light of the existing literature.

The study had two primary aims. The first was to describe what goes on in clinical consultation. This involved describing the content and function of the primary elements of consultation, developing a theory explaining how consultation is posited to work, and proposing $\mathrm{CMO}$ configurations from a realist lens that reflect the processes of consultation. The second aim was to compare the derived model of consultation to other models of learning that posit how clinicians become proficient in delivering therapies.

Results revealed that several contextual factors seem to influence the consultation process. Some of the contextual factors facilitated clinician learning, while others hindered it. Knowledge consolidation and case conceptualization were identified as consultation's overarching functions. To create an environment in 
which learning can occur, consultants must organize consultation calls effectively. Several elements of consultation were categorized as Organization. Other elements of consultation were categorized as follows: Asking for Help, Directive Instruction, Non-Directive Instruction, and Provision of Feedback. These elements seemed to play an integral role in facilitating knowledge consolidation and case conceptualization. Reflection and connectedness were hypothesized to be the primary mechanisms of consultation. Following the identification of the primary elements of consultation, a comprehensive theory explaining how consultation works was proposed, and three CMO configurations were presented.

Due to their shared facets, the derived model of consultation was compared with the model of supervision put forth by Milne and colleagues (2008). Knowledge consolidation was identified as being a primary function of both supervision and consultation, and the importance of the expert providing feedback to the learner was highlighted. Finally, the findings were discussed in relation to Kolb's theory of experiential learning. This suggested that it is the combination of delivering a new therapy and participating in consultation that leads to proficiency. Additionally, the centrality of reflection in the learning process was highlighted, in particular in relation to case conceptualization.

Overall, this study's findings are consistent with the burgeoning literature on consultation. In particular, technology's dual capacities to either facilitate or hinder learning has been identified elsewhere (e.g., Beidas et al., 2013). When asked about her experiences in consultation, one therapist reported that the computerized check-ins kept her accountable and connected to the group. However, another 
therapist reported feeling like computerized consultation undermined feelings of connectedness. Specifically, he felt a sense of disconnect with the other group members that he attributed to the lack of meeting in person. Others also commented on the potential for technological malfunctions to undermine connectedness. Frequently, therapists who were not technologically savvy reported that their struggle to use the technology created a barrier to cohesion. Ultimately, the effect of technology on consultation seemed to depend mostly on the quality of the technology and the way in which its use was experienced by the clinician.

Although the current study represents the most comprehensive effort to delineate the elements of consultation, previous researchers have presented preliminary findings on what are believed to be effective consultation techniques. For example, the use of teaching and instruction during consultation has been noted elsewhere (e.g., Edmunds et al., 2013a), and one group suggested continued training (i.e., knowledge consolidation) is a primary function of consultation (Nadeem et al., 2013a). Additionally, the importance of discussing real cases has been well documented (e.g., Accurso, Taylor, \& Garland, 2011; Edmunds et al., 2013a) and one group of researchers has posited that case support, which includes case conceptualization, is a primary function of consultation (Nadeem et al., 2013a). This study's identification of knowledge consolidation and case conceptualization as principal functions of consultation is consistent with the results of previous studies that have highlighted their importance.

Additionally, the importance of feedback has been highlighted repeatedly in the literature (e.g., Edmunds et al., 2013a; Miller et al., 2004; Ivers et al., 2012; 
Haynes, Smith, Dysthe, \& Ludvigsen, 2012). Therapists who were interviewed about what was most helpful during consultation expressed that they valued the receipt of consultant feedback, and in some cases wanted more of it (Beidas et al., 2013). This bolsters this study's finding that the provision of feedback was essential, and likely contributed directly to clinicians' development of proficiency. It is noteworthy that the majority of direct feedback provided to clinicians in our sample was delivered as praise. Although identified as an important element of consultation, correction was one of the elements used the least frequently. Perhaps because the clinicians who received consultation were already licensed, they were experienced in remaining adherent to a therapy protocol. Accordingly, it is likely that they generally conducted treatment in a manner that was consistent with the protocol and sought advice as needed. Perhaps in a sample of novice clinicians, supervisors would use correction more frequently.

In addition to efforts to identify the elements of consultation, researchers have tried to elucidate its mechanisms (e.g., Beidas et al., 2013; Edmunds et al., 2013b). One set of findings has led researchers to propose that connectedness may be the most important ingredient in successful consultation (Beidas et el., 2013b). Consistent with this research, which presents connectedness as a hypothesized mechanism of consultation, participants in the study conducted by Beidas and colleagues (2013b) described various activities of consultation and how they could help generate feelings of connectedness. Therapists expressed a preference for activities they viewed as authentic, and authenticity was identified as being a facilitator of connectedness. In particular, therapists valued discussion about real 
cases. Discussion of authentic case material occurred regularly during the consultation calls that informed the development of the proposed model.

Additionally, consultants' sharing of personal experiences created opportunities for authenticity and connectedness. Therefore, failing to bring cases to consultation may have undermined this process, since it limited the opportunities for connection through discussion of authentic case material. Interestingly, although role-play has been recommended for use as a consultation strategy, participants in the study conducted by Beidas and colleagues (2013b) expressed a dislike for role-play, explaining that the simulated nature of the exercised threatened both authenticity and connectedness. Interestingly, role-play was not used during consultation in the sample of clinicians who were learning to deliver CPT. In sum, the importance ascribed to connectedness in the aforementioned study (Beidas et al., 2013b) is supported by the findings of this dissertation, which suggested that connectedness is likely a mechanism through which consultation works.

In the model of consultation developed in this study, reflection has been put forth as a hypothesized mechanism of consultation. Although it has not been articulated previously in the literature, results from several studies support the possibility that reflection may be a mechanism of consultation. Specifically, one group of researchers highlighted self-evaluation (i.e., reflection through guided discovery) as an important technique used during consultation (Edmunds et al., 2013a). In another study, clinicians identified hearing each other's cases and challenges as an important part of consultation, explaining that it provided the opportunity for them to recognize and reveal their own challenges (Beidas et al., 
2013b). Although it was not described in these terms, clinicians' descriptions of what they found helpful during consultation permitted the possibility that reflection may be a mechanism through which learning occurred. Additionally, Kolb’s (1984) writing has emphasized the centrality of reflection in learning. His experiential learning cycle has been used as an explanatory framework for the outcomes of supervision (Milne et al., 2008), and has been proposed as a potentially applicable framework to the learning that occurs in consultation as well (Edmunds et al., 2013b). Other researchers have described reflection as the "engine" that drives therapist ongoing skill development more generally (Bennett-Levy, Thwaites, Chaddock, \& Davis, 2009). Although the explicit articulation of reflection as a hypothesized mechanism of consultation is a novel proposition, it is in accordance with the findings of previous research.

\section{Recommendations for Clinical Practice}

Results of this study, coupled with the emerging literature on clinical consultation, can be used to inform clinical consultation for clinicians learning to deliver CPT and other evidence-based therapies for PTSD. Based on the findings presented above, several recommendations can be made. First, policymakers should not underestimate the integral role technology can play in facilitating the dissemination and implementation of CPT by creating virtual learning communities. To ensure technology facilitates learning, rather than hinders it, organizations should invest in good quality technology. Doing so ought to limit the number of technological disruptions. Ideally, good quality technology will yield a strong Internet connection, resulting in few interruptions or delays on the call, and 
contribute to a high sound quality that will enable group members to hear one another clearly. Additionally, to ensure no one experiences technology as a barrier, organizations should subscribe to a secure service that is user-friendly. It is essential that all group members understand how to log on to the conferencing service and know how to utilize its primary functions at the outset of consultation.

Second, policymakers and consultants ought to emphasize the importance of regular attendance and punctuality. Consultants should do whatever they can to minimize disruptions on the calls due to clinician lateness. Consultants should model punctuality by ensuring they are on time for each call. When clinicians are late, consultants may consider using behavioural strategies to ensure lateness is not reinforced. If clinicians are late repeatedly, consultants should address this explicitly - either by speaking with the clinician directly, or by reminding the group about the importance of punctuality. This will help ensure each clinician receives the highest possible dose of consultation, and minimize the threat to feelings of connectedness that result from clinicians joining the call late.

Third, consultants should encourage clinicians to come prepared for consultation with questions and case material to discuss. When clinicians repeatedly fail to participate actively in consultation, consultants should take the time to identify their barriers, and support clinicians to become more active during consultation.

Fourth, consultants should take the time they need to organize the group at the beginning of the call. This includes identifying who is on the call and setting an agenda before clinicians begin asking questions and delving into case material. This 
may be particularly important when consultation is conducted virtually, since participants cannot see one another. Creating and maintaining structure on the call seems to be essential in ensuring adequate time is devoted to all the clinicians' needs.

Fifth, consultants should be cognizant of knowledge consolidation as a principal function of consultation. Accordingly, consultants should willingly devote time during consultation to review CPT's underlying theory, terminology, and session content. Particularly toward the beginning of consultation, consultants should review and elaborate on the content presented at the foundational workshop. To ensure clinicians have an accurate understanding of the therapy, consultants can test clinicians' knowledge of the protocol informally (e.g., by "quizzing" clinicians on the call), or provide them with a written quiz about the content of CPT. Given that their knowledge of the protocol will have a direct impact on the delivery of the therapy, consultants should ensure all the clinicians in their group have an accurate and thorough understanding of CPT. To support knowledge consolidation, consultants should normalize clinicians' feelings that they have lingering questions about CPT after the standard foundational workshop, and encourage them to ask questions about the protocol.

Sixth, as with knowledge consolidation, consultants should recognize that case conceptualization is a principal function of consultation. Accordingly, they should devote considerable time during consultation to case conceptualization, ensuring that the clinicians in their group have strong case conceptualizations, which are updated routinely as they gather more information about their clients. 
Additionally, consultants should keep track of which cases are presented on each call, ensure all cases are presented regularly, and explicitly discuss changes to the conceptualizations. Before giving advice, consultants should ensure clinicians have strong case conceptualizations. In recognizing the bidirectional relationship between knowledge consolidation and case conceptualization, consultants can illustrate general principles by using the specifics of the clinicians' cases. Helping clinicians develop their case conceptualization skills by making explicit the connection to CPT's underlying theory should help them develop proficiency in CPT. Finally, being mindful of the primary functions of consultation (i.e., knowledge consolidation and case conceptualization) can help consultants appraise the usefulness of the strategies they employ in consultation.

Seventh, the provision of feedback is essential, as it may contribute directly to proficiency. Although it can be difficult to offer adequate individualized feedback in a group setting, consultants should take the time to do so. Consultants ought to consider what each clinician is doing well and what he or she needs to improve. In addition to providing feedback during consultation, it is probably advantageous for clinicians to receive written feedback from someone who has reviewed their recorded session with a client.

Finally, the identification of hypothesized mechanisms through which consultation functions should serve to guide consultants' strategy, irrespective of which elements of consultation they employ. Specifically, consultants should focus on fostering connectedness and encouraging reflection. Consultants can increase feelings of connectedness between themselves and their group members by sharing 
their own experiences with the clinicians. They can create opportunities for connectedness among group members by encouraging clinicians to try and answer each other's questions. Consultants should be mindful of behaviours that undermine connectedness. For example, consultants should intervene if clinicians are absent or late repeatedly. To encourage reflection, consultants should use Socratic questioning whenever possible, and invite clinicians to reflect on and contribute to discussions about each other's cases. Doing so may be extremely helpful for clinician learning, since it encourages both connectedness and reflection. Relatedly, consultants should consider limiting their positioning of themselves as experts, particularly once consultation is well underway. Ideally, consultants should let clinicians offer their suggestions on other clinicians' cases first, and offer their own idea only if a clinician has not offered it already. If group members offer a variety of suggestions, consultants may want to use praise to highlight the advice they believe the clinician ought to follow.

\section{Limitations and Future Directions}

This study represents an important step toward the development of evidence-based guidelines for consultation in CPT. The use of observational coding conducted through a realist lens permitted the identification of consultation's primary functions and elements, as well as the development of a comprehensive theory and three CMO configurations positing how consultation works. Along with its merits, this study has important limitations that require discussion.

An important limitation of this study was the inability to assess the relationship between what occurred during consultation and clinicians' behaviour 
during sessions. Since there is a large body of research suggesting that consultation improves clinician fidelity to protocol (e.g., Beidas et al., 2012; Rakovshik et al., 2016), a positive relationship between consultation and proficiency was assumed in this study. Future studies should investigate the relationship between consultation and clinician learning. In particular, researchers should assess both clinician knowledge about the protocol and changes in clinician proficiency over time, and how each is related to what occurs during consultation.

This study's methodology resulted in a significant increase of information about the elements of consultation and processes through which it is posited to work. Given the novel nature of this project, there is little existing research with which to compare the study's findings. An important next step is to replicate this study using a similar methodology. This could be done by using the codebook developed for this study as a draft codebook to code consultation calls in another sample of clinicians learning CPT. If the model is confirmed, it would be advantageous to employ a similar methodology in a sample of clinicians learning to deliver a cognitive-behavioural therapy other than CPT. Doing so may begin to reveal to what extent the findings of this study can be generalized. Understanding the generalizability of findings has implications for whether evidence-based consultation guidelines need to be therapy-specific.

Since the results of realist evaluations are often theoretical explanatory models (Wong et al., 2014), subsequent steps involve examining the results through empirical investigation. An important next step to this study is to examine which elements, or combination of elements, are most effective. This could be done 
through a dismantling study comparing the entirety of consultation to elements that support its two principal functions - knowledge consolidation and case conceptualization. In the knowledge consolidation condition, consultants would be urged to focus on reviewing CPT's underlying theory, key terms, and session content. Consultants and group members might offer advice on how to proceed, but detailed case conceptualization focused explicitly on connecting the theory underlying CPT to specific cases would be proscribed. In the case conceptualization condition, consultants would be urged to focus on discussing the specifics of clinicians' cases, and formulating what is contributing to clients' problems. If clinicians posed questions related to CPT's underlying theory or session content, consultants would instruct clinicians to review their workshop materials and treatment manual. Researchers should assess both clinicians' knowledge about the protocol and clinicians' skill in delivering the protocol, and how each of these changes over time. Results of this study could help researchers identify the optimal focus of consultation, which could lead to more efficient training.

Given the study's aim of identifying the primary elements of consultation, the frequency with which each element was used and whether this varied over the course of consultation was not recorded. Perhaps earlier consultation sessions ought to include more didactics, while middle sessions ought to include a greater emphasis on case conceptualization, and later sessions ought to include more directive instruction, such as giving advice and modeling. Future studies should investigate whether certain strategies are used more frequently and are more valuable at different points in consultation. To assess this, researchers could code 
the presence of various elements of consultation, and evaluate whether the frequency with which they are used varies according to the phase of consultation. Identifying the relative importance of various elements of consultation by phase of consultation may help consultants tailor the focus of consultation to optimize clinician learning.

Finally, future research should examine the purported mechanisms of consultation. To do so, researchers could conduct semi-structured interviews with clinicians who participated in consultation focused on their experiences in consultation and what they found most helpful. To prevent bias, questions posed near the beginning of the interview could be more general (e.g., "What parts of consultation do you think most directly contributed to your learning?"), while those posed closer to the end of the interview could be more specific (e.g., "What role did reflection play in your learning"?"). In addition to using qualitative methods to test the hypothesized mechanisms of consultation, quantitative methods could be used. As a first step, researchers ought to develop valid measures to assess the constructs of "connectedness" and "reflection". Using valid and reliable assessment tools, researchers could identify the presence of these hypothesized mechanisms during consultation calls and assess their role as a mediator or moderator of clinician learning.

\section{Summary and Conclusions}

The successful dissemination and implementation of CPT requires postworkshop consultation (Monson et al., under review). Research in the area of clinical consultation has been impeded by poor conceptualization of its elements 
and mechanisms, as well as by the absence of a theory explaining how consultation works. This study advances the literature by providing a detailed description of the content and function of the primary elements of clinical consultation, and proposing a theory of how consultation contributes to the proficient delivery of CPT.

Based on the results of this study, recommendations for how to optimize CPT consultation have been made. Specifically, organizations ought to invest in high quality technology to minimize technological disruptions during consultation. Consultants ought to encourage clinicians to arrive on time for consultation, and to come prepared with questions and case material to discuss. Consultants should focus on consultation's core functions of knowledge consolidation and case conceptualization, and mindfully employ other strategies to support these functions. Although the group context can make this challenging, consultants should aim to provide clinicians with ample feedback on their delivery of CPT. Importantly, consultants should be attuned to consultation as a process, and harness its hypothesized mechanisms - connectedness and reflection. In particular, consultants should provide clinicians with ample opportunities to reflect on each other's questions and cases, rather than jumping in and giving expert advice.

The model of consultation put forth in this study advances our understanding how consultation works. The identification of the primary elements and hypothesized mechanisms of consultation provides researchers with a solid foundation for investigating what makes for effective consultation. An important next step is to test the proposed theory, and to assess the relationship between the use of various elements of consultation, the development of clinician proficiency, 
and patient outcomes. The results of this study represent an important step toward the development of evidence-based guidelines in clinical consultation, which may contribute to the improvement of patient outcomes. 
Appendix A

Comparison of three post-workshop conditions

\begin{tabular}{|c|c|c|c|}
\hline & $\begin{array}{c}\text { No } \\
\text { Consultation/Fidelity } \\
\text { Monitoring alone }\end{array}$ & $\begin{array}{c}\text { Consultation As } \\
\text { Usual }\end{array}$ & $\begin{array}{c}\text { Tech Enhanced } \\
\text { Consultation }\end{array}$ \\
\hline $\begin{array}{l}\text { Access to handouts, } \\
\text { manuals, and other } \\
\text { support materials }\end{array}$ & Yes & Yes & Yes \\
\hline $\begin{array}{l}\text { Access to CPT fidelity } \\
\text { monitoring measure }\end{array}$ & Yes & Yes & Yes \\
\hline $\begin{array}{l}\text { Requirements/measures } \\
\text { for ensuring regular } \\
\text { clinician participation }\end{array}$ & $\begin{array}{l}\text { Study team monitors } \\
\text { to ensure regular } \\
\text { session uploading }\end{array}$ & $\begin{array}{l}\text { Consultants } \\
\text { encourage } \\
\text { participation } \\
\text { through check- } \\
\text { ins at each } \\
\text { consultation }\end{array}$ & $\begin{array}{l}\text { Consultants } \\
\text { track and } \\
\text { ensure that all } \\
\text { clinicians } \\
\text { present audio } \\
\text { regularly }\end{array}$ \\
\hline $\begin{array}{l}\text { Weekly Consultation } \\
\text { Calls }\end{array}$ & No & Yes & Yes \\
\hline $\begin{array}{l}\text { Discussion of Case } \\
\text { Material }\end{array}$ & No & Yes & Yes \\
\hline Role Plays & No & $\begin{array}{l}\text { Allowed, not } \\
\text { required }\end{array}$ & $\begin{array}{l}\text { Encouraged } \\
\text { (e.g., in } \\
\text { response to } \\
\text { session } \\
\text { segments) }\end{array}$ \\
\hline $\begin{array}{l}\text { Discussion of Fidelity } \\
\text { Ratings }\end{array}$ & No & No & Yes \\
\hline $\begin{array}{l}\text { Uploading/Review of } \\
\text { stuck point logs and } \\
\text { worksheets }\end{array}$ & No & No & Yes \\
\hline Review of session audio & No & No & $\begin{array}{l}\text { Yes-required in } \\
\text { each session }\end{array}$ \\
\hline
\end{tabular}


Appendix B

\begin{tabular}{|c|c|c|c|c|}
\hline \multicolumn{5}{|c|}{ Contextual Factors } \\
\hline Name of code & Definition & When to use & When not to use & Example \\
\hline Clinician joins late & $\begin{array}{l}\text {-Clinician is not present } \\
\text { at beginning of call, but } \\
\text { joins call once } \\
\text { discussion is underway }\end{array}$ & $\begin{array}{l}\text {-When clinician joins } \\
\text { more than a few } \\
\text { minutes into the call, or } \\
\text { misses considerable } \\
\text { content }\end{array}$ & $\begin{array}{l}\text {-When clinician is not } \\
\text { present at very } \\
\text { beginning of call, but } \\
\text { joins while the } \\
\text { consultant is taking } \\
\text { attendance, or has not } \\
\text { missed considerable } \\
\text { content by the time he } \\
\text { or she joins the call } \\
\end{array}$ & $\begin{array}{l}\text { E: Oh, it looks like } \\
\text { we've got } \\
\text { somebody. Hi, who } \\
\text { joined? }\end{array}$ \\
\hline $\begin{array}{l}\text { Clinician does not have } \\
\text { content to discuss }\end{array}$ & $\begin{array}{l}\text {-Clinician is present on } \\
\text { call, but does not have } \\
\text { any questions to ask, or } \\
\text { case material to present }\end{array}$ & $\begin{array}{l}\text {-Clinician has not yet } \\
\text { begun working with a } \\
\text { client, or has not seen a } \\
\text { client since the last } \\
\text { consultation call, and } \\
\text { therefore does not have } \\
\text { case material to discuss } \\
\text {-Clinician does not have } \\
\text { any questions }\end{array}$ & $\begin{array}{l}\text {-Clinician asks } \\
\text { questions, or brings } \\
\text { case material to discuss }\end{array}$ & $\begin{array}{l}\text { C: I have nothing } \\
\text { new this week } \\
\text { because I'm seeing } \\
\text { my clients every } \\
\text { second week and } \\
\text { they are both on } \\
\text { the week coming } \\
\text { up. I talked about } \\
\text { my clients last } \\
\text { week and I don't } \\
\text { have anything new. }\end{array}$ \\
\hline
\end{tabular}




\begin{tabular}{|l|l|l|l|l|}
\hline $\begin{array}{l}\text { Only one clinician on } \\
\text { call }\end{array}$ & $\begin{array}{l}\text {-When multiple } \\
\text { clinicians are absent } \\
\text { from the call, and only } \\
\text { one clinician is present }\end{array}$ & $\begin{array}{l}\text {-If call begins with one } \\
\text { clinician on, and } \\
\text { another joins mid-way } \\
\text { through the call so the } \\
\text { majority of the call has } \\
\text { more than one clinician }\end{array}$ & $\begin{array}{l}\text { E: It looks like you } \\
\text { are the only one on } \\
\text { the call. }\end{array}$ \\
\hline $\begin{array}{l}\text { Technological } \\
\text { disruptions }\end{array}$ & $\begin{array}{l}\text {-Technological } \\
\text { malfunction during a } \\
\text { call }\end{array}$ & $\begin{array}{l}\text {-Common examples } \\
\text { include: difficulty } \\
\text { logging into the server, } \\
\text { difficulty hearing, call } \\
\text { cutting in and out, call is } \\
\text { dropped repeatedly, } \\
\text { and portions of of audio } \\
\text { recording is inaudible }\end{array}$ & $\begin{array}{l}\text { E: That sounds fine. } \\
\text { (Transcriptionist's } \\
\text { note: Inaudible, } \\
\text { recording blip). } \\
\text { (Several } \\
\text { respondents advise } \\
\text { that they are having } \\
\text { difficulty hearing } \\
\text { the expert). }\end{array}$ \\
\hline
\end{tabular}


Appendix C

\begin{tabular}{|c|c|c|c|c|}
\hline \multicolumn{5}{|c|}{ Primary Elements of Consultation } \\
\hline Draft Name of code & Definition & When to use & When not to use & Examples \\
\hline Agenda setting & $\begin{array}{l}\text {-Organizing time } \\
\text {-Deciding what the call } \\
\text { will be used for } \\
\text {-Discussion of what } \\
\text { clinicians would like help } \\
\text { with or have questions } \\
\text { about }\end{array}$ & $\begin{array}{l}\text {-Code should only be } \\
\text { applied to expert } \\
\text { speech } \\
\text {-Captures a list of items } \\
\text { to be discussed on the } \\
\text { call } \\
\text {-Agenda is set near the } \\
\text { beginning of the call }\end{array}$ & $\begin{array}{l}\text {-When expert does not } \\
\text { delineate topics for call } \\
\text { in advance, but segues } \\
\text { from one topic to the } \\
\text { next ("Setting/ } \\
\text { maintaining structure" } \\
\text { would likely apply) }\end{array}$ & $\begin{array}{l}\text { Example \#1 } \\
\text { E: So, we should set } \\
\text { an agenda for } \\
\text { today's } \\
\text { conversation. If } \\
\text { each one of you } \\
\text { could give me a } \\
\text { brief couple of } \\
\text { minutes...how } \\
\text { many patients you } \\
\text { have got and what } \\
\text { sessions you are in } \\
\text { with that patient, } \\
\text { what is going on } \\
\text { with the PCL and } \\
\text { any questions that } \\
\text { you might have that } \\
\text { you would like us to } \\
\text { address. Just make } \\
\text { it brief. We'll get } \\
\text { into the weeds once } \\
\text { we get started, just } \\
\text { so I have a sense of } \\
\text { what we need to } \\
\text { talk about today. Of } \\
\text { course, let me know }\end{array}$ \\
\hline
\end{tabular}




\begin{tabular}{|c|c|c|c|c|}
\hline & & & & $\begin{array}{l}\text { if there is anything } \\
\text { urgent. } \\
\text { Example \#2 } \\
\text { E: Then, X, we'll let } \\
\text { you volunteer first. } \\
\text { And then, we'll go } \\
\text { from there and try } \\
\text { to touch base with } \\
\text { everyone. Go ahead, } \\
\text { X. }\end{array}$ \\
\hline $\begin{array}{l}\text { Asking if clinicians } \\
\text { have questions }\end{array}$ & $\begin{array}{l}\text {-Asking if anyone has } \\
\text { questions } \\
\text {-The solicitation of } \\
\text { questions can be very } \\
\text { specific or more general }\end{array}$ & $\begin{array}{l}\text {-Code should only be } \\
\text { applied to expert } \\
\text { speech }\end{array}$ & & $\begin{array}{l}\text { Example \#1 } \\
\text { E: Any other issues } \\
\text { come up for you in } \\
\text { session one or two } \\
\text { that would be } \\
\text { helpful for me to } \\
\text { review? } \\
\text { Example \#2 } \\
\text { E: Do you have any } \\
\text { specific questions? }\end{array}$ \\
\hline $\begin{array}{l}\text { Asking for help/ } \\
\text { advice/ guidance/ } \\
\text { feedback }\end{array}$ & $\begin{array}{l}\text {-Asking expert if she has } \\
\text { advice or suggestions on } \\
\text { how to approach a } \\
\text { situation or deal with a } \\
\text { particular issue } \\
\text {-Seeking guidance in a }\end{array}$ & $\begin{array}{l}\text {-Code should only be } \\
\text { applied to clinician } \\
\text { speech } \\
\text {-Question pertains to } \\
\text { case formulation, } \\
\text { conceptual issues, or }\end{array}$ & $\begin{array}{l}\text {-When seeking facts or } \\
\text { information ("Posing a } \\
\text { fact-based question" } \\
\text { would likely apply) }\end{array}$ & $\begin{array}{l}\text { Example \#1 } \\
\text { C: The other issue is } \\
\text { assistance with } \\
\text { dealing with people } \\
\text { with very high } \\
\text { anger and a new } \\
\text { gentleman I have, }\end{array}$ \\
\hline
\end{tabular}




\begin{tabular}{|c|c|c|c|c|}
\hline & $\begin{array}{l}\text { direct or indirect manner } \\
\text {-Making one's needs or } \\
\text { questions apparent, } \\
\text { obvious, or known } \\
\text {-Think about as being } \\
\text { parallel to the sort of } \\
\text { information captured by } \\
\text { the "experiential-based } \\
\text { teaching" code }\end{array}$ & $\begin{array}{l}\text { what action to take with } \\
\text { a client } \\
\text {-Includes asking for } \\
\text { feedback on whether } \\
\text { the consultant would } \\
\text { have handled } \\
\text { something the same } \\
\text { way }\end{array}$ & & $\begin{array}{l}\text { I've had him for two } \\
\text { or three sessions } \\
\text { now and he just flat } \\
\text { out says, "Look, I } \\
\text { am black and white. } \\
\text { There is no grey } \\
\text { about anything." } \\
\text { Example \#2 } \\
\text { C: And, do I get him } \\
\text { to do that as his } \\
\text { homework again, or } \\
\text { would I get him to } \\
\text { do that right in } \\
\text { session? }\end{array}$ \\
\hline Attendance & $\begin{array}{l}\text {-Taking role call } \\
\text {-Identifying who is on } \\
\text { the call } \\
\text {-A statement about who } \\
\text { is on the call, who is } \\
\text { going to join the call, or } \\
\text { who is absent but is } \\
\text { expected to be on the call }\end{array}$ & $\begin{array}{l}\text {-Code can be applied to } \\
\text { either expert or } \\
\text { clinician speech, or a } \\
\text { combination }\end{array}$ & $\begin{array}{l}\text {-If a clinician joins later } \\
\text { in the call this should be } \\
\text { captured with the } \\
\text { contextual factor } \\
\text { "Clinician joins late" }\end{array}$ & $\begin{array}{l}\text { Example \#1 } \\
\text { E: I do have all your } \\
\text { names, so let me } \\
\text { just check to see } \\
\text { who is all on the } \\
\text { call. Uh, I see on left } \\
\text { hand side it looks } \\
\text { like X, are you here? } \\
\text { C: Yes I am, hello. } \\
\text { E: And X? } \\
\text { C: Hi. }\end{array}$ \\
\hline
\end{tabular}




\begin{tabular}{|c|c|c|c|}
\hline & & & $\begin{array}{l}\text { E: Hi. And X? } \\
\text { C: Yes. Good } \\
\text { morning. } \\
\text { E: Okay, and that's } \\
\text { all that's showing. } \\
\text { I'm not seeing X or } \\
\text { X. Are they on and } \\
\text { I'm just not seeing } \\
\text { them? } \\
\text { C: No I haven't } \\
\text { heard them. } \\
\text { Example \#2 } \\
\text { E: Who is on so far? } \\
\text { C1: Um, well I am X. } \\
\text { C2: Hi, X. It's X. }\end{array}$ \\
\hline Case conceptualization & $\begin{array}{l}\text {-Providing background } \\
\text { information about a } \\
\text { client and his/ her } \\
\text { trauma } \\
\text {-Describing the course of } \\
\text { treatment }\end{array}$ & $\begin{array}{l}\text {-Code can be applied to } \\
\text { either expert or } \\
\text { clinician speech, a } \\
\text { conversation between } \\
\text { an expert and a } \\
\text { clinician, or several } \\
\text { group members }\end{array}$ & $\begin{array}{l}\text { Example \#1 } \\
\text { C: I saw the other } \\
\text { patient with the } \\
\text { motorcycle accident } \\
\text { today and we did } \\
\text { session six and we } \\
\text { spent a large } \\
\text { portion of the }\end{array}$ \\
\hline
\end{tabular}




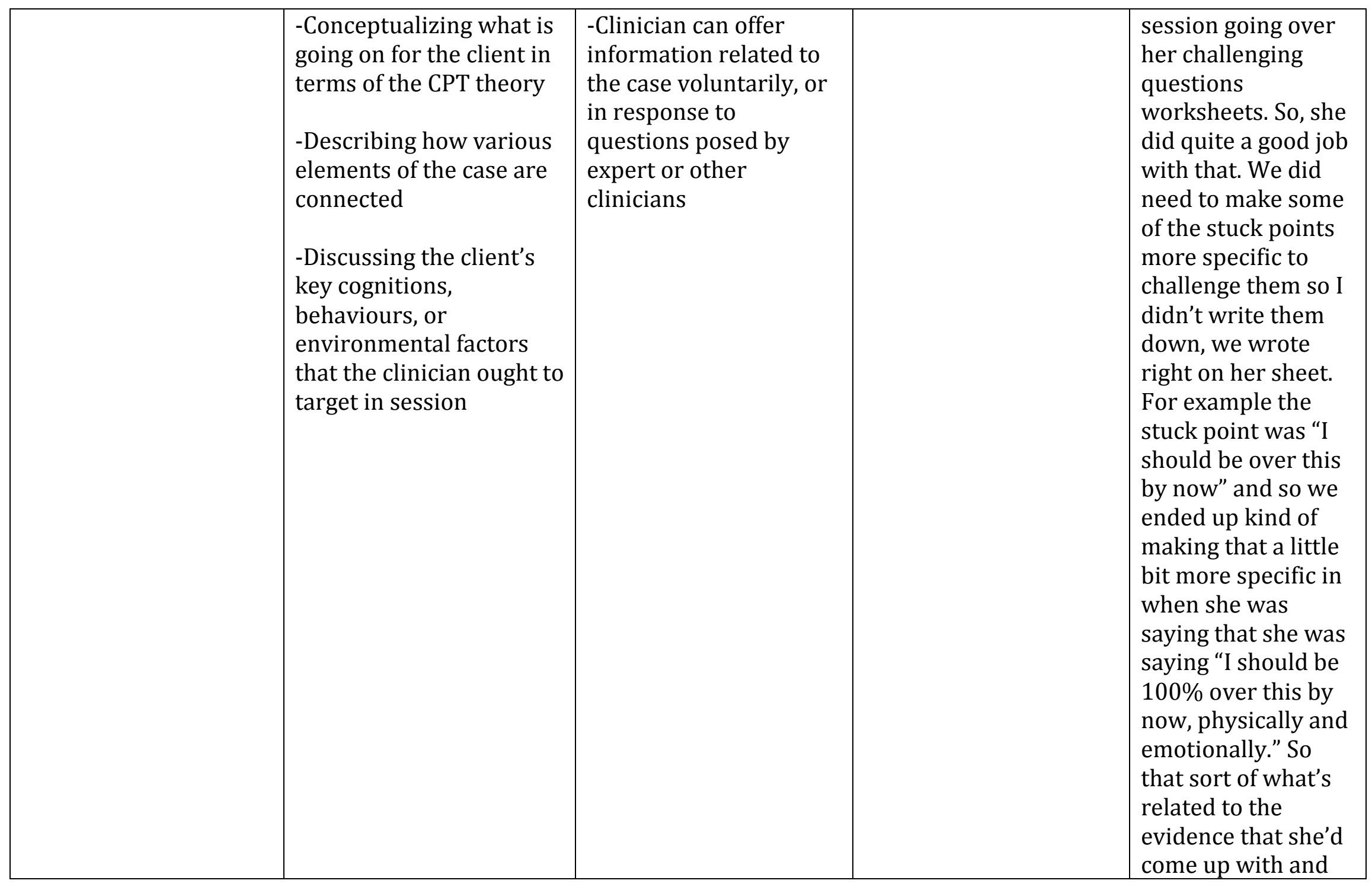




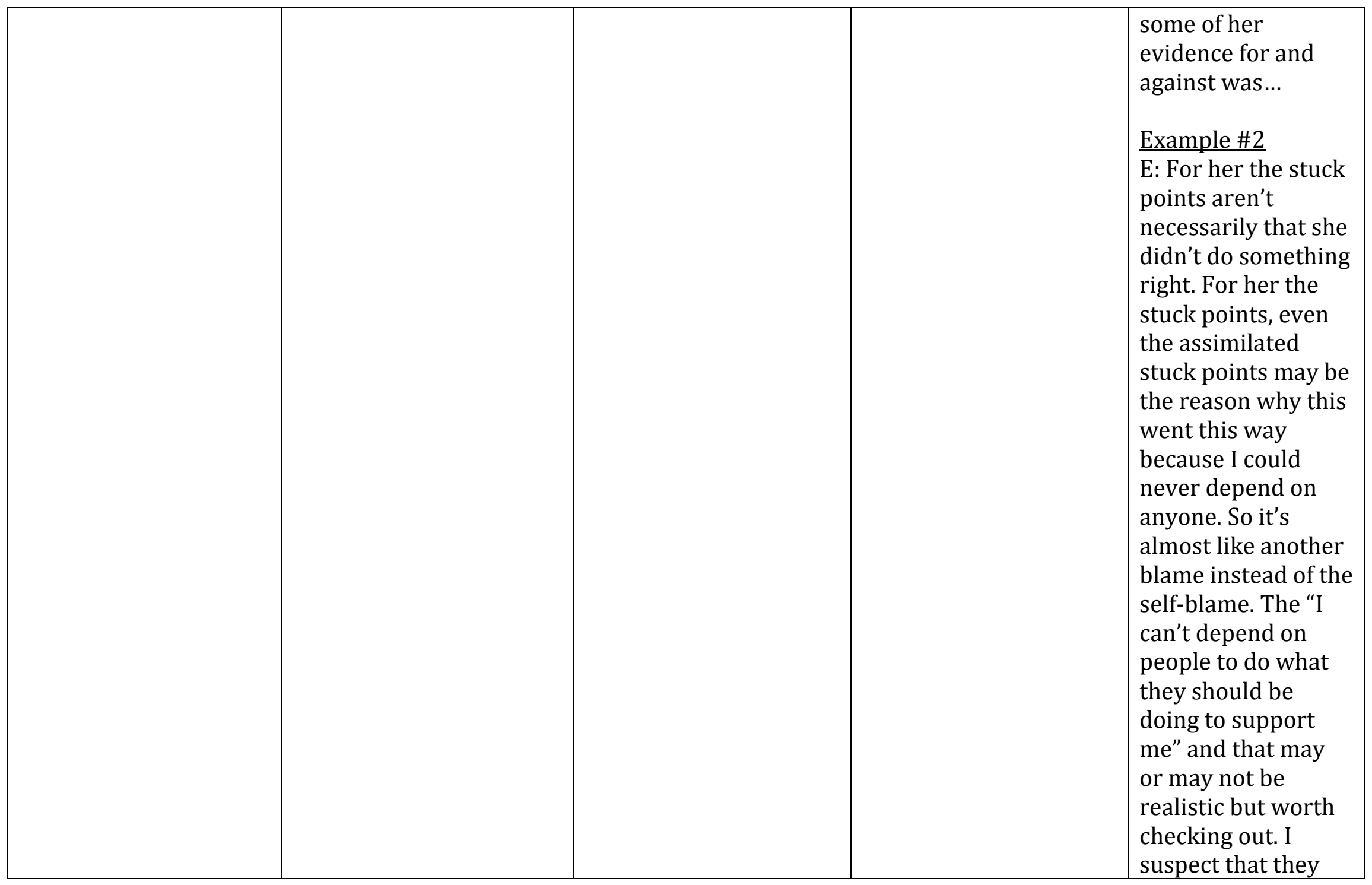




\begin{tabular}{|c|c|c|c|c|}
\hline & & & & $\begin{array}{l}\text { probably are people } \\
\text { around that can } \\
\text { support her but } \\
\text { she's so confident } \\
\text { that she may } \\
\text { preclude them from } \\
\text { doing that. }\end{array}$ \\
\hline Clarification & $\begin{array}{l}\text {-Seeking to confirm } \\
\text { understanding; to } \\
\text { elucidate, remove } \\
\text { ambiguity or doubt; to } \\
\text { clear of confusion or } \\
\text { uncertainty }\end{array}$ & $\begin{array}{l}\text {-Code can be applied to } \\
\text { either expert or } \\
\text { clinician speech, a } \\
\text { conversation between } \\
\text { an expert and a } \\
\text { clinician, or several } \\
\text { group members } \\
\text {-Consultants seek } \\
\text { clarification about a } \\
\text { clinician's actions or } \\
\text { understanding } \\
\text {-Clinicians seek } \\
\text { clarification by sharing } \\
\text { their understanding } \\
\text { and asking the } \\
\text { consultant to clarify or } \\
\text { correct them as needed } \\
\text {-When it is apparent } \\
\text { that the intent is to } \\
\text { clarify }\end{array}$ & $\begin{array}{l}\text {-Essence of the } \\
\text { statement should not be } \\
\text { about providing a } \\
\text { rationale or explanation }\end{array}$ & $\begin{array}{l}\text { Example \#1 } \\
\text { E: So I just want to } \\
\text { clarify, you started } \\
\text { giving a PCL, you } \\
\text { started saying } \\
\text { session three but } \\
\text { you actually did } \\
\text { three and fourth. } \\
\text { C: Yeah I did three } \\
\text { and four. So, } \\
\text { um...yeah okay but I } \\
\text { also gave you the } \\
\text { PCL for session two. } \\
\text { Example \#2 } \\
\text { C: So, you're asking } \\
\text { about the scores for } \\
\text { the tools? } \\
\text { E: The PTSD } \\
\text { symptoms, yes. } \\
\text { How are the PTSD }\end{array}$ \\
\hline
\end{tabular}




\begin{tabular}{|c|c|c|c|c|}
\hline & & & & symptoms? \\
\hline Correction & $\begin{array}{l}\text {-Highlighting something } \\
\text { that the clinician did that } \\
\text { was incorrect } \\
\text {-Pointing out an error (in } \\
\text { understanding, } \\
\text { conceptualization, } \\
\text { execution etc.) }\end{array}$ & $\begin{array}{l}\text {-Code should only be } \\
\text { applied to expert } \\
\text { speech }\end{array}$ & $\begin{array}{l}\text {-An error in the client's } \\
\text { thinking would likely be } \\
\text { captured under "Case } \\
\text { presentation/ } \\
\text { formulation" }\end{array}$ & $\begin{array}{l}\text { Example \#1 } \\
\text { E: There was one } \\
\text { stuck point that you } \\
\text { mentioned, “It's } \\
\text { ideal to have } \\
\text { several contingency } \\
\text { plans." I'm not sure } \\
\text { if that's a stuck } \\
\text { point because it } \\
\text { probably is ideal... } \\
\text { Example \#2 } \\
\text { C: X, I'm only seeing } \\
\text { my clients every } \\
\text { second week or so. } \\
\text { E: Ooh, that's not } \\
\text { good. That } \\
\text { encourages a lot of } \\
\text { avoidance. We } \\
\text { actually don't have } \\
\text { any research that } \\
\text { that's a good idea. } \\
\text { Is there a way you } \\
\text { could possibly get } \\
\text { this person either in } \\
\text { to see you every } \\
\text { week or if they } \\
\text { literally can't come }\end{array}$ \\
\hline
\end{tabular}




\begin{tabular}{|c|c|c|c|}
\hline & & & $\begin{array}{l}\text { in because of } \\
\text { money, do the } \\
\text { second week by } \\
\text { phone? }\end{array}$ \\
\hline Discussion about study & $\begin{array}{l}\text {-Discussion about study } \\
\text { logistics, policies etc. }\end{array}$ & $\begin{array}{l}\text {-Code can be applied to } \\
\text { either expert or } \\
\text { clinician speech, a } \\
\text { conversation between } \\
\text { an expert and a } \\
\text { clinician, or several } \\
\text { group members } \\
\text {-Common examples } \\
\text { include clinicians } \\
\text { posing questions } \\
\text { related to study } \\
\text { logistics, and expert } \\
\text { describing how } \\
\text { processes differ } \\
\text { between clinical } \\
\text { practice and the study }\end{array}$ & $\begin{array}{l}\text { Example \#1 } \\
\text { E: I am not sure } \\
\text { what the decision } \\
\text { has been for this } \\
\text { study...So what I } \\
\text { might do is e-mail X } \\
\text { because he is the } \\
\text { one taking care of } \\
\text { all the logistics and } \\
\text { can probably let } \\
\text { you know. } \\
\text { Example \#2 } \\
\text { C: I have started the } \\
\text { study with one } \\
\text { client who has a } \\
\text { PTSD diagnosis. } \\
\text { And we met two } \\
\text { times to prepare } \\
\text { him for the study. } \\
\text { We are meeting for } \\
\text { the third time today } \\
\text { where were are } \\
\text { starting the consent } \\
\text { forms and into } \\
\text { steps. }\end{array}$ \\
\hline
\end{tabular}




\begin{tabular}{|c|c|c|c|c|}
\hline Encouragement & $\begin{array}{l}\text {-Feeling or expectation } \\
\text { that something specific is } \\
\text { likely to occur } \\
\text {-To give confidence or } \\
\text { hope about the therapy } \\
\text { going forward, or about } \\
\text { the progress they can } \\
\text { expect to make learning } \\
\text { the treatment }\end{array}$ & $\begin{array}{l}\text {-Code should only be } \\
\text { applied to expert } \\
\text { speech } \\
\text {-Future-oriented }\end{array}$ & $\begin{array}{l}\text {-When discussion is } \\
\text { focused on something } \\
\text { that was well-done, } \\
\text { "Praise" would likely } \\
\text { apply }\end{array}$ & $\begin{array}{l}\text { Example \#1 } \\
\text { E: It's kind of one of } \\
\text { those things where } \\
\text { it's daunting when } \\
\text { you start and it } \\
\text { becomes very } \\
\text { different once you } \\
\text { know those } \\
\text { components. } \\
\text { Example \#2 } \\
\text { I definitely think X } \\
\text { that you've got a } \\
\text { good understanding } \\
\text { and the fact that } \\
\text { we're having this } \\
\text { kind of discussion } \\
\text { at this point, this is } \\
\text { just coming up with } \\
\text { additional ideas but } \\
\text { I do get the sense } \\
\text { and I think with } \\
\text { more sessions like } \\
\text { you said, all the } \\
\text { nuts and bolts will } \\
\text { become more } \\
\text { second nature. }\end{array}$ \\
\hline $\begin{array}{l}\text { Experiential-based } \\
\text { teaching }\end{array}$ & $\begin{array}{l}\text {-Subjective or personal } \\
\text { knowledge gained in the } \\
\text { role of clinician, expert, }\end{array}$ & $\begin{array}{l}\text {-Code should only be } \\
\text { applied to expert } \\
\text { speech }\end{array}$ & $\begin{array}{l}\text {-When it includes } \\
\text { explicit instructions of } \\
\text { what a clinician should }\end{array}$ & $\begin{array}{l}\text { Example \#1 } \\
\text { E: I had a client } \\
\text { yesterday in my }\end{array}$ \\
\hline
\end{tabular}




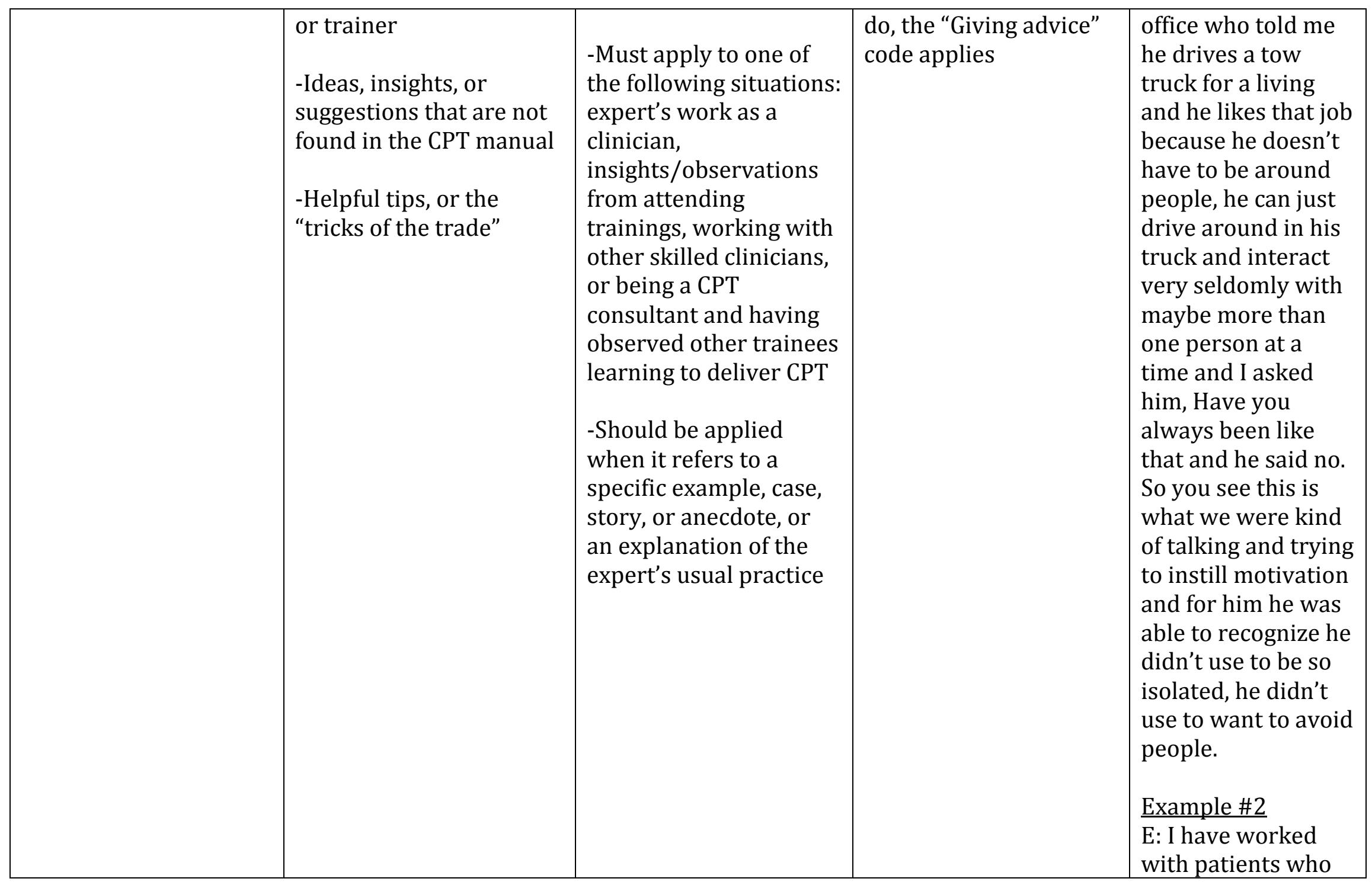




\begin{tabular}{|c|c|c|c|}
\hline & & & $\begin{array}{l}\text { clearly understand } \\
\text { that it is not their } \\
\text { fault. And yet, the } \\
\text { avoidance for these } \\
\text { kinds of reasons } \\
\text { that you have listed, } \\
\text { in terms of stuck } \\
\text { points...the } \\
\text { avoidance is } \\
\text { powerful enough } \\
\text { that they don't get } \\
\text { an opportunity to } \\
\text { really digest the } \\
\text { experience to the } \\
\text { extent that they } \\
\text { don't have to be } \\
\text { experiencing the } \\
\text { hyperarousal that } \\
\text { just never gets a } \\
\text { chance to settle } \\
\text { down. }\end{array}$ \\
\hline Giving advice & $\begin{array}{l}\text {-The imparting of } \\
\text { instructions, } \\
\text { recommendations, } \\
\text { guidance, or suggestions } \\
\text { for how to proceed } \\
\text {-The giving of advice } \\
\text { typically results in steps } \\
\text { or actions for the }\end{array}$ & $\begin{array}{l}\text {-Code should only be } \\
\text { applied to expert } \\
\text { speech } \\
\text {-Can be specific to the } \\
\text { case being discussed, or } \\
\text { can be more general } \\
\text { advice about how to } \\
\text { handle particular types }\end{array}$ & $\begin{array}{l}\text { Example \#1 } \\
\text { E: One of the things } \\
\text { that I might } \\
\text { recommend is } \\
\text { setting an agenda } \\
\text { with her at the } \\
\text { beginning... } \\
\text { Example \#2 }\end{array}$ \\
\hline
\end{tabular}




\begin{tabular}{|c|c|c|c|c|}
\hline & clinician to take & $\begin{array}{l}\text { of clients, situations, } \\
\text { issues etc. }\end{array}$ & & $\begin{array}{l}\text { E: Definitely you're } \\
\text { going to stay } \\
\text { trauma focused and } \\
\text { work on those stuck } \\
\text { points that are } \\
\text { related to kind of } \\
\text { how he's } \\
\text { remembering that } \\
\text { trauma before you } \\
\text { reach into those } \\
\text { over- } \\
\text { accommodated } \\
\text { ones which it } \\
\text { sounds like he's } \\
\text { kind of told you to } \\
\text { do the over- } \\
\text { accommodated } \\
\text { ones but we really } \\
\text { want to make sure } \\
\text { that we're spending } \\
\text { enough time on the } \\
\text { memory, on the } \\
\text { past, the } \\
\text { assimilated ones. }\end{array}$ \\
\hline $\begin{array}{l}\text { Information-based } \\
\text { teaching }\end{array}$ & $\begin{array}{l}\text {-Imparting information } \\
\text { related to PTSD (e.g., } \\
\text { symptoms, PCL scores) } \\
\text { or CPT (e.g., elements of } \\
\text { the protocol, information } \\
\text { about the protocol, or the }\end{array}$ & $\begin{array}{l}\text {-Code should only be } \\
\text { applied to expert } \\
\text { speech } \\
\text {-The content that is } \\
\text { being communicated is }\end{array}$ & $\begin{array}{l}\text {-If the expert draws on } \\
\text { her own experience to } \\
\text { illustrate a point, the } \\
\text { "Experiential-based } \\
\text { teaching" code should } \\
\text { be used }\end{array}$ & $\begin{array}{l}\text { Example \#1 } \\
\text { E: Just to kind of } \\
\text { review session one, } \\
\text { the clients aren't } \\
\text { really getting to say } \\
\text { much of anything. If }\end{array}$ \\
\hline
\end{tabular}




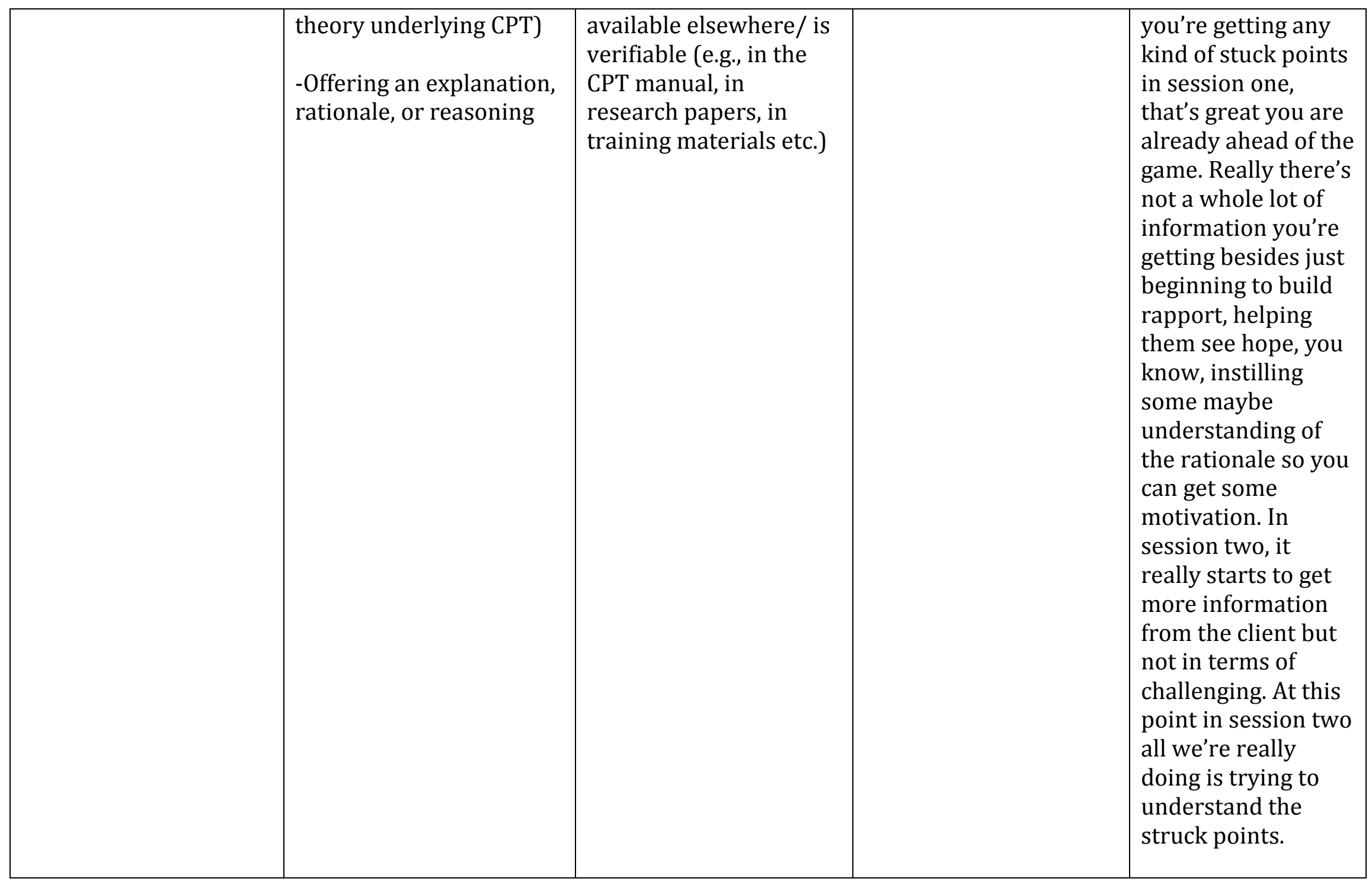




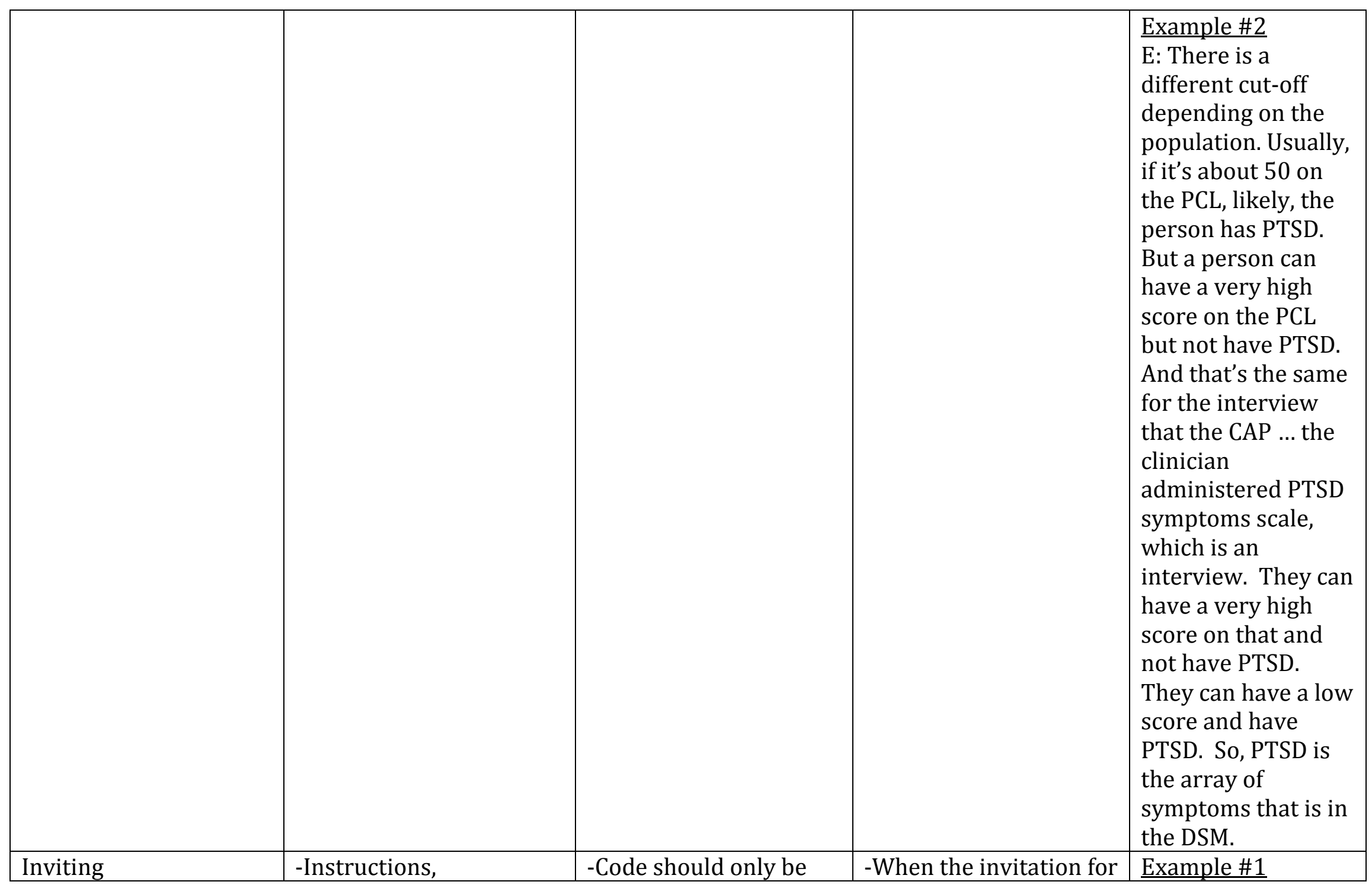




\begin{tabular}{|c|c|c|c|c|}
\hline participation/reflection & $\begin{array}{l}\text { recommendations, } \\
\text { guidance, or suggestions } \\
\text { for how to proceed } \\
\text {-Being directive } \\
\text {-Advice from an expert } \\
\text { would typically result in } \\
\text { steps or actions for the } \\
\text { clinician to take } \\
\text {-This could be specific to } \\
\text { the case, or more general } \\
\text { advice about how to } \\
\text { handle particular types } \\
\text { of clients, situations, } \\
\text { issues, etc. }\end{array}$ & $\begin{array}{l}\text { applied to expert } \\
\text { speech }\end{array}$ & $\begin{array}{l}\text { participation is } \\
\text { connected to setting an } \\
\text { agenda the "Agenda } \\
\text { setting" code is applied } \\
\text {-When the invitation for } \\
\text { participation pertains } \\
\text { to figuring out which } \\
\text { clinicians are going to } \\
\text { present, then the } \\
\text { "Setting/ maintaining } \\
\text { structure" code is } \\
\text { applied } \\
\text {-When the invitation for } \\
\text { participation occurs in } \\
\text { the context of eliciting } \\
\text { more details about the } \\
\text { client/case, the "Case } \\
\text { presentation/ } \\
\text { formulation" code is } \\
\text { applied }\end{array}$ & $\begin{array}{l}\text { E: So that's just kind } \\
\text { of some thoughts } \\
\text { about that. What do } \\
\text { you all think? } \\
\text { Example \#2 } \\
\text { E: X, did you have } \\
\text { any thoughts that } \\
\text { you wanted to add } \\
\text { into this } \\
\text { conversation about } \\
\text { X's case? }\end{array}$ \\
\hline Modeling & $\begin{array}{l}\text {-Expert offers a clinician } \\
\text { a suggestion for what to } \\
\text { say suggestions for what } \\
\text { to say. } \\
\text {-Consultant does a } \\
\text { demonstration }\end{array}$ & $\begin{array}{l}\text {-Code should only be } \\
\text { applied to expert } \\
\text { speech } \\
\text {-Typically can be } \\
\text { identified with the use } \\
\text { of quotation marks }\end{array}$ & & $\begin{array}{l}\text { Example \#1 } \\
\text { E: What I might say } \\
\text { is something like, } \\
\text { "Okay so today } \\
\text { we're going to } \\
\text { review what you } \\
\text { did and I'm wanna } \\
\text { take a look at your }\end{array}$ \\
\hline
\end{tabular}




\begin{tabular}{|c|c|c|c|c|}
\hline & & $\begin{array}{l}\text {-Can be identified when } \\
\text { the expert gives the } \\
\text { clinician exact wording } \\
\text { to say to a client } \\
\text {-Since modeling is } \\
\text { usually in the service of } \\
\text { advice giving, when a } \\
\text { segment consists } \\
\text { mostly of wording for } \\
\text { the clinician to use, the } \\
\text { "Modeling" code is } \\
\text { applied }\end{array}$ & & $\begin{array}{l}\text { ABC sheet...I'll look } \\
\text { at your ABC sheet, } \\
\text { but I also need to } \\
\text { make sure we } \\
\text { spend some time, } \\
\text { probably the last } \\
\text { ten or fifteen } \\
\text { minutes talking } \\
\text { about your next } \\
\text { assignments." } \\
\text { Example \#2 } \\
\text { E: One of the ways } \\
\text { that I get people to } \\
\text { that is I'll say, "Well, } \\
\text { is this a change } \\
\text { from how you were } \\
\text { before?" }\end{array}$ \\
\hline $\begin{array}{l}\text { Posing a fact-based } \\
\text { question }\end{array}$ & $\begin{array}{l}\text {-Querying about } \\
\text { something defined, } \\
\text { particular, or fact-based } \\
\text {-Seeking specific } \\
\text { information }\end{array}$ & $\begin{array}{l}\text {-Code should only be } \\
\text { applied to clinician } \\
\text { speech } \\
\text {-Typically closed-ended } \\
\text {-Questions can be } \\
\text { posed in a direct or } \\
\text { indirect manner } \\
\text {-This code can be } \\
\text { thought of as being }\end{array}$ & $\begin{array}{l}\text {-Intention should not be } \\
\text { to clarify } \\
\text { ("Clarification" would } \\
\text { likely apply) } \\
\text {-If questions pertain to } \\
\text { study logistics, apply } \\
\text { "Discussion related to } \\
\text { the study" }\end{array}$ & $\begin{array}{l}\text { Example \#1 } \\
\text { C: I'm just } \\
\text { wondering if you } \\
\text { can remind me } \\
\text { what a stuck point } \\
\text { would sound like if } \\
\text { it was an } \\
\text { assimilated one } \\
\text { versus an } \\
\text { accommodated one } \\
\text { Example \#2 }\end{array}$ \\
\hline
\end{tabular}




\begin{tabular}{|c|c|c|c|c|}
\hline & & $\begin{array}{l}\text { parallel to the } \\
\text { "information-based } \\
\text { teaching" code in that } \\
\text { the answers to these } \\
\text { questions could } \\
\text { probably be found in } \\
\text { the manual or other } \\
\text { CPT training materials }\end{array}$ & & $\begin{array}{l}\text { C: Uh, } \mathrm{X} \text {, a question. } \\
\text { If there is more } \\
\text { than one trauma, is } \\
\text { this where you } \\
\text { would say, If there } \\
\text { is other trauma } \\
\text { accounts go ahead } \\
\text { and write those out } \\
\text { as well and bring } \\
\text { them in? }\end{array}$ \\
\hline Praise & $\begin{array}{l}\text {-Expression of the } \\
\text { sentiment "good job" or } \\
\text { "nicely done" } \\
\text {-Identifying something } \\
\text { the clinician did well } \\
\text {-An expression of } \\
\text { approval or admiration }\end{array}$ & $\begin{array}{l}\text {-Code should only be } \\
\text { applied to expert } \\
\text { speech } \\
\text {-When the expert is } \\
\text { commenting on the } \\
\text { clinician's work } \\
\text {-The statement } \\
\text { simultaneously refers } \\
\text { to the clinician and the } \\
\text { client, or the statement } \\
\text { refers to the client's } \\
\text { progress that is } \\
\text { obviously connected to } \\
\text { the clinician's work }\end{array}$ & $\begin{array}{l}\text {-When the expert is } \\
\text { commenting on the } \\
\text { client's progress in } \\
\text { therapy }\end{array}$ & $\begin{array}{l}\text { Example \#1 } \\
\text { E: You guys have } \\
\text { got some really } \\
\text { impressive stuck } \\
\text { points I'm noticing } \\
\text { as I'm hearing } \\
\text { almost all the stuck } \\
\text { points that all three } \\
\text { of you brought up } \\
\text { and talked about } \\
\text { really sound well } \\
\text { worded. } \\
\text { Example \#2 } \\
\text { E: I think it sounds } \\
\text { like you're on the } \\
\text { right track. } \\
\text { Everything you've } \\
\text { said today makes }\end{array}$ \\
\hline
\end{tabular}




\begin{tabular}{|c|c|c|c|c|}
\hline & & & & $\begin{array}{l}\text { me feel like you } \\
\text { have a pretty good } \\
\text { sense about where } \\
\text { you're going with } \\
\text { this and the } \\
\text { protocol. }\end{array}$ \\
\hline Structure & $\begin{array}{l}\text {-Leading or directing the } \\
\text { flow of the call } \\
\text {-Segueing from one } \\
\text { topic/person to another } \\
\text {-Making sure one } \\
\text { clinician does not } \\
\text { monopolize the call } \\
\text { and/or that all issues } \\
\text { raised by clinicians get } \\
\text { addressed }\end{array}$ & $\begin{array}{l}\text {-Code should only be } \\
\text { applied to expert } \\
\text { speech } \\
\text {-Can include giving } \\
\text { instructions about how } \\
\text { to present cases } \\
\text {-Can occur at any point } \\
\text { in the call }\end{array}$ & $\begin{array}{l}\text {-When a list of items to } \\
\text { discuss on the call is } \\
\text { laid out at the } \\
\text { beginning of the call, } \\
\text { "Agenda setting" would } \\
\text { likely apply }\end{array}$ & $\begin{array}{l}\text { Example \#1 } \\
\text { E: I can address that } \\
\text { a little bit as well. } \\
\text { Thank you for that. } \\
\text { And that brings us } \\
\text { to X. } \\
\text { Example \#2 } \\
\text { E: Thank you so } \\
\text { much. Who would } \\
\text { like to go next? }\end{array}$ \\
\hline Socratic questioning & $\begin{array}{l}\text {-Technique used by } \\
\text { expert to encourage } \\
\text { clinician to reflect on a } \\
\text { concept, central idea, } \\
\text { application of the } \\
\text { protocol, or the way they } \\
\text { do things }\end{array}$ & $\begin{array}{l}\text {-Code can be applied to } \\
\text { either expert or } \\
\text { clinician speech, a } \\
\text { conversation between } \\
\text { an expert and a } \\
\text { clinician, or several } \\
\text { group members }\end{array}$ & $\begin{array}{l}\text {-Questions pertaining } \\
\text { to factual information } \\
\text { (e.g., "What session are } \\
\text { you on?" or "What is } \\
\text { your client's index } \\
\text { trauma"? do not meet } \\
\text { requirements for } \\
\text { "Socratic questioning" } \\
\text { code }\end{array}$ & $\begin{array}{l}\text { Example \#1 } \\
\text { E: And, so folks, } \\
\text { what do you think } \\
\text { the stuck point } \\
\text { might actually be...? } \\
\text { Example \#2 } \\
\text { E: X or X, what do } \\
\text { you think about } \\
\text { how to help } \\
\text { somebody? }\end{array}$ \\
\hline
\end{tabular}




\begin{tabular}{|c|c|c|c|}
\hline Validation & $\begin{array}{l}\text {-To confirm someone's } \\
\text { perspective, decisions, or } \\
\text { experience } \\
\text {-Expert takes the } \\
\text { perspective that what } \\
\text { clinician thought or did } \\
\text { makes sense or is ok }\end{array}$ & $\begin{array}{l}\text {-Code should only be } \\
\text { applied to expert } \\
\text { speech }\end{array}$ & $\begin{array}{l}\text { Example \#1 } \\
\text { E: I can understand } \\
\text { that because I know } \\
\text { we talked a lot } \\
\text { about trying to } \\
\text { format those stuck } \\
\text { points and that can } \\
\text { be time consuming } \\
\text { Example \#2 } \\
\text { E: I have had that } \\
\text { gentleman and I } \\
\text { had him Tuesday at } \\
2 \text { pm, so I can } \\
\text { identify with that } \\
\text { experience. }\end{array}$ \\
\hline
\end{tabular}




\section{References}

Accurso, E. C., Taylor, R. M., \& Garland, A. F. (2011). Evidence-based practices addressed in community-based children's mental health clinical supervision. Training and Education in Professional Psychology, 5(2), 88-96. doi:10.1037/a0023537

Alban, A., \& Frankel, S.A. (2007). Supervision vs. consultation: What you need to know. Clinical Lawyer. Retrieved from http://clinicallawyer.com/2007/07/ supervision-vs-consultation-what-you-need-to-know/

Andrzejewski, M. E., Kirby, K. C., Morral, A. R., \& Iguchi, M. Y. (2001). Technology transfer through performance management: The effects of graphical feedback and positive reinforcement on drug treatment counselors' behavior. Drug and alcohol dependence, 63(2), 179-86. doi: 10.1016/S0376-8716(00)00207-6

Alvesson, M., \& Skoldberg, K. (2000). Reflexive methodology: New vistas for qualitative research. Thousand Oaks, CA: SAGE Publications.

Beidas, R. S., Edmunds, J. E., Cannuscio, C. C., Gallagher, M., Downey, M. M., \& Kendall, P. C. (2013). Therapist perspectives on the effective elements of consultation following training. Administration and Policy in Mental Health and Mental Health Services, 40, 507-517. doi: 10.1007/s10488-013-0475-7

Beidas, R. S., Edmunds, J. E., Marcus, S. C., \& Kendall, P. C. (2012). Training and consultation to promote implementation of an empirically supported treatment: A randomized trial. Psychiatric Services, 63(7), 660-665. doi:10.1176/appi.ps. 201100401 
Beidas, R. S., \& Kendall, P. C. (2010). Training therapists in evidence-based practice: A critical review of studies from a systems-contextual perspective. Clinical Psychology, 17(1), 1-30. doi:10.1111/j.1468-2850.2009.01187.x

Bennett-Levy, J., McManus, F., Westling, B. E., \& Fennell, M. (2009). Acquiring and refining CBT skills and competencies: Which training methods are perceived to be most effective? Behavioural and Cognitive Psychotherapy, 37(5), 571-83. doi:10.1017/S1352465809990270

Bennett-Levy, J., Thwaites, R., Chaddock, A., \& Davis, M. (2009). Reflective practice in cognitive behavioural therapy: The engine of lifelong learning. In J. Steadmon and R. Dallos (Eds.), Reflective practice in psychotherapy and counselling (pp.115-135). New York, NY: McGraw-Hill.

Bernard, J.M., \& Goodyear, R.K. (1992). Fundamentals of clinical supervision. Needham Heights, MA: Allyn \& Bacon.

Bogo, M., \& Mcknight, K. (2006). Clinical supervision in social work: A review of the research literature. The Clinical Supervisor, 24, 49-67. doi:10.1300/J001v24n01

Braun, V., \& Clarke, V. (2006). Using thematic analysis in psychology. Qualitative research in psychology, 3(2), 77-101. doi: 10.1191/1478088706qp063oa

Brooks, C. T., Patterson, D. A., \& McKiernan, P. M. (2012). Group supervision attitudes: Supervisory practices fostering resistance to adoption of evidencebased practices. The Qualitative Report, 17(1), 191-199. Retrieved from http://www.nova.edu/ssss/ QR/QR17-1/brooks.pdf Caplan, G., \& Caplan, R. B. (1993). Mental health consultation and collaboration. San Francisco, CA: Jossey-Bass Publishers. 
Carpenter, K. M., Cheng, W. Y., Smith, J. L., Brooks, A. C., Amrhein, P. C., Wain, R. M., \& Nunes, E. V. (2012). “Old dogs” and new skills: How clinician characteristics relate to motivational interviewing skills before, during, and after training. Journal of Consulting and Clinical Psychology, 80(4), 560-73.

doi:10.1037/a0028362

Cohen, J. (1960). A coefficient for agreement for nominal scales. Education and Psychological Measurement, 20, 37-46. doi: 10.1177/001316446002000104

Cottrell, N.B., Wack, D.L., Sekerak, G.J., \& Rittle, R.M. (1968). Social facilitation of dominant responses by the presence of an audience and the mere presence of others. Journal of Personality and Social Psychology, 9, 245-250.

Dashiell, J.F. (1930). An experimental analysis of some group effects. Journal of Abnormal and Social Psychology, 25, 190-199.

Davy, J. (2002). Discursive reflections on a research agenda for clinical supervision. Psychology and Psychotherapy: Theory, Research and Practice, 75, 221-238. doi: $10.1348 / 147608302169661$

Dennis, A. R., \& Valacich, J. S. (1993). Computer brainstorms: More heads are better than one. Journal of Applied Psychology, 78, 531-537. doi: 10.1037/00219010.78 .4 .531

Dunn, C., Deroo, L., \& Rivara, F. P. (2001). The use of brief interventions adapted from motivational interviewing across behavioral domains: A systematic review. Addiction 96(12), 1725-1742. doi:10.1080/09652140120089481

Edmunds, J. M. (2013). An examination of active learning as an ingredient of 
consultation following training in cognitive-behavioral therapy for youth anxiety (Doctoral dissertation). Retrieved from https://ryerson.summon.serials solutions.com

Edmunds, J. M., Beidas, R. S., \& Kendall, P. C. (2013a). Dissemination and implementation of evidence-based practices: Training and consultation as implementation strategies. Clinical Psychology: Science and Practice, 20(2), 152165. doi: $10.1111 /$ cpsp.12031

Edmunds, J. M., Kendall, P. C., Ringle, V. A., Read, K. L., Broadman, D. M., Pimentel, S. S., \& Beidas, R. S. (2013b). An examination of behavioural rehearsal during consultation as a predictor of training outcomes. Administration and Policy in Mental Health and Mental Health Services, 40, 456-466. doi: 10.107/s10488013-0490-8

El-Tannir, A. A. (2002). The corporate university model for continuous learning, training and development. Education and Training, 44(2), 76-81. doi:10.1108/ 00400910210419973

Falender, C., \& Shafranske, E. (2004). Clinical supervision: A competence-based approach. Washington, DC: American Psychological Association.

Faugier B. (1994). Thin on the ground. Nursing Times 90, 64-67. doi: 10.1002/ cpp.297

Felicia, P. (2011). Handbook of research on improving learning and motivation through educational games. Ireland: Waterford Institute of Technology. Festinger, L. (1954). A theory of social comparison processes. Human Relations, 7, 117-140. 
Festinger, L., Back, K., Schachter, S., Kelley, H.H., \& Thibaut, J. (1950). Theory and experiment in social communication. Michigan, MI: Research Center for Dynamics, Institute for Social Research.

Fixsen, D. L., Naoom, S. F., Base, K. A., Friedman, R. M., \& Wallace, F. (2005) Implementation research: A synthesis of the literature. Tampa, FL: University of South Florida, Louis de la Parte. Florida Mental Health Institute, The National Implementation Research Network (FMHI Publication 231).

Flick, U. (2009) An introduction to qualitative research. London, England: SAGE Publications.

Foa, E. B., Hembree, E. A., \& Rothbaum, B. O. (2007). Prolonged exposure therapy for PTSD. New York, NY: Oxford University Press.

Foa, E.B., Keane, T.M., Friedman, M.J., \& Cohen, J.A. (Eds.). (2005). Effective treatments for PTSD: Practical guidelines from the International Society for Traumatic Stress Studies, $2^{\text {nd }}$ edition. New Yok, NY: Guilford Press.

Ford, J. D. (1979). Research on training counselors and clinicians. Review of Educational Research, 49(1), 87. doi:10.2307/1169928

Foulkes, S.H. (1964). Therapeutic group analysis. London: Allen and Unwin. Ganju, V. (2003). Implementation of evidence-based practices in state mental health systems: Implications for research and effectiveness studies. Schizophrenia Bulletin, 29(1), 125-31. Retrieved from http://www.ncbi.nlm.nih.gov/ pubmed/12908667

Godley, S. H., Garner, B. R., Smith, J. E., Meyers, R. J., \& Godley, M. D. (2011). A largescale dissemination and implementation model for evidence-based treatment 
and continuing care. Clinical Psychology, 18(1), 67-83. doi:10.1111/j.14682850.2011.01236.x

Gotham, H. J. (2004). Diffusion of mental health and substance abuse treatments: Development, dissemination, and implementation. Clinical Psychology: Science and Practice, 11(2), 161-176. doi:10.1093/clipsy/bph067

Gotham, H. J. (2006). Advancing the implementation of evidence-based practices into clinical practice: How do we get there from here? Professional Psychology: Research and Practice, 37(6), 606-613. doi:10.1037/0735-7028.37.6.606

Gregoire, T. K., Propp, J., \& Poertner, J. (1998). The supervisor's role in the transfer of training. Administration in Social Work, 22, 1-18.

Grimshaw, J., Shirran, L., Thomas, R., Mowatt, G., Fraser, C., Bero, L., ... O'Brien, M. (2001). Changing Provider Behavior: An Overview of Systematic Reviews of Interventions. Medical Care, 39(8), 112-1145. Retrieved from http://www.jstor.org/stable/3767642

Grol, R., \& Grimshaw, J. (1999). Evidence-based implementation of evidence-based medicine. The Joint Commission journal on quality improvement, 25(10), 503513. doi: 10.1016/S1070-3241(16)30464-3

Grol, R., \& Grimshaw, J. (2003). From best evidence to best practice: Effective implementation of change in patients' care. The Lancet, 362(9391), 1225-1230. doi: 10.1016/S0140-6736(03)1456-1

Gwet, K. L. (2014). Handbook of inter-rater reliability, $4^{\text {th }}$ edition. Gaithersburg, Maryland: Advanced Analytics, LLC.

Harkness, D., \& Poertner, J. (1989). Research and social work supervision: A 
conceptual review. Social Work, 34, 115-119.

Havnes, A., Smith, K., Dysthe, O., \& Ludvigsen, K. (2012). Formative assessment and feedback: Making learning visible. Studies in Educational Evaluation, 38(1), 2127. doi: 10.1016/j.sbspro.2014.12.1351

Herschell, A. D., Kolko, D. J., Baumann, B. L., \& Davis, A. C. (2010). The role of therapist training in the implementation of psychosocial treatments: A review and critique with recommendations. Clinical Psychology Review, 30(4), 448-466. doi: 10.1016/j.cpr.2010.02.005

Irvin, J. E., Bowers, C. A., Dunn, M. E., \& Wang, M. C. (1999). Efficacy of relapse prevention: A meta-analytic review. Journal of Consulting and Clinical Psychology, 67(4), 563-570. doi: 10.1037/0022-006X.67.4.563

Ivers, N., Jamtvedt, G., Flottorp, S., Young, J. M., Odgaard-Jensen, J., French, S. D., ... \& Oxman, A. D. (2012). Audit and feedback: effects on professional practice and healthcare outcomes. The Cochrane Library. doi: 10.1002/14651858. CD0 00259.pub3

Johnson, A.L., Crawford, M.T., Sherman, S.J., Rutchik, A.M., Hamilton, D.L., Ferreira, M.B., \& Petrocelli, J.V. (2006). A functional perspective on group memberships: Differential need fulfillment in group typology. Journal of Experimental Social Psychology, 42, 707-719. doi: 10.1016/j.jesp.2005.08.002

Joyce, B. R., \& Showers, B. (2002). Student achievement through staff development. Retrieved from: https://www.unrwa.org/sites/default/files/joyce_and_ showers_coaching_as_cpd.pdf 
Karlin, B. E., Ruzek, J.I., Chard, K. M., Efkhetari, A., Monson, C. M., Hembree, E. A., Resick, P. A., \& Foa, E. B. (2010). Dissemination of evidence-based psychological treatments for posttraumatic stress disorder in the veterans health administration. Journal of Traumatic Stress, 23(6), 663-673. doi:10. 1002/ jts. 20588

Kazdin, A. E. (2008). Evidence-based treatment and practice: New opportunities to bridge clinical research and practice, enhance the knowledge base, and improve patient care. The American Psychologist, 63(3), 146-159. doi:10.1037/0003066X.63.3.146

Kolb, D.A. (1984). Experiential learning. Upper Saddle River, NJ: Prentice-Hall.

Leshner, A.I. (1999). Science-based views of drug addiction and its treatment. The Journal of the American Medical Association, 282(14), 1314-1316. doi: 10.1001/jama.282.14.1314

Lewin, K. (1947). Frontiers in group dynamics: Concept, method and reality in social science; social equilibria and social change. Human Relations, 1(1), 5-41. doi: $10.1177 / 001872674700100103$

Martino, S., Ball, S. A., Nich, C., Frankforter, T. L., \& Carroll, K. M. (2008). Community program therapist adherence and competence in motivational enhancement therapy. Drug and Alcohol Dependence, 96, 37-48. doi:10.1016/j.drugalcdep.2008.01.020

Maruping, L. M., \& Agarwal, R. (2004). Managing team interpersonal processes through technology: A task-technology fit perspective. The Journal of Applied Psychology, 89(6), 975-90. doi:10.1037/0021-9010.89.6.975 
McGaghie, W.C., Siddall, V.J., Mazmanian, P.E., \& Myers, J. (2009). Lessons for continuing medical education from simulation research in undergraduate and graduate medical education: Effectiveness of continuing medical education. CHEST, 135(3_suppl), 62S-68S. doi: 10.1378/chest.08-2521

McHugh, R. K., \& Barlow, D. H. (2010). The dissemination and implementation of evidence-based psychological treatments. A review of current efforts. The American Psychologist, 65(2), 73-84. doi:10.1037/a0018121

Miller, W. R., Yahne, C. E., Moyers, T. B., Martinez, J., \& Pirritano, M. (2004). A randomized trial of methods to help clinicians learn motivational interviewing. Journal of Consulting and Clinical Psychology, 72(6), 1050-1062. doi:10.1037/0022-006X.72.6.1050

Milne, D, \& James, I. (2000). A systematic review of effective cognitive-behavioural supervision. The British Journal of Clinical Psycholog, 39, 111-127. doi: $10.1348 / 014466500163149$

Milne, D., Aylott, H., Fitzpatrick, H., \& Ellis, M.V. (2008). How does clinical supervision work? Using a "best evidence synthesis" approach to construct a basic model of supervision. The Clinical Supervisor, 27(2), 170-190. doi:10.1080/07325220802487915

Milne, D., \& Westerman, C. (2001). Evidence-based clinical supervision: Rationale and illustration. Clinical Psychology and Psychotherapy, 8, 444-457. doi:10.1002/cpp.297

Monson, C. M., Shields, N., Suvak, M., Lane, J. E., Shnaider, P., Landy, M. S. H,... 
Stirman, S. W. (under review). A randomized controlled effectiveness trial of training strategies in Cognitive Processing Therapy for posttraumatic stress disorder: Impact on patient outcomes.

Morse, J. M., Barrett, M., Mayan, M., Olson, K., \& Spiers, J. (2002). Verification strategies for establishing reliability and validity in qualitative research. International journal of qualitative methods, 1(2), 13-22. doi: $10.1177 / 160940690200100202$

Nadeem, E., Gleacher, A., \& Beidas, R. S. (2013a). Consultation as an implementation strategy for evidence-based practices across multiple contexts: Unpacking the black box. Administration and Policy in Mental Health and Mental Health Services Research, 40(6), 439-450. doi: 10.1007/s10488-013-0502-8

Nadeem, E., Gleacher, A., Pimental, S., Hill, L., McHugh, M., \& Hoagwood, K. (2013b). The role of consultation calls for clinic supervisors in supporting large-scale dissemination of evidence-based treatments for children. Administration and Policy in Mental Health Services Research. 40(6), 530-540. doi: 10.1007/s10488013-0491-7

Neuman, Y., Assaf, D., \& Cohen, Y. (2012). A novel methodology for identifying emerging themes in small group dynamics. Bulletin of the Menninger Clinic, 76(1), 53-68. doi:10.1521/bumc.2012.76.1.53

O’Hara-Devereaux, M., \& Johansen, R. (1994). Global work. San Francisco, CA: JosseyBass.

Pawson, R. (2013). The science of evaluation: A realist manifesto. doi: 10.4135/978 1473913820. 
Pawson, R., \& Tilley, N. (1997). An introduction to scientific realist evaluation. In E. Chelimsky \& W. R. Shadish (Eds.), Evaluation for the $21^{\text {st }}$ century: A handbook (pp. 405-418). Thousand Oaks, CA: SAGE Publications.

Pawson, R., \& Tilley, N. (2008). Realistic evaluation. London, England: SAGE Publications.

President's New Freedom Commission on Mental Health. (2003). Achieving the promise: Transforming mental health in American. Final report. Rockville, MD: DHHS Publication.

Proctor, E. K., Landsverk, J., Aarons, G., Chambers, D., Glisson, C., \& Mittman, B. (2009). Implementation research in mental health services: An emerging science with conceptual, methodological, and training challenges. Administration and Policy in Mental Health and Mental Health Services Research, 36(1), 24-34. doi:10.1007/s10488-008-0197-4

Rakovshik, S. G., \& McManus, F. (2010). Establishing evidence-based training in cognitive behavioral therapy: A review of current empirical findings and theoretical guidance. Clinical Psychology Review, 30(5), 496-516. doi: 10.1016/j.cpr.2010.03.004

Rakovshik, S. G., McManus, F., Vazquez-Montes, M., Muse, K., \& Ougrin, D. (2016). Is supervision necessary? Examining the effects of internet-based CBT training with and without supervision. Journal of Consulting and Clinical Psychology, 84(3), 191-199. doi: 10.1037/ccp0000079 
Raven, G. (2006). Methodological reflexivity: Towards evolving methodological frameworks through critical and reflexive deliberations. Environmental Education Research, 12(3-4), 559-569. doi: 10.1080/1350462060079923

Resick, P.A., Monson, C.M., \& Chard, K.M. (2014). Cognitive processing therapy: Veteran/military version. Washington, DC: Department of Veterans' Affairs.

Resick, P. A., \& Schnicke, M. K. (1992). Cognitive processing therapy for sexual assault victims. Journal of consulting and clinical psychology, 60(5), 748-756. doi: 10.1037//0022-006X.60.5.748

Saladaña, J. (2009). The coding manual for qualitative researchers. Los Angeles, CA: SAGE Publications.

Sambamurthy, V., Poole, M. S., \& Kelly, J. (1993). The effects of variations in GDSS capabilities on decision-making processes in groups. Small Group Research, 24, 523-546. doi: 10.1177/1046496493244006

Schoenwald, S. K., Sheidow, A. J., \& Chapman, J. E. (2009). Clinical supervision in treatment transport: Effects on adherence and outcomes. Journal of Consulting and Clinical Psychology, 77(3), 410-21. doi:10.1037/a0013788

Schoenwald, S. K., Sheidow, A. J., \& Letourneau, E. J. (2004). Toward effective quality assurance in evidence-based practice: Links between expert consultation, therapist fidelity, and child outcomes. Journal of Clinical Child and Adolescent Psychology, 33(1), 94-104. doi:10.1207/S15374424JCCP3301_10

Seta, J.J. (1982). The impact of comparison processes on coactors' task performance. Journal of Personality and Social Psychology, 42, 281-291. doi: 10.1177/ 0146167291175011 
Shaw, M.E. (1981). Group dynamics: The psychology of small group behavior, $3^{\text {rd }}$ edition. New York, NY: McGraw-Hill.

Shields, N. (2011, November). Closing the research loop: Implementing best practice treatment for PTSD in a network of operational stress injury clinics (Part B). Symposium presented at the 2nd Canadian Military Veteran Health Research Forum, Kingston, ON.

Sholomskas, D. E., Syracuse-Siewert, G., Rounsaville, B. J., Ball, S. A., Nuro, K. F., \& Carroll, K. M. (2005). We don't train in vain: A dissemination trial of three strategies of training clinicians in cognitive-behavioral therapy. Journal of Consulting and Clinical psychology, 73(1), 106-115. doi:10.1037/0022006X.73.1.106

Sobh, R., \& Perry, C. (2006). Research design and data analysis in realism research. European Journal of Marketing, 40(11/12), 1194-1209. doi:10.1108/03090 56061- 0702777

Strunk, D. R., Brotman, M. A., DeRubeis, R. J., \& Hollon, S. D. (2010). Therapist competence in cognitive therapy for depression: Predicting subsequent symptom change. Journal of Consulting and Clinical Psychology, 78(3), 429-437. doi:10.1037/a0019631

Townend, M., Iannetta, L., \& Freeston, M. H. (2002). Clinical supervision in practice: A survey of UK cognitive behavioural psychotherapists accredited by the BABCP. Behavioural and Cognitive Psychotherapy, 30, 485-500. doi:10.1017/ S1352465802004095 
Turner, J.C. (1987). Rediscovering the social group: A self-categorization theory. New York, NY: Basil Blackwell.

Triplett, N. (1898). The dynamogenic factors in pacemaking and competition. American Journal of Psychology, 9, 507-533. doi: 10.2307/1412188

Vandalay, A. (1996). Effectiveness of chip importation as means of truth conveyance and deflection in relationship dynamics. Journal of Popular Film and Television, 52, 600608. doi:10.3141/159265

Walters, S. T., Matson, S. A., Baer, J. S., \& Ziedonis, D. M. (2005). Effectiveness of workshop training for psychosocial addiction treatments: A systematic review. Journal of Substance Abuse Treatment, 29, 283-293. doi:10.1016/j.jsat.2005. 08.006

Weissman, M. M., Verdeli, H., Gameroff, M. J., Bledsoe, S. E., Betts, K., Mufson, L., Fitterling, H., \& Wickramaratne, P. (2006). National survey of psychotherapy training in psychiatry, psychology, and social work. Archives of General Psychiatry, 63, 925-934. doi:10.1001/archpsyc.63.8.925

Weisz, J. R., Ugueto, A. M., Herren, J., Afienko, S. R., \& Rutt, C. (2011). Kernels vs. ears, and other questions for a science of treatment dissemination. Clinical Psychology: Science and Practice, 18(1), 41-46. doi: 10.1007/s10488-013$0475-7$

Williams, C., \& Martinez, R. (2008). Increasing access to CBT: Stepped care and CBT self-help models in practice. Behavioural and Cognitive Psychotherapy, 36, 675683. doi:10.1017/S1352465808004864 
Wong, G., Greenhalgh, T., Westhorp, G., \& Pawson, R. (2014). Development of methodological guidance, publication standards and training materials for realist and meta-narrative reviews: the RAMESES (Realist And Meta-narrative Evidence Syntheses-Evolving Standards) project. Retrieved from https://www.ncbi.nlm.nih.gov/books/NBK260013/

Yalom, I.D. (1995). The theory and practice of group psychotherapy, $5^{\text {th }}$ edition. New York, NY: Basic Books Ltd.

Zajonc, R.B. (1965). Social facilitation. Science, 149, 269-274. Retrieved from: http://links.jstor.org/sici?sici=0036-8075\%282819650716\%293\%3A3681\% 3C269\%3ASF\%3E2.0.C0\%3B 